University of Texas at El Paso

\title{
DigitalCommons@UTEP
}

Open Access Theses \& Dissertations

2015-01-01

\section{Development of a Novel Hybrid Unified Viscoplastic Constitutive Model}

Luis Alejandro Varela Jimenez

University of Texas at El Paso, Lavarela@miners.utep.edu

Follow this and additional works at: https://digitalcommons.utep.edu/open_etd

Part of the Mechanical Engineering Commons

\section{Recommended Citation}

Varela Jimenez, Luis Alejandro, "Development of a Novel Hybrid Unified Viscoplastic Constitutive Model" (2015). Open Access Theses \& Dissertations. 1176.

https://digitalcommons.utep.edu/open_etd/1176

This is brought to you for free and open access by DigitalCommons@UTEP. It has been accepted for inclusion in Open Access Theses \& Dissertations by an authorized administrator of DigitalCommons@UTEP. For more information, please contact lweber@utep.edu. 


\title{
DEVELOPMENT OF A NOVEL HYBRID UNIFIED VISCOPLASTIC CONSTITUTIVE MODEL
}

\author{
LUIS ALEJANDRO VARELA JIMENEZ
}

Department of Mechanical Engineering

APPROVED:

Calvin M. Stewart, Ph.D., Chair

Yirong Lin, Ph.D.

David A. Roberson, Ph.D.

Charles Ambler, Ph.D.

Dean of the Graduate School 


\section{Copyright (C)}

by

Luis Alejandro Varela Jimenez

2015 
Dedicado a mi familia, a los de aquí y a los que desde arriba siguen aquí. 


\title{
DEVELOPMENT OF A NOVEL HYBRID UNIFIED VISCOPLASTIC CONSTITUTIVE MODEL
}

\author{
by \\ LUIS ALEJANDRO VARELA JIMENEZ, B.S.M.E.
}

\section{THESIS}

Presented to the Faculty of the Graduate School of The University of Texas at El Paso

in Partial Fulfillment

of the Requirements

for the Degree of

MASTER OF SCIENCE

Department of Mechanical Engineering THE UNIVERSITY OF TEXAS AT EL PASO

May 2015 


\section{ACKNOWLEDGEMENTS}

I would like to thank my advisor Dr. Calvin M. Stewart, whose guidance and advice was fundamental to complete my master degree. It has been an honor to work and learn from such a professional advisor. I want to state my gratitude to the Mechanical Engineering Department for all the opportunities that have been provided to me which have tremendously contributed to my professional career. Finally I would like to thank my family for being my inspiration and for all the provided support. 


\begin{abstract}
Gas turbines are now days used in power plants for power generation and for propulsion in the aerospace industry. In these applications gas turbines are exposed to severe temperature and pressure variations during operating cycles. These severe operating conditions exposed the turbine's components to multiple deformation mechanisms which degrade the material and eventually lead to failure of the components. Nickel based and austenitic super alloys are candidate material used for these applications due to its high strength and corrosion resistance at elevated temperatures. At such temperature levels, candidate materials exhibit a rate-dependent or viscoplastic behavior which difficult the prediction or description of the material response due to deformation mechanisms. Unified viscoplastic constitutive models are used to describe this viscoplastic behavior of materials. In the present work Miller and Walker unified viscoplastic models are presented, described and exercised to model the creep of Hastelloy X and the low cycle fatigue behavior of stainless steel 304. The numerical simulation results are compared to an extensive database of experimental data to fully validate the capabilities and limitations of the considered models. Material constant heuristic optimizer (MACHO) software is explained and used to determine both models material constants and ensure a systematic calculation of them. This software uses the simulated annealing algorithm to determine the optimal material constants values in a global surface, by comparing numerical simulations to an extensive database of experimental data. A quantitative analysis on the performance of both models is conducted to determine the most suitable model to predict material's behavior. Based on the two exercised classical viscoplastic models, a novel hybrid unified model is introduced, to accurately describe the inelastic behavior caused by creep and fatigue effects at high temperature. The presented
\end{abstract}


hybrid model consists on the combination of the best aspects of Miller and Walker model constitutive equations, with the addition of a damage rate equation which provides capabilities to describe damage evolution and life prediction for Hastelloy X and stainless steel 304. A detailed explanation on the meaning of each material constant is provided, along with its impact on the hybrid model behavior. To validate the capabilities of the proposed hybrid model, numerical simulation results are compared to a broad range of experimental data at different stress levels and strain rates; besides the consideration of two alloys in the present work, would demonstrate the model's capabilities and flexibility to model multiple alloys behavior. Finally a quantitative analysis is provided to determine the percentage error and coefficient of determination between the experimental data and numerical simulation results to estimate the efficiency of the proposed hybrid model. 


\section{TABLE OF CONTENTS}

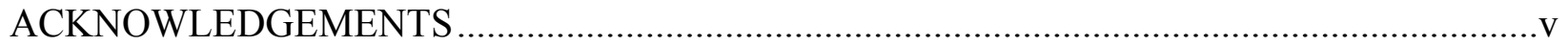

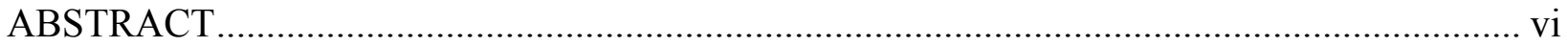

TABLE OF CONTENTS ............................................................................................. viii

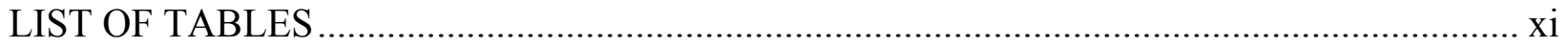

LIST OF FIGURES .................................................................................................

CHAPTER 1: INTRODUCTION ....................................................................................

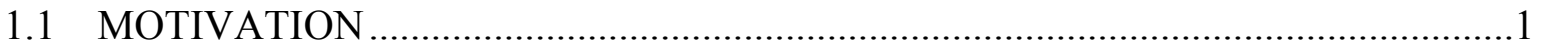

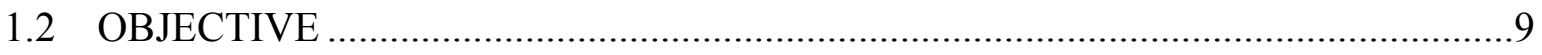

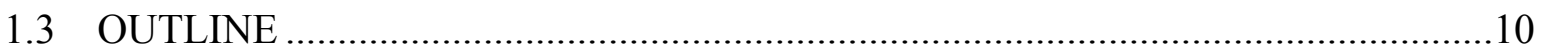

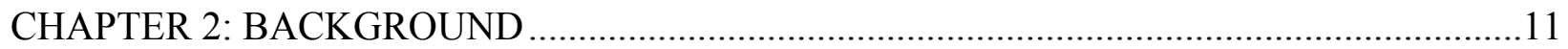

2.1 FUNDAMENTALS OF VISCOPLASTICITY ................................................... 11

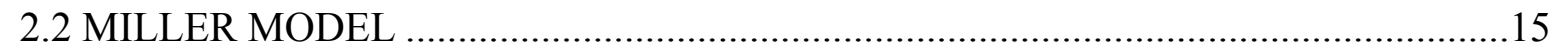

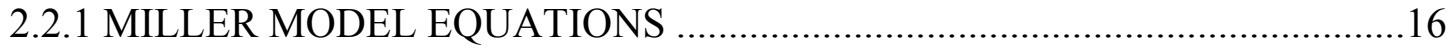

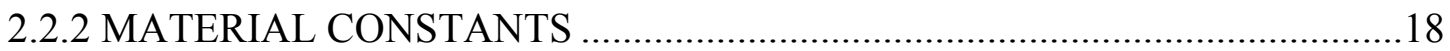

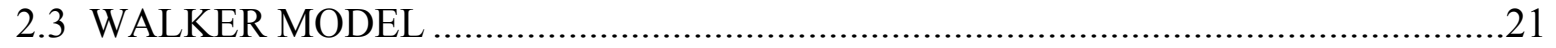

2.3.1 WALKER MODEL EQUATIONS ..........................................................22 


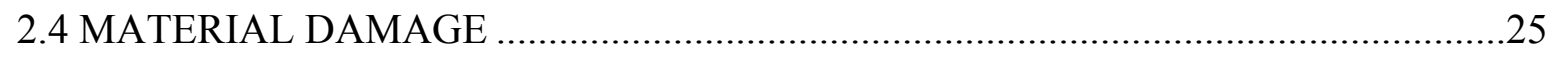

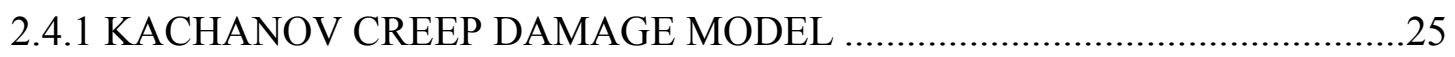

2.4.2 SIN-HYPERBOLIC CREEP DAMAGE MODEL ………………………........27

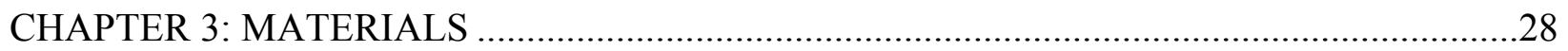

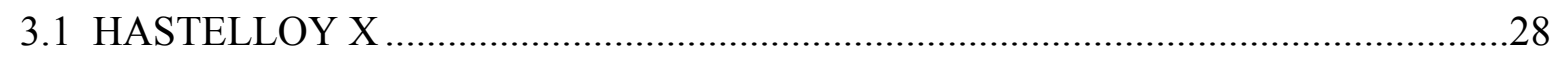

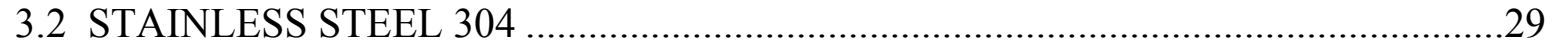

CHAPTER 4: NUMERICAL OPTIMIZATION SOFTWARE …………………….....................30

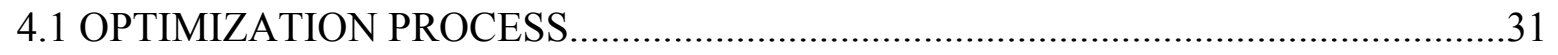

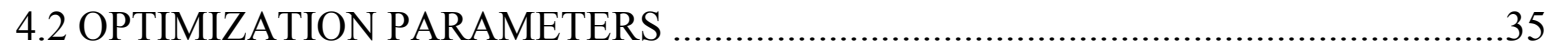

CHAPTER 5: EXERCISE OF MILLER AND WALKER MODELS ..........................................36

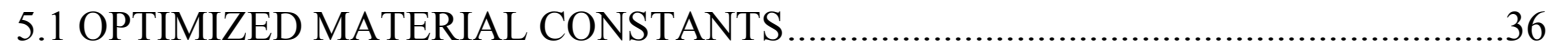

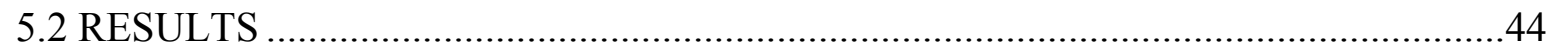

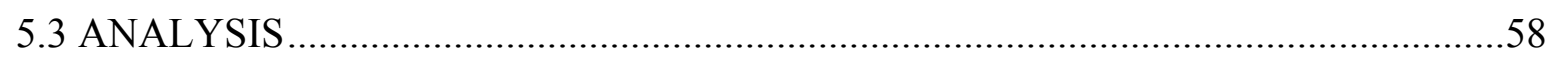

CHAPTER 6: DEVELOPMENT AND EXERCISE OF A NOVEL HYBRID UNIFIED

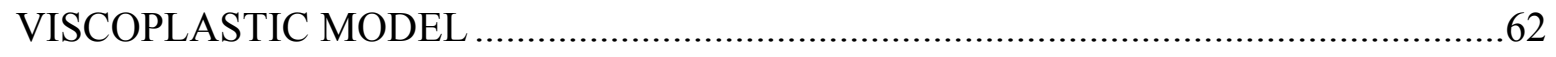

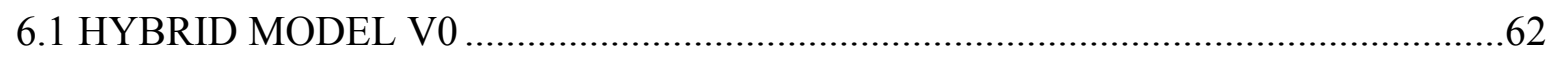

6.1.1 MODEL DEVELOPMENT .....................................................................63

6.1.2 EXERCISE OF HYBRID MODEL V0 ...........................................................64 


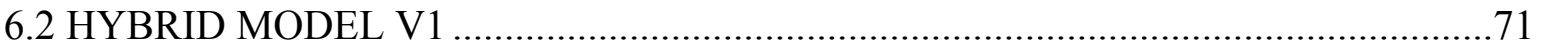

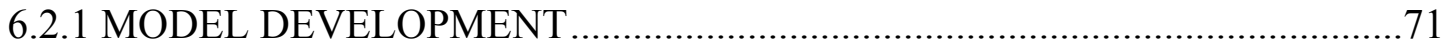

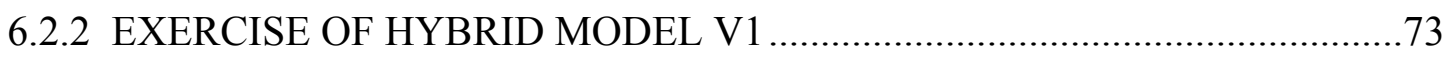

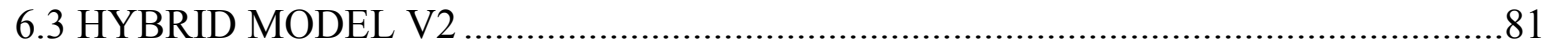

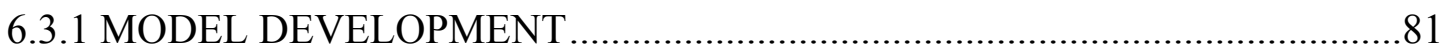

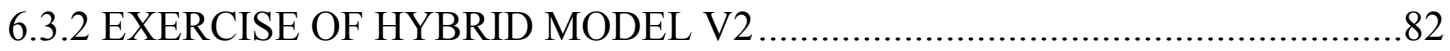

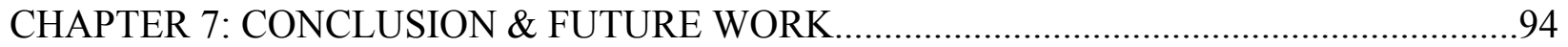

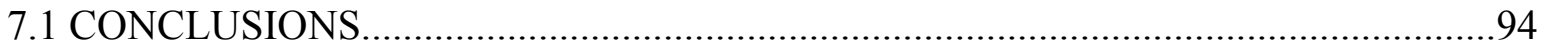

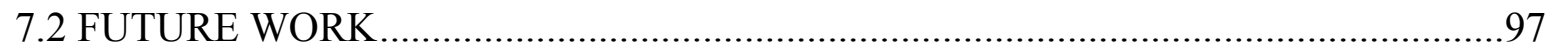

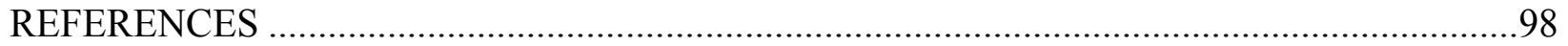

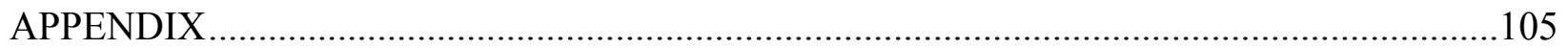

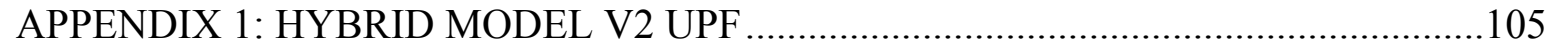

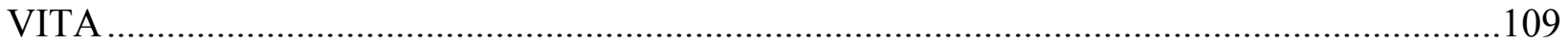




\section{LIST OF TABLES}

Table 1: Hastelloy X chemical composition (weight. \%) [32] .............................................. 28

Table 2: stainless steel 304 chemical composition (weight. \%) [68]..................................... 29

Table 3: Miller model initial guess and optimized material constants for Hastelloy X creep...... 38

Table 4: Walker model initial guess and optimized material constants for Hastelloy X creep .... 39

Table 5: Miller model initial guess and optimized material constants for stainless steel $304 \ldots . . .43$

Table 6: Walker model initial guess and optimized material constants for stainless steel $304 \ldots . .43$

Table 7: Calculated mean percentage error (MPE) and coefficient of determination $R 2$ for Miller

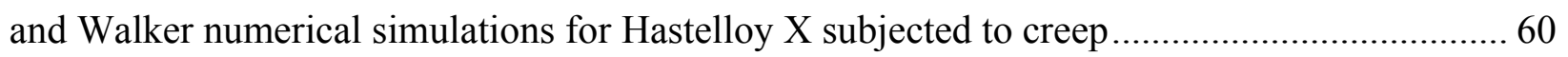

Table 8: Calculated mean percentage error (MPE) and coefficient of determination $R 2$ for Miller and Walker numerical simulations for stainless steel 304 subjected to low cycle fatigue .......... 61

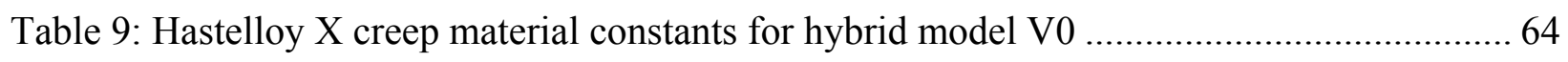

Table 10: 304 Stainless steel low cycle fatigue material constants for hybrid model V0 ........... 65

Table 11: Mean percentage error (MPE) and coefficient of determination $(R 2)$ of Hastelloy X

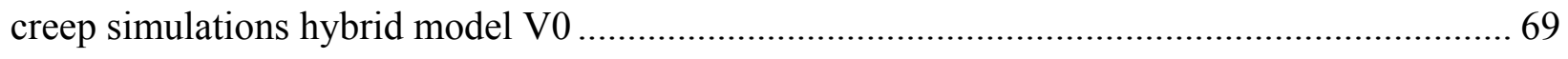

Table 12: Mean percentage error (MPE) and coefficient of determination $(R 2)$ of 304 stainless steel data low cycle fatigue simulations hybrid model V0 ............................................... 70

Table 13: Rest stress rate equations tested for improvement............................................... 72

Table 14: Hastelloy X Creep Material Constants for hybrid model V1 ................................. 73

Table 15: Stainless Steel 304 Fatigue Material Constants for hybrid model V1 ...................... 74

Table 16: Mean percentage error (MPE) and coefficient of determination $(R 2)$ of Hastelloy X

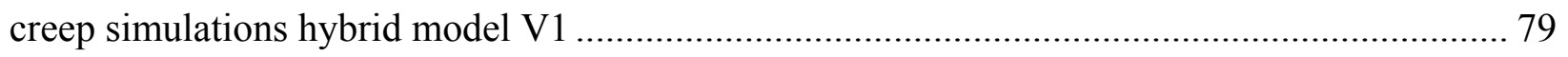


Table 17: Mean percentage error (MPE) and coefficient of determination (R2) of 304 stainless

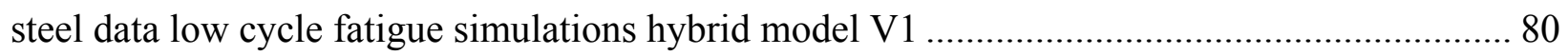

Table 18: Hastelloy X creep material constants for hybrid model V2 ………………................. 83

Table 19: 304 Stainless Steel Fatigue material constants for hybrid model V2 …….................... 84

Table 20: Mean percentage error (MPE) and coefficient of determination $(R 2)$ of Hastelloy X

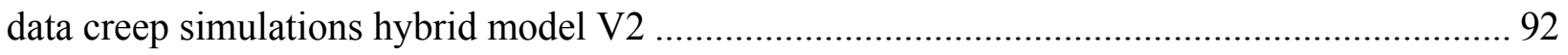
Table 21: Mean percentage error (MPE) and coefficient of determination $(R 2)$ of 304 stainless

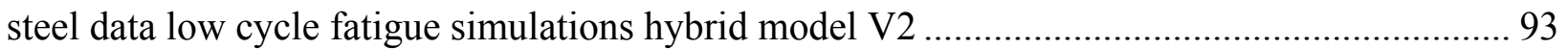




\section{LIST OF FIGURES}

Figure 1: NGNP configuration 1 (direct electrical cycle and a parallel IHX) ........................... 2

Figure 2: NGNP configuration 2 (direct electrical cycle, parallel IHX, and SHX) .................... 3

Figure 3: NGNP configuration 3 (indirect electrical cycle and a parallel SHX) ....................... 3

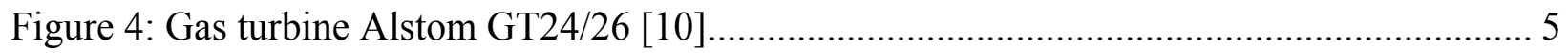

Figure 5: W501 F Gas turbine components (a) combustor, (b) transition pieces, (c) Alstom

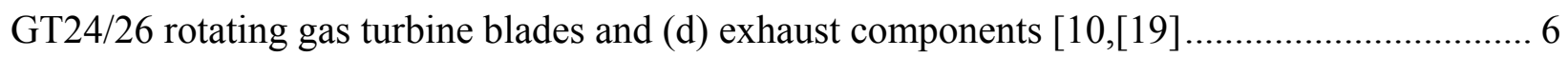

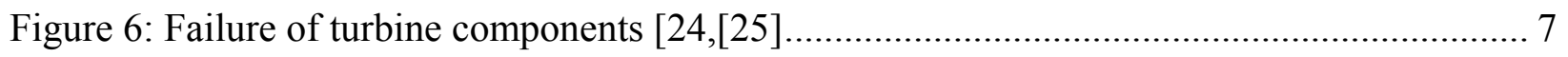

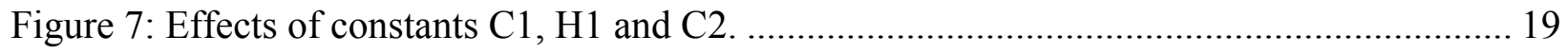

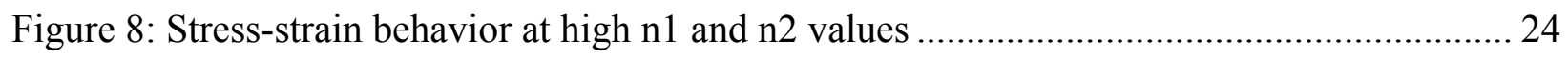

Figure 9: MACHO material constant optimization process ............................................. 34

Figure 10: Objective Function results of the optimization process of Miller and Walker models

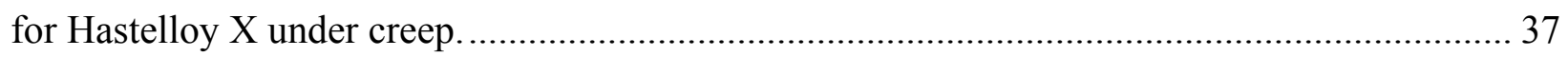

Figure 11: Objective function results of the optimization process of Miller and Walker models

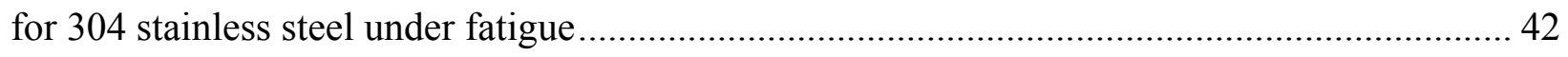

Figure 12: Miller model simulation vs. experimental data [32] .......................................... 48

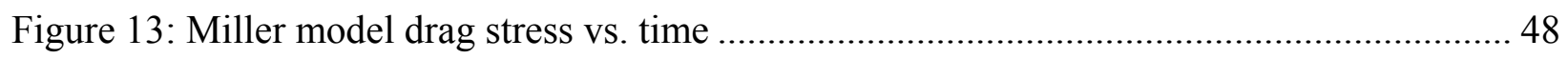

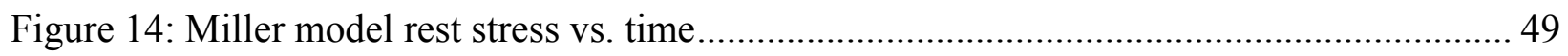

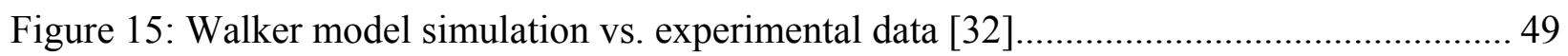

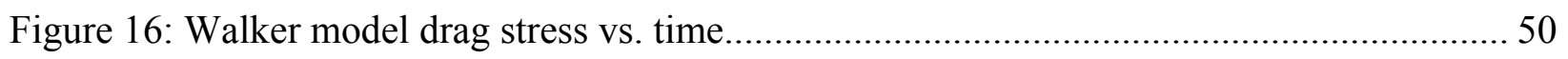

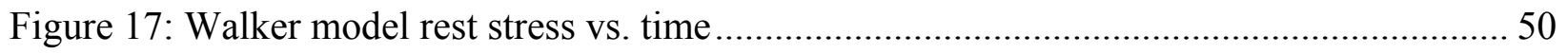

Figure 18: Miller model simulation vs. experimental data [68] .......................................... 52

Figure 19: Miller model simulation vs. experimental data [68] ........................................... 52 
Figure 20: Walker model simulation vs. experimental data [68].............................................. 54

Figure 21: Walker model simulation vs. experimental data [68]............................................. 54

Figure 22: Comparison of stress amplitude vs. number of cycles at $\Delta \varepsilon=0.005 \ldots \ldots \ldots \ldots \ldots \ldots \ldots \ldots \ldots . . .57$

Figure 23: Comparison of stress amplitude vs. number of cycles at $\Delta \varepsilon=0.007 \ldots \ldots \ldots \ldots \ldots \ldots \ldots \ldots \ldots \ldots . . .57$

Figure 24: Hastelloy X creep vs hybrid model V0 simulation at multiple stress levels ............... 67

Figure 25: 304 Stainless steel low cycle fatigue vs. hybrid model V0 simulation at $\Delta \varepsilon=0.005 \ldots 68$

Figure 26: 304 Stainless steel low cycle fatigue vs. hybrid model V0 simulation at $\Delta \varepsilon=0.007 \ldots 68$

Figure 27: Hastelloy X creep experimental data vs. Hybrid model V1 simulated data................. 75

Figure 28: Hastelloy X creep experimental data vs. Hybrid model V1 simulated data.................. 76

Figure 29: Stainless Steel 304 experimental data vs. Hybrid model V1 simulated data. .............. 77

Figure 30: Stainless Steel 304 experimental data vs. Hybrid model V1 simulated data. ............. 78

Figure 31: Hastelloy X creep experimental data vs. Hybrid model V2 simulated data................. 86

Figure 32: Hastelloy X creep experimental data vs. Hybrid model V2 simulated data................ 86

Figure 33: Stainless Steel 304 experimental data vs. Hybrid model V2 simulated data. .............. 88

Figure 34: Stainless Steel 304 experimental data vs. Hybrid model V2 simulated data. .............. 89

Figure 35: Comparison of stress amplitude and number of cycles at $\Delta \varepsilon=0.005$......................... 91

Figure 36: Comparison of stress amplitude and number of cycles at $\Delta \varepsilon=0.007 \ldots \ldots \ldots \ldots \ldots \ldots \ldots \ldots . . . .91$ 


\section{CHAPTER 1: INTRODUCTION}

\subsection{Motivation}

Nuclear and chemical reactors are now being used to generate electricity with clean methods without the emission of greenhouse gases [1]. Some examples of these types of reactors are: the Very-High Temperature Reactor (VHTR) which is the Next Generation Nuclear Plant (NGNP) in the United States [2], the High Temperature Test Reactor (HTTR) in Japan [3], Nuclear Hydrogen Development and Demonstration plant in Korea [4], and the High temperature Gas Reactor in Japan [5]. The efficiency of these reactors is related to the temperature at which they operate; therefore, high operating temperatures are desired for better efficiencies. During operation at high temperature, the reactor requires cooling systems to keep it within safe operating temperature ranges. The coolant system is formed by the intermediate heat exchanger (IHX), which exchange heat between the primary heated coolant that come directly from the reactor primary system to a secondary working fluid which cools down the primary coolant [13]. Three design configuration recommended by Davis et al. [6] for NGNP are presented in Figure 1-Figure 3 [7]. NGNP proposed configuration 1 is presented in Figure 1, this configuration requires the smallest IHX design and produces the highest overall electrical power production efficiency for the NGNP. In the design configuration 1, the designed operating conditions are: reactor outlet temperature is in the range of $850^{\circ} \mathrm{C}-950^{\circ} \mathrm{C}$, the IHX inlet pressure of 7.0 $\mathrm{MPa}$ and outlet pressure of $6.95 \mathrm{MPa}, \mathrm{IHX}$ inlet temperature is $850^{\circ} \mathrm{C}-950^{\circ} \mathrm{C}$ and outlet temperature of $523^{\circ} \mathrm{C}-628^{\circ} \mathrm{C}$. Proposed configuration 2 is presented in Figure 2; this configuration is similar to configuration 1, but includes a third loop which adds a separation between the NGNP and a hydrogen generation process. In the design configuration 2 the reactor 
outlet temperature is in the range of $900^{\circ} \mathrm{C}-950^{\circ} \mathrm{C}$, with IHX inlet pressure of $7.0 \mathrm{MPa}$ and outlet pressure of $6.95 \mathrm{MPa}$, IHX inlet temperature is $900^{\circ} \mathrm{C}-950^{\circ} \mathrm{C}$ and outlet temperature of $543^{\circ} \mathrm{C}-593^{\circ} \mathrm{C}$. Finally the design configuration 3 is presented in Figure 3; this configuration requires the larger IHX design since it must transfer all the reactor thermal power to the external power conversion system and hydrogen generation process. In design configuration 3 the reactor outlet temperature is in the range of $950^{\circ} \mathrm{C}-950^{\circ} \mathrm{C}$, IHX inlet pressure of $7.0 \mathrm{MPa}$ and outlet pressure of $6.95 \mathrm{MPa}, \mathrm{IHX}$ inlet temperature of $850^{\circ} \mathrm{C}-950^{\circ} \mathrm{C}$ and outlet temperature of $484^{\circ} \mathrm{C}$ $584^{\circ} \mathrm{C}[7]$.

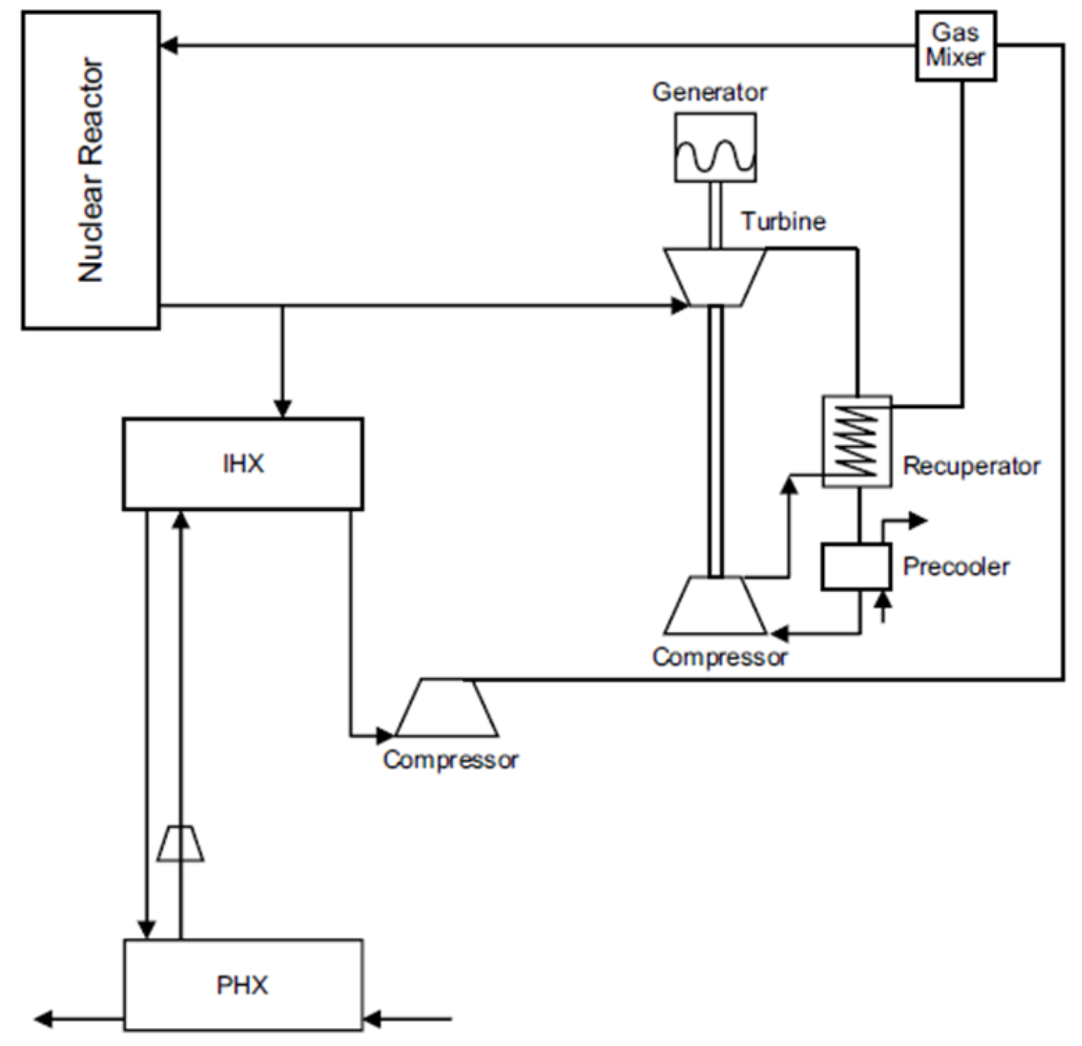

Figure 1: NGNP configuration 1 (direct electrical cycle and a parallel IHX) 


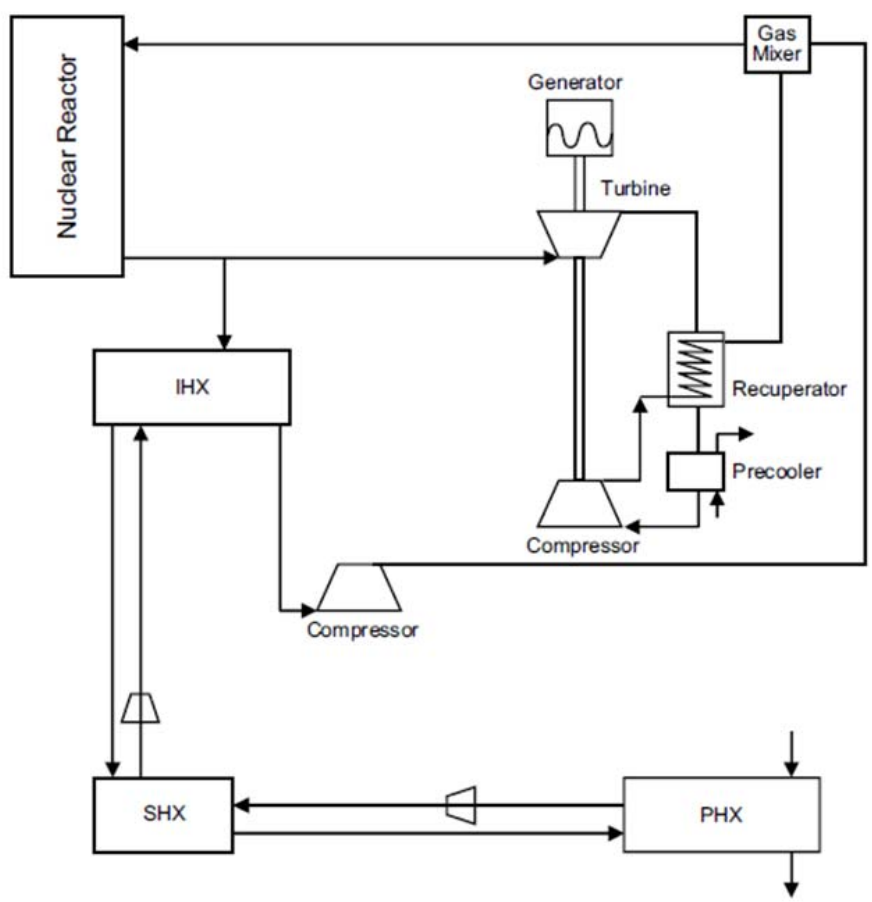

Figure 2: NGNP configuration 2 (direct electrical cycle, parallel IHX, and SHX)

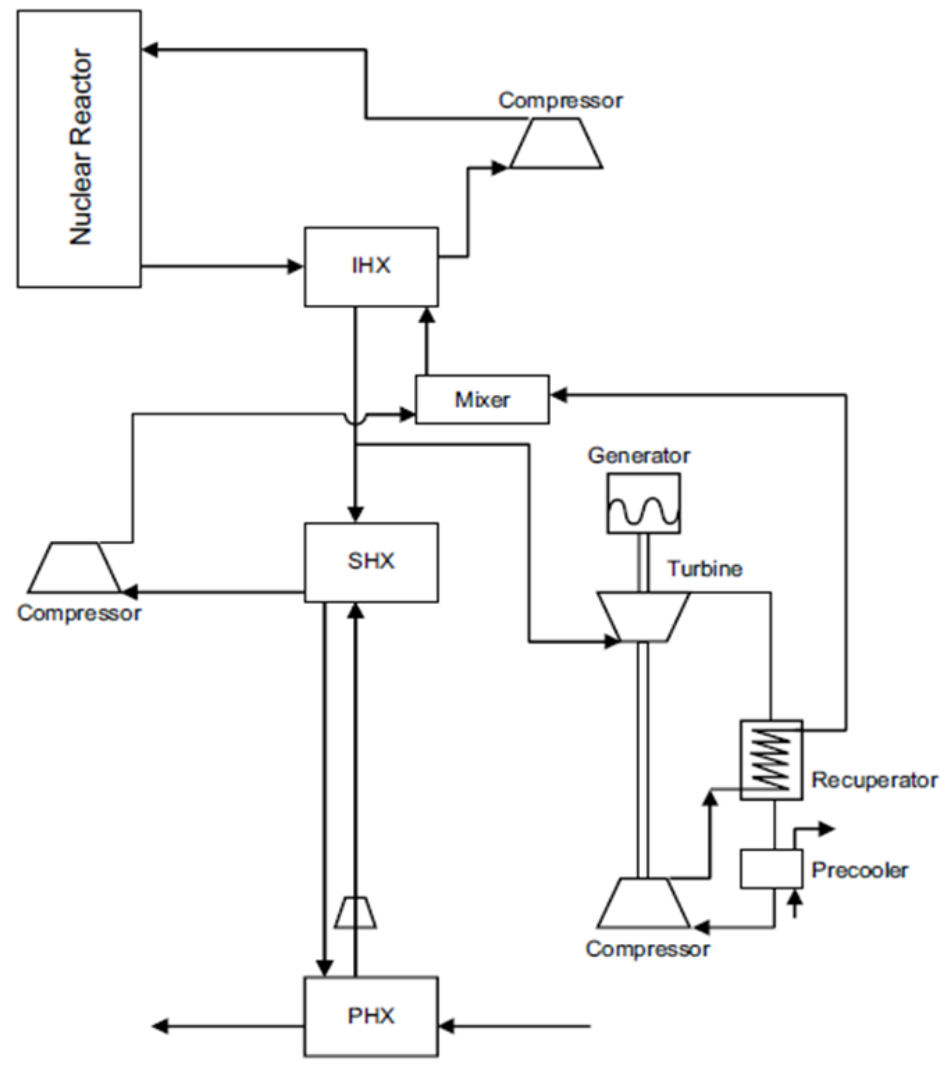

Figure 3: NGNP configuration 3 (indirect electrical cycle and a parallel SHX) 
From the previous described design configurations, it can be observed how in configuration 1 the IHX operates at higher temperatures; thus, resulting in the most efficient configuration for the NGNP plant, meaning that the higher the operating temperature the higher efficiencies the system results on. Hence, the IHX perform a crucial function and its optimal performance is vital to maintain a safe temperature and a high efficiency of the reactor; consequently, accurate IHX piping component design is pivot to achieve high quality designs of IHX piping. Therefore it is essential to have a deep understanding of the mechanical behavior of the constituent material used in the components under service-like conditions [8]. Since the IHX is operating at high temperature and extreme conditions, a material able to deal with thermal and mechanical stresses, corrosion, oxidation, and exhibit a high strength at elevated temperature is required for IHX piping components.

Gas turbines are extensively used for generation of electricity in power plants, and for propulsion in aerospace industry [9] (Figure 4). This kind of turbo machinery operates at elevated temperatures of above $1100^{\circ} \mathrm{C}$, and high pressure ratios of up to 23 to 1 for periods of more than 25,000 hours [11-13]. The Thermomechanical efficiency of gas turbines is dependent on the temperature at which the turbine operates. A high firing temperature leads to a dual benefit, an increase in specific work (that is the output per unit of air flow), and a reduction in fuel consumption [14-17]. These severe operation conditions produce multiple deformation mechanisms, which are applied to the turbine components such as the combustors, transition pieces, exhaust, and turbine blades $[13,18]$ illustrated in Figure 5. The purpose of the combustor is to mix the compressed air coming from the compressor with the fuel, and ignite the air-fuel mixture at temperatures of above $1500^{\circ} \mathrm{C}$. An example of a gas turbine combustor is provided in Figure 5 (a). 


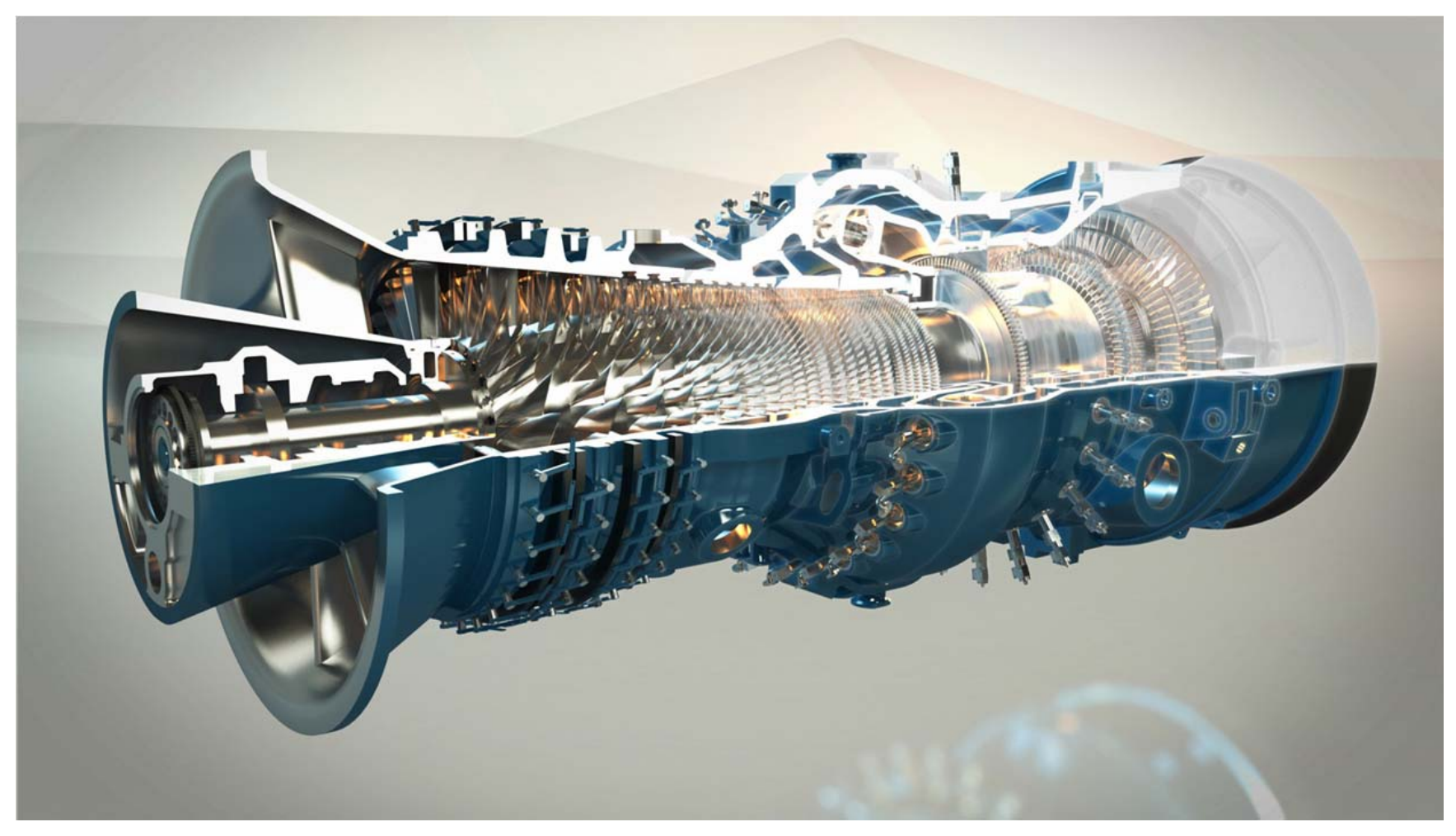

Figure 4: Gas turbine Alstom GT24/26 [10]

After the combustion process is done the combustion gases left the combustor and then enter the transition pieces which direct the gases against the nozzle guide vanes. An example of transition pieces is given in Figure 5 (b). The purpose of the nozzle guide vanes is to direct the combustion gasses to intersect the rotating turbine blades at the optimum angle. An example of rotating turbine blades is presented in Figure 5 (c). These components capture the combustion gases momentum causing the rotor to spin therefore generating power, Figure 5 (d) shows an example of exhaust components, which are responsible of directing the combustion gases out of the turbine once they passed through all the rotating turbine blades rows [20]. Some damage mechanisms are thermal stresses which are caused by the elevated operational temperature; mechanical stresses caused by the pressure differences and the rotational movement of components, creep which is caused by the long operation periods of the turbine, low and high 


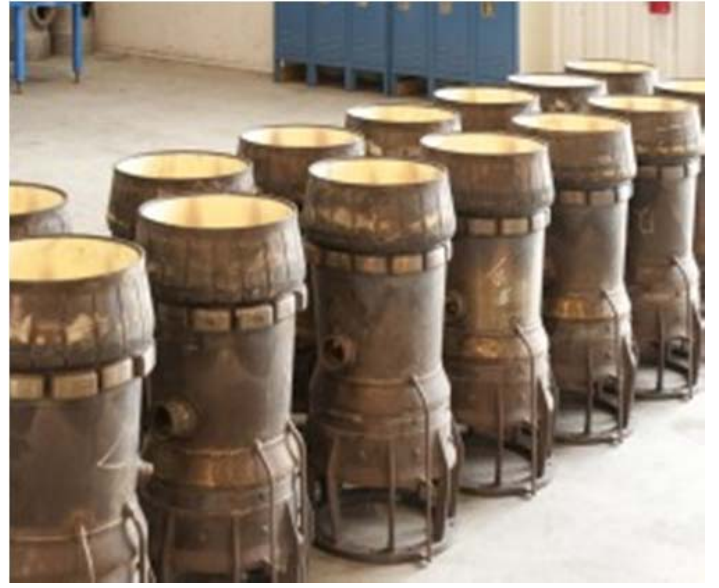

(a)

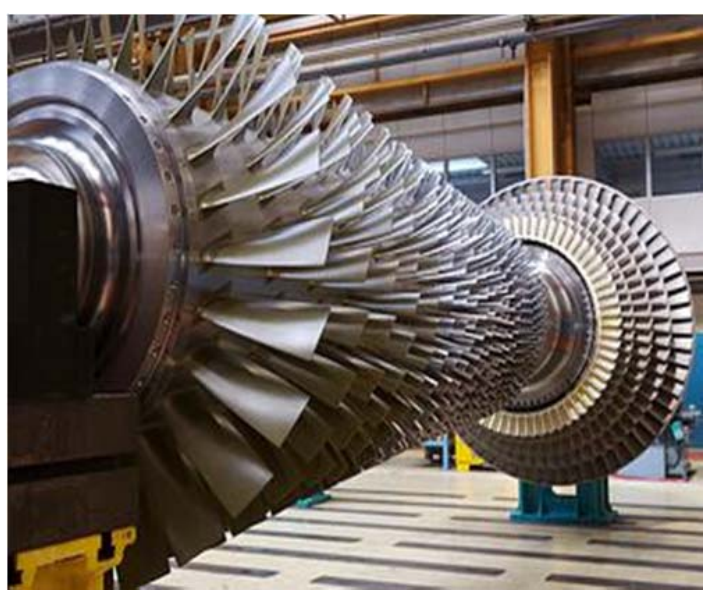

(c)

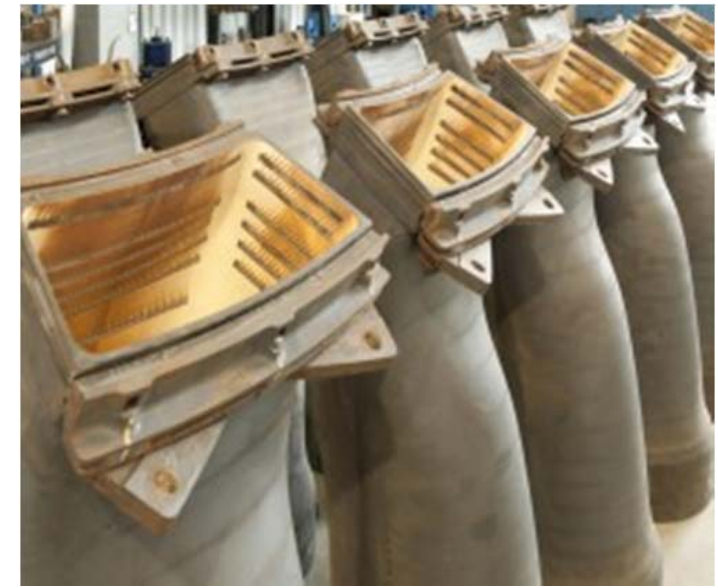

(b)

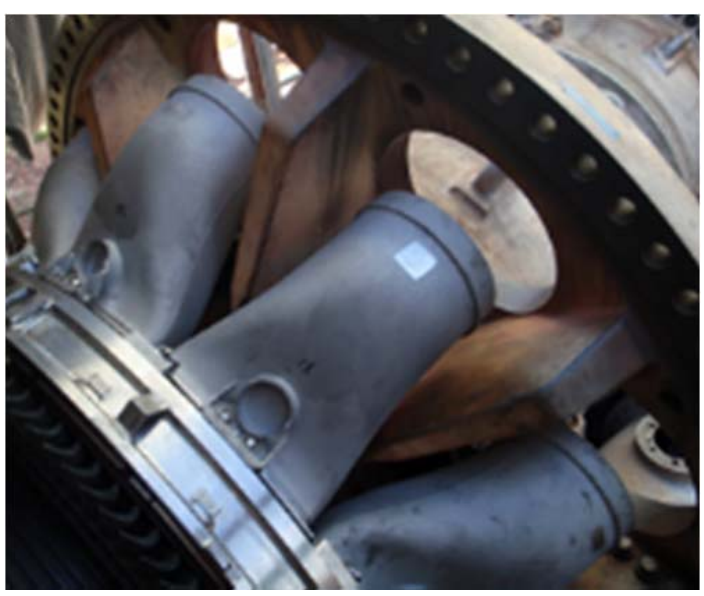

(d)

Figure 5: W501 F Gas turbine components (a) combustor, (b) transition pieces, (c) Alstom GT24/26 rotating gas turbine blades and (d) exhaust components [10,[19]

cycle fatigue caused by vibrations, the start-up, service time and shut-down of the equipment, oxidation caused by the contact of components to oxygen during operation. Depending on the damage stage caused by the mentioned mechanisms, they could lead to failure of the gas turbine components [21-23], which might be catastrophic as shown in Figure 6. The severe operation conditions of gas turbines create a situation where material selection for components' design 
plays a fundamental role to warranty components reliability. High strength materials are necessary to provide long components' life under elevated temperature [26-28].

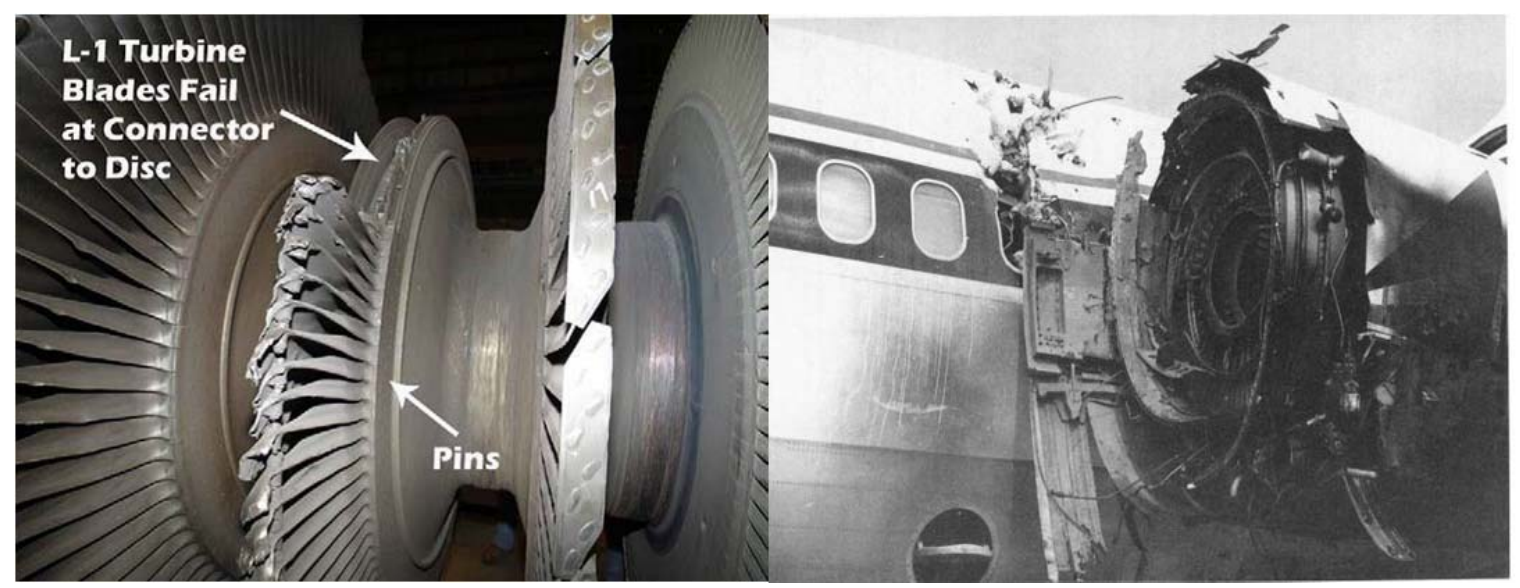

Figure 6: Failure of turbine components [24,[25]

For the previously described applications (IHX and gas turbine applications) good material candidates are austenitic, hardened superalloys [29]. The nickel-based superalloy Hastelloy X is an attractive candidate material. It is favored for these applications because of its high nickel content, which provides it with excellent mechanical properties at high temperature, high resistance to creep, oxidation and corrosion. A second candidate material for these type of applications is the austenitic stainless steel 304, which also possess high strength at elevated temperature [30-37]. However at elevated temperature these superalloys exhibit a viscoplastic (rate-dependent) behavior [38]. The nonlinear (viscoplastic) behavior of the material difficult the prediction of the material's response to loading.

In order to have an optimal design of gas turbine and IHX components, a detailed modeling of the material's behavior under any loading condition is essential to ensure the design integrity and quality of the component. Moreover, by knowing the behavior of the material the operational temperature might be increased for better system efficiencies. On the other hand, a better understanding of the material behavior leads to less conservative designs which in return reduce 
the cost of hardware and components, since the material is effectively used $[15-17,39]$. There has been considerable effort to develop unified constitutive models capable of describing the inelastic behavior of Hastelloy X and stainless steel 304. These "unified" models are designed to model the multiple deformation mechanisms present during various loading cases such as stress relaxation, monotonic tension, creep and fatigue. Historically, numerous viscoplastic models have been proposed in literature such as Chaboche, Bodner, Hart, Miller, Walker, BodnerPartom among many others [40-44]. Viscoplastic constitutive models are shaped by material constants, which are characteristic of each material. Material constants are typically calculated using specific types of experimental data. The complexity of the model equations and the considered temperature ranges dictates the total number of material constants required for each model. The procedure to calculate these material constants is not well documented leaving gaps in the calculation process that leads to the "unsystematic" calculation of material constants. This unsystematic calculation of constants might result in improper usage of viscoplastic models. 


\subsection{OBJECTIVE}

The objectives of this thesis are as follows:

- Test the recently developed Material Constant Heuristic Optimizer (MACHO) software to calculate material constants and perform finite element $(\mathrm{FE})$ numerical simulations.

- Use two different alloys (Hastelloy X and stainless steel 304) to validate the ability of $\mathrm{MACHO}$, the considered and proposed models to describe the behavior of multiple materials.

- Analyze and Exercise the Miller and Walker unified viscoplastic models, to determine the most accurate model describing the inelastic deformation of Hastelloy X under creep and the inelastic deformation of 304 stainless steel under low cycle fatigue. Conduct a series of creep and low cycle fatigue numerical simulations at a broad range of stress levels and strain amplitudes to fully evaluate the capabilities and limitations of each model. Compare the numerical simulation results to experimental data, and estimate the goodness of fit between them. Formulate a qualitative conclusion on which model better predicts the material's behavior.

- Develop a hybrid unified viscoplastic constitutive model based on the best aspects of Miller and Walker unified viscoplastic models. Add a damage term to the hybrid model equations to account for damage state and determine the reaming life of the material. Explain the meaning and impact of each material constant on the constitutive equations. Perform a series of creep (for Hastelloy X) and fatigue (for stainless steel 304) numerical simulations at a broad range of stress levels and strain amplitudes to fully evaluate the capabilities and limitations of the proposed model. Compare the numerical simulation results to an exhaustive database of experimental data, and determine the goodness of fit between them by the percentage error and coefficient of determination. 


\subsection{OUTLINE}

The work is organized as follows. In chapter 2 a brief review on unified viscoplastic models is provided, where the basic components and basic skeleton of a viscoplastic model are explained. Besides, the Miller and Walker viscoplastic models are presented along with a explanation on material damage. Chapter 3 introduces the considered superalloys Hastelloy X and stainless steel 304; besides, their chemical composition and their mechanical properties are discussed. In Chapter 4 the use of the MAterial Constant Heuristic Optimizer (MACHO) software to systematically determine material constants for each constitutive model is explained, along with a discussion on the optimization process. In chapter 5 a comparative analysis between Miller and Walker constitutive model ability to predict superalloys behavior is presented. In this chapter both constitutive models are exercised to model the creep behavior of Hastelloy X at broad range of stress levels and the behavior of stainless steel 304 under low cycle fatigue at different strain amplitudes. In chapter 6 the development of a hybrid constitutive model is presented. A detail explanation of the equations is provided, along with a discussion of the material constants and its impact in the constitutive equations. the hybrid model development is presented in three sucessive stages. Finally, in chapter 7 conclusions are formulated based on the model's performance and future work is suggested. 


\section{CHAPTER 2: BACKGROUND}

\subsection{FUNDAMENTALS OF VISCOPLASTICITY}

A unified viscoplastic models is a mathematical model capable of describing and/or predict a material's behavior under complex loading conditions at elevated temperatures. It is called a unified model because contrary to classical theories of inelastic deformation, it is unifying the creep and plastic strain into one unique inelastic strain term; the unification of this term allows the modeling of multiple loading conditions (creep, monotonic tension or cyclic loading) by using the same set of constitutive equations for any loading condition. These types of models are based in the theory of time-dependent deformation $[45,46]$. However, the total mechanical strain rate $\dot{\varepsilon}_{m e c h}$ of the material is represented by the summation of the elastic strain rate $\dot{\varepsilon}^{e}$ and the inelastic strain rate $\dot{\varepsilon}^{i}$ Eq. (1); where the elastic strain rate is calculated using Hook's law and the inelastic strain rate is calculated using the unified viscoplastic model.

$$
\dot{\varepsilon}_{\text {mech }}=\dot{\varepsilon}^{e}+\dot{\varepsilon}^{i}
$$

Although proposed unified viscoplastic models differ in number of conforming material constants, mathematical function used or effectiveness most models share several format details and basic structure. According to Chaboche [47] the basic structure of unified viscoplastic models should contain a flow law and hardening law equations to describe the evolution of the inelastic strain rate and the state variables. A basic skeleton of the constitutive equations of this type of models is presented in Eq. (2) - Eq. (4) [48]. Usually these equations are stiff non-linear differential equations, meaning that a minimum change in one constant or term can considerably affect the solution of equations; thus, these equations are usually difficult to solve and complicated integration methods are used to resolve them. The flow law, Eq. (2) describes the 
inelastic strain rate as a function of the applied stress $\sigma$ and the state variables that describe the hardening state of the material. Usually the strain rate equation contains a power law function or a hyperbolic sine function within its structure; besides the signum (sgn) function is added to account for reverse stress flow in case of opposite direction loading. The hardening law refers to the use of state variables to determine the strain state within the material due to the applied stress and the environment conditions, such as temperature. This hardening law is divided into kinematic and isotropic hardening [49]. Kinematic hardening $R$ refers to the back or rest stress, which describes the global effects of repulsive forces among dislocations in pileups against obstacles; if a yield criterion is consider kinematic hardening accounts for the translation of the yield surface in the stress space, this accommodates the Bauschinger effect. Equation (3) describes the kinematic hardening with respect to time. Isotropic hardening $D$ refers to the drag stress, which describes the drag forces that resist dislocation motion; if yield criterion is consider, then isotropic hardening accounts for the change in size of the yield surface in the stress space. Isotropic hardening with respect to time is represented by Eq. (4) [48].

$$
\begin{gathered}
\dot{\varepsilon}^{i}=K\left(\frac{\sigma-R}{D}\right) \\
\dot{R}=X_{1} \dot{\varepsilon}^{i}-X_{2} R\left|\dot{\varepsilon}^{i}\right|-X_{3}|R| \\
\dot{D}=Z_{1} \dot{\varepsilon}^{i}-Z_{2} D\left|\dot{\varepsilon}^{i}\right|-Z_{3}|D|
\end{gathered}
$$


Usually hardening equations follow the hardening-recovery format. Terms $X_{1}$ and $Z_{1}$ represent the hardening terms of the model, these terms are function of the inelastic strain rate, meaning that they are activated only in the presence of inelastic strain rate. The presence of hardening is mainly due to the block of dislocation motion. Terms $X_{2}$ and $Z_{2}$ represent the dynamic recovery capability of the model. The dynamic recovery is active due to the presence of an inelastic strain rate which allows mainly the climbing and cross-slip of dislocations which allows dislocation motion therefore recovering some hardening. Terms $X_{3}$ and $Z_{3}$ represent the effects of static thermal recovery; these terms are not function of the inelastic strain rate, they are activated by temperature. Once certain activation temperature is reached then the recovery term is activated and some hardening is recovered, the activation temperature is depended of the material and the recovery phenomena [48].

The $K, X$ and $Z$ terms in the constitutive equations represent material constants; these material constants are characteristic of each material, and depending on the model they might be characteristic of each temperature level. The total number of material constants vary with each model, normally the accuracy of the model is related to the number of material constants; a model with numerous constants is expected to have a high accuracy. The material constants values are determined from creep and fatigue experimental data. Each model has a different method to calculate material constants, for example some models require the use of external equations to calculate, other require the plotting of experimental data in a certain way or some other is just trial and error. The material constants finding process is not always an easy process, especially if the model consists of many material constants since this increase the calculation time. 
Even though unified viscoplastic models have been a promising solution to predict metallic material response during many years and numerous models have been proposed in literature, according to Chang and Thompson [50] there are still some shortcomings present in unified viscoplastic models. Some of them are (a) the proposed models have not been fully tested under rigorous conditions, therefore its full functionality have not been ensured (b) The material constants calculation methods are unclear since they are not well documented in all cases (c) As mentioned before the constitutive equations present a stiff regime, therefore its solution is not easy for time-dependent analysis. In order to take full advantage of these models and its capabilities, the aforementioned shortcomings must be overcome. So Even though unified viscoplastic models are not a new concept, there are still some empty spaces that need to be filled. 


\subsection{MILLER MODEL}

Miller model is a unified viscoplastic model also called MATMOD, proposed in 1975 by Alan K. Miller to model the viscoplastic behavior of materials subjected to high temperatures and high loads [51]. Miller developed this model considering four important factors. Factors which would classify it as a feasible or unfeasible model; the influence factors were: breadth, accuracy, realism and manageability. Breadth came in from the necessity of having a model with the ability to describe material's behavior at different temperatures and strain ranges, besides being applicable to as many materials as possible. Accuracy and realism were obvious goals since this is a scientific model. Finally manageability was a concern because the equations would have to be accessed many times during a finite element analysis; so the use of simple equations would help to keep low computation times. This model was developed trying to compromise among these four factors to reach an optimum model.

This model was developed based on the physical mechanisms that take place within the material during exposure to inelastic conditions. The considered physical mechanisms include dislocation pileups, dislocation tangles and their bowing effects, solutes atoms and dislocations interaction, sub-grain strengthening, and their thermal activation. According to its creator, this approach will lead to the best solution because the deformation model relies on the same microstructural events that cause the deformation [52]. Individual mathematical consideration of each mechanism effects will be more accurate in an individual scale, but this model attempts to bring them together towards a more realistic structural analysis by the interaction among the inelastic mechanisms [53].

This unified model does not just consider creep and fatigue caused by the material's exposure to extreme conditions, its developer claims that it also has the capabilities of simulating multiple 
inelastic mechanisms such as: transient (primary) creep, steady-state (secondary) creep, monotonic short-time plastic deformation, cyclic hardening and softening, Bauschinger effect, rate effects, temperature effects, annealing, the accumulation and the history interaction of these effects with respect to time [53].

Miller model consists on a set of three rate dependent equations and a pair of auxiliary equations which introduce the temperature dependence modeling capability. Rest stress and drag stress equations represent the rates of change of these two state variables with respect to time. The model's equations are conformed by eight main material constants which are characteristic of each material. The process to determine the constant's values involves the use of auxiliary equations and/or plotting experimental data, and changing the constant's values until the data reaches a desired region or desired shape [53]; this process is explained in section 2.2.2 of this chapter. The required experimental data comes from short-time monotonic, creep and cyclic loading tests at high temperatures.

\subsubsection{Miller Model EquATions}

The inelastic strain rate equation, Eq. (5) is based on Garofalo's steady state creep equation [51]; it was modified to include drag and rest stress variables. This equation is dependent on the applied stress $\sigma$, and the two state constants $R$ (rest stress) and $D$ (drag stress) [52]. The strain rate equation, Eq. (5) requires three material constants $B, \theta^{\prime}, n$; constant $\theta^{\prime}$ brings the temperature dependence into the model, $n$ represents the rate sensitivity of the stress and $B$ is a material constant.

$$
\dot{\varepsilon}=B \theta^{\prime}\left\{\sinh \left(\frac{|\sigma-R|}{D}\right)^{1.5}\right\}^{n} \operatorname{sgn}(\sigma-R)
$$


The second rate equation Eq. (6) describes the rate of kinematic hardening with respect to time. The first term in the rest stress rate equation represents the amount of hardening of the material, it is produced by the piling up of dislocations against obstacles; the second term represents the recovery that is produced by the climb and cross-slip of dislocations and by thermal recovery. The constants $H_{l}, B, A_{l}$ represent material constants; $\theta^{\prime}$ is the temperature dependence term which accounts for thermal recovery. The constant $H_{l}$ determines how rapidly the rest stress reaches a saturated value during cyclic loading. The purpose of the signum function sgn and the absolute values is to give the equation the ability to take into consideration reverse stress flow [52].

$$
\dot{R}=H_{1} \dot{\varepsilon}-H_{1} B \theta^{\prime}\left[\sinh \left(A_{1}|R|\right)\right]^{n} \operatorname{sgn}(R)
$$

The third differential Eq. (7) describes the rate of isotropic hardening with respect to time. In Eq. (7) the first term represents the hardening of the material and the second term represents the recovery of the material. During steady state the hardening and recovery terms are equal, therefore resulting in $\dot{D}=0$, the same is applicable to the rest stress equation, meaning that at steady state the inelastic strain rate is dependent on the steady state stress only. It is important to notice that the drag stress rate equation is dependent on rest stress $R$ and drag stress $D$; this dependence was establish to give this equation the ability to consider cyclic loading by limiting the amount of isotropic hardening due to drag stress. The constants $H_{2}, C_{2}, A_{2}$ are material constants.

$$
\dot{D}=H_{2}|\dot{\varepsilon}|\left[C_{2}+|R|-\left(A_{2} / A_{1}\right) D^{3}\right]-H_{2} C_{2} B \theta^{\prime}\left[\sinh \left(\mathrm{A}_{2} D^{3}\right)\right]^{n}
$$

As mentioned before the term $\theta^{\prime}$ represents the temperature dependence of the model and its value is given by Eq. (8) and/or Eq. (9) depending on the initial temperature. This dependence is 
due to this model's assumption that the apparent activation energy $Q$ is temperature dependent, constant at a temperature above $0.6 T_{m}$ (where $T_{m}$ represents the melting temperature) and variant at temperatures below. Variable $Q$ represents the activation energy of processes related to inelastic deformation that are thermally activated, $k$ represents the gas constant, and $T$ is the working temperature.

$$
\theta^{\prime}=\left\{\begin{array}{c}
\exp \left(\frac{-Q}{k T}\right) \text { For } T \geq 0.6 \mathrm{Tm} \\
\exp \left\{\frac{-Q}{0.6 k T_{m}}\left[\ln \left(\frac{0.6 T_{m}}{T}\right)+1\right]\right\} \text { For } T<0.6 T_{m}
\end{array}\right.
$$

\subsubsection{MATERial Constants}

As mentioned before model's equations are conformed by eight main material constants, experimental data coming from monotonic tension creep and cyclic loading testing is used in the calculation of material's constants values. It is important to solve for the constants in a specific order so that all the constants can be solved, this because the calculation process involves the use of previously solved constants. The solving process is the following.

The first step is to find the constants responsible for the steady state creep. The constant $Q$ and $\theta^{\prime}$ represents activation energy for plastic flow of the material at high temperature and temperature-dependent factor, respectively. These constants are calculated using creep test data

and plotting the minimum creep strain rate $\dot{\varepsilon}_{\text {ss }}$ against applied stress $\sigma_{s s}$ with temperature as a parameter. Then divide each strain rate by $\theta^{\prime}$ and try different values of $Q$ in the temperature factor Eq. (8) and Eq. (9) until all the minimum creep data for the different temperatures coalesce in a narrow rectangular area. These will be the best values of $Q$ and $\theta^{\prime}$. 
The second step is to find a value for the constant $A$, which is not clearly present in the model's equations but it is required to calculate constants $A_{1}$ and $A_{2}$. Constant $A$ is calculated by using Eq. (10) and using trial and error, until Eq. (9) results in a straight line when plotted $\log \left(\dot{\varepsilon}_{s s} / \theta^{\prime}\right)$ against $\log \left[\sinh \left(A \sigma_{s s}\right)\right]$. The slope of this straight line is what defines the value of $n$ and the value of $\dot{\varepsilon}_{s s} / \theta^{\prime}$ at $\log \left[\sinh \left(A \sigma_{s s}\right)\right]=1$ is the value of $B[55]$.

$$
\log \left(\dot{\varepsilon}_{s s} / \theta^{\prime}\right)=\log (B)+n \log \left[\sinh \left(A \sigma_{s s}\right)\right]
$$

The third step is to calculate the constants responsible for the cyclic stress-strain behavior which are $C_{1}, H_{1}$ and $C_{2}$. These constants are calculated from cyclic stress amplitude versus cyclic strain amplitude and by best-fit procedure as shown in Figure 7 [52]; the arrows indicate the effect of an increase in each constant.

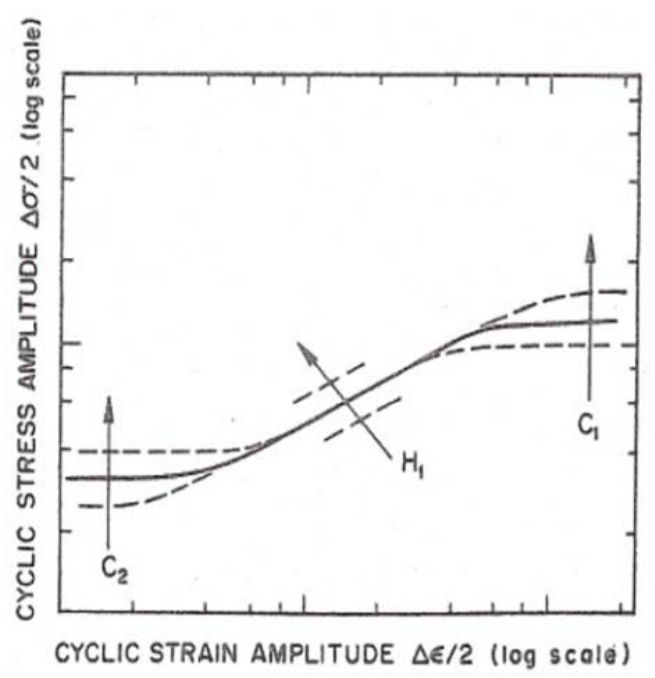

Figure 7: Effects of constants $C_{1}, H_{1}$ and $C_{2}$.

Constant $C_{l}$ controls the peak values of rest stress or stress amplitude by Eq. (11), where the rest stress is represented by $R_{s s}$ because that will be the point where stress reaches its steady state value due to cyclic saturation.

$$
R_{s s}=\left(A / A_{1}\right) \sigma_{s s}=C_{1} \sigma_{s s}
$$


Constant $H_{l}$ controls how fast the rest stress builds up, a high value of the constant will result in a fast building up of the rest stress causing high stress amplitude. So, different values of $H_{l}$ must be tried in Eq. (6) until the simulation data properly fits the experimental data. Constant $C_{2}$ sets the lower value for $D$. With a proper value for $C_{1}$, the values of $A_{1}$ and $A_{2}$ can be calculated using Eq. (12) and Eq. (13), which maintain a balance relation to $C_{l}$ and $A$. By using Eq. (14) where $Y$ is the $0.2 \%$ yield strength, the $D_{o}$ constant can be found. Constant $D_{o}$ represents the initial value for the history of the drag stress.

$$
\begin{gathered}
A_{2}=\left[A /\left(1-C_{1}\right)\right]^{3} \\
A_{1}=A / C_{1} \\
D_{o}=\left(\mathrm{Y}-.002 \mathrm{H}_{1}\right) /\left\{\sinh ^{-1}\left[\left(\frac{\dot{\varepsilon}}{B \theta^{\prime}}\right)^{\frac{1}{n}}\right]\right\} .0677
\end{gathered}
$$

Finally constant $H_{2}$ can be calculated by using tensile, creep or fatigue experimental data and tried different values of the constant in Eq. (6), so that simulation data fits experimental data. From the model equations it can be seen that Miller model is stress, temperature, structure and time dependent. Simple but many experimental data is required to solve for its constants. This model is capable of describing multiple inelastic deformations conditions by using these three differential equations. In the present work the temperature dependence function has been deactivated by setting the activation energy $Q=0$ which results in a temperature factor value of $\theta^{\prime}=1$. The deactivation was done because it is not required on the present analysis, since only isothermal test are considered; moreover this assumption eliminates one unknown material constant, therefore simplifying the constant finding process for this model. 


\subsection{WALKER MODEL}

Walker model is a unified viscoplastic model, developed by Kevin P. Walker in 1981; originally this model was called functional theory. This model was published in both integral and differential from, in the present work only the differential form is considered. This model is intended to describe the inelastic behavior of metals subjected to extreme environments. Walker model has the capability of modeling Bauschinger effect, cyclic hardening and softening, creep, stress relaxation, strain rate and temperature effects [56]. Walker model describe the inelastic behavior of materials by using five equations, three rate equations and two linear equations. The first rate equation, the inelastic strain rate equation is responsible for the modeling of all kinds of inelastic strains; the second rate equation describes the rest stress rate (called equilibrium stress by Walker), which is a state variable, while the third describes the recovery behavior of the model. The first linear equation describes the second state variable of the model, the drag stress behavior, and finally, the second linear equation represents equality between inelastic strain rate and accumulated inelastic strain rate. In this model, the saturation of the rest stress is independent of strain rate at high strains. This is because the state variable equation contains both dynamic and static recovery terms.

The model equations are conformed by fourteen material constants which are calculated using cyclic hardening and softening experimental data. The process to solve for the material constants has not been found in literature; thus, numerical optimization software has been used in the present work, to determine the value of these constants. This optimization software is further explained in chapter 4. A fundamental characteristic of this model is the temperature dependence. There are no explicit temperature dependent terms involved in the equations. The temperature dependence of the model is incorporated within the material constants values, which 
are functions of temperature and must be experimentally determined for each temperature. Therefore the number of material constants increases dramatically with the number of considered temperature levels.

\subsubsection{WALKER MODEL EQUATIONS}

The strain rate equation, Eq. (15) is based on a power law and it represents the strain rate behavior with respect to applied stress $\sigma$, rest stress $R$ and drag stress $D$. Constant $B$ and $n$ are material constants, the sgn function and absolute value are present in this equation to give the ability to account for reversed stress flow.

$$
\dot{\varepsilon}^{n}=B\left(\frac{|\sigma-R|}{D}\right)^{n} \operatorname{sgn}(\sigma-R)
$$

The rest stress rate equation, Eq. (16) introduces kinematic hardening into the model and provides the ability to account for Bauschinger effect. The equation is integrated by the material constants $n_{1}, n_{2}, n_{3}$, temperature $T$, temperature rate $\dot{T}$, recovery relation $\dot{G}$, inelastic strain $\varepsilon^{n}$ and inelastic strain rate $\dot{\varepsilon}^{n}$. The growth law for the rest stress accounts for strain hardening with its first two terms, and for recovery effects with the last two terms which are dependent of the recovery relation.

$$
\dot{R}=\left(n_{1}+n_{2}\right) \dot{\varepsilon}^{n}+\frac{\partial n_{1}}{\partial T} \dot{T} \varepsilon^{n}-\left(R-R_{0}-n_{1} \varepsilon^{n}\right)\left(\dot{G}-\frac{1}{n_{2}} \frac{\partial n_{2}}{\partial T} \dot{T}\right)
$$

The recovery relation is given by Eq. (17) which represents a relationship between dynamic and static recovery of the material. The dynamic recovery term (first term of the equation) governs hardening recovery in the presence of inelastic strain rate. On the other hand, the static recovery term (second term of the equation) governs hardening recovery in the absence of an 
inelastic strain rate. The equation incorporates material constants $n_{3}, n_{4}, n_{5}, n_{6}, R, R_{s}$, $m$, strain magnitude $\varepsilon^{k}$ and strain rate magnitude $\dot{\varepsilon}^{k}$.

$$
\dot{G}=\left[n_{3}+n_{4} \exp \left(-n_{5} \varepsilon^{k}\right)\right] \dot{\varepsilon}^{k}+n_{6}{\frac{|R|^{m-1}}{\left|R_{S}\right|}}^{m}
$$

The original model proposed by Walker [56], contains temperature rate terms $\dot{T}$ in the rest stress rate equation. These terms are responsible for modeling rest stress changes due to temperature changes during non-isothermal tests. However, in the present work only isothermal tests are considered; therefore the temperature rate term $\dot{T}$ has been omitted from the model's equations, resulting in Eq. (18).

$$
\dot{R}=\left(n_{1}+n_{2}\right) \dot{\varepsilon}^{n}-\left(R-R_{0}-n_{1} \varepsilon^{n}\right) \dot{\mathrm{G}}
$$

The material constants $n_{1}$ and $n_{2}$ determine how fast the rest stress grows, until it reaches saturation. With large constants values, the rest stress will saturate so promptly that in theory it will saturate within the elastic region; in a hysteresis loop, this will cause a square behavior of the plot as shown in Figure 8. With intermediate values it will take longer for the rest stress to saturate and in a hysteresis loop this will cause a rounded behavior of the plot. With small values, the rest stress will slowly saturate and even rounded behavior will be exhibit in a hysteresis loop [56]. The drag stress equation, Eq. (19) introduces isotropic hardening into the model and provides the ability to account for cyclic hardening and softening of the material. Material variables $n_{7}$ and $k_{2}$ control the isotropic behavior of the model depending on the accumulated inelastic strain $\varepsilon^{k}$; therefore the precision of this variables is of extreme importance to accurately model isotropic hardening or softening of the material [57].

$$
D=k_{1}-k_{2} \exp \left(-n_{7} \varepsilon^{k}\right)
$$




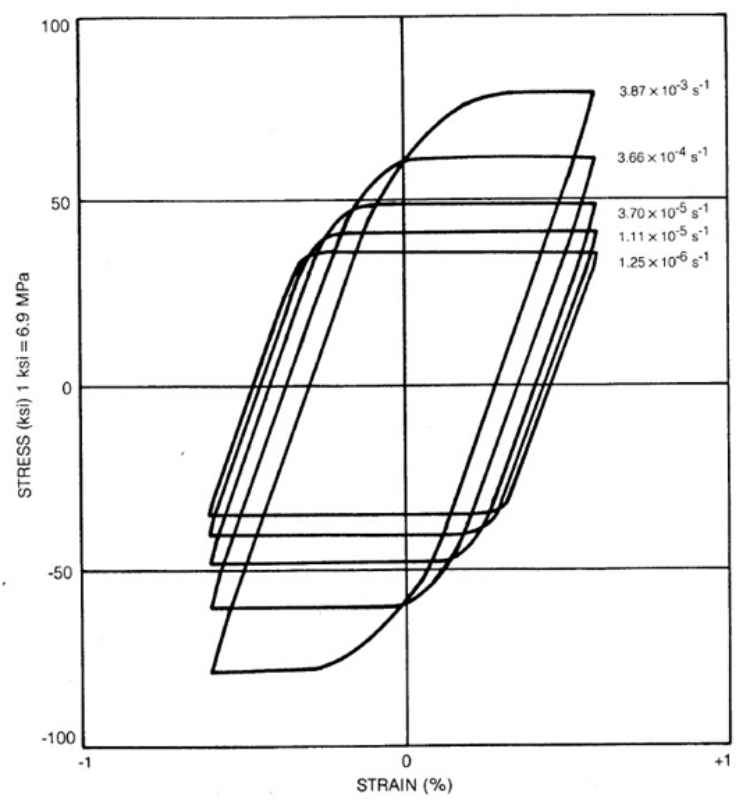

Figure 8: Stress-strain behavior at high $n_{1}$ and $n_{2}$ values

Equation (20) sets the accumulated strain rate $\dot{\varepsilon}^{k}$ equals to the magnitude of the inelastic strain rate $\dot{\varepsilon}^{n}$. The accumulated inelastic strain rate in Walker model is equivalent to the thermal recovery terms on Miller model [57].

$$
\dot{\varepsilon}^{k}=\left|\dot{\varepsilon}^{n}\right|
$$

\subsubsection{MATERIAL CoNSTANTS}

No literature information was found to explain the material constants calculation process. However it is known that Walker model involves cyclic experimental data to determine material constants. Since the constants calculation process is unknown, numerical simulation software is used in the present work to determine material constants for Walker model. 


\subsection{MATERIAL DAMAGE}

Material damage $(\omega)$ refers to a state variable that accounts to the reduction in resistance to failure of the material, caused by loading and extreme environment conditions [58]. Material damage is used as a measure of the material degradation as a function of time. The damage evolutionary state variable considers the material stiffness and strength reductions. Besides material damage determine the damage state within he material bulk and allows life prediction of the material. Usually material damage is divided into two damage terms, creep and fatigue damage. In the present work only creep damage is considered since it will help to predict the tertiary creep regime. The basics of material damage is attributed to Kachanov, who in the 1950 's proposed the foundations of what is now a days known as continuum damage mechanics [59]. Generally, damage is assumed to be homogeneous or continuum throughout the body, thereby the name continuum damage mechanics. Moreover damage is assumed to be irreversible. Damage theory assumed that at an initial time $(t=0)$, the damage state $(\omega)$ is zero, since no force has been applied, and that rupture happens whenever damage $(\omega)$ reaches a unity value $\left(t=t_{r}\right)$. Thus, material damage ranges between zero and unity $\left(0 \leq \omega \leq \omega_{\mathrm{r}}\right.$ where $\left.\omega_{\mathrm{r}}=1\right)$.

\subsubsection{KACHANOV CREEP DAMAGE MODEL}

Kachanov creep damage model uses the term damage to describe the changes in the material due to loading and environment conditions. Kachanov represents damage by using a loss of cross sectional area caused by the growth of internal micro-cracks and micro-voids. As damage increases, the reduction in cross sectional area results in an increase in the internal stress and consequently an increase in the bulk strain. The typical forms of damage that Kachanov investigated were: 
- Transgranular (ductile) fracture, results from the formation of micro-voids within the grains.

- Intergranular (brittle) fracture, results from the accumulation of micro-cracks at the grain boundaries.

- Transgranular and Intergranular (plastic) fracture, results from the formation of micro-voids and micro-cracks as a consequence of large strains.

Equation (21) is the damage rate equation that describes the material damage evolution as a function of the initial stress. The term A and $\mathrm{k}$ represents material constants, while $\omega$ is a state variable that accounts for the damage accumulation.

$$
\dot{\omega}=A\left(\frac{\sigma_{o}}{1-\omega}\right)^{k}
$$

Assuming that at time equal to zero the damage state is zero, since no load is applied and integration of Eq. (21) leads to Eq. (22). Equation (22) allows the determination of the rupture time ( $\left.t_{r}\right)$ based on the material constants $A, k$ and the initial stress $\sigma_{o}$ [59].

$$
t_{r}=\frac{1}{A(1+k) \sigma_{o}^{k}}
$$

Qi et al. [60] determine that the basic theory of critical damage equals to unity is not actually true for Kachanov model. The critical damage value using Kachanov model is less than unity, ranging between $0.2-0.8$ for most metals. Therefore, the damage accuracy of Kachanov model is not granted. Thus, Kachanov model is only considered as background; however, it will not be further considered. 


\subsubsection{SIN-HYPERBOLIC CREEP DAMAGE MODEL}

A recently Sin-hyperbolic based model was developed by Haque and Stewart [61]. This Sin$\mathrm{h}$ model has the capabilities to fully predict the three creep stages. The constitute model equations are the following. Equation (23) represents the damage rate or damage evolution equation which is function of the material constants $M, \phi, \sigma_{t}$, the applied stress $\sigma$, and the state variable $\omega$ which accounts for the damage history of the material. Equation (24) represents the inelastic strain rate evolution equation used by the Sin-h model. Equation (24) consists of the material constant $A$, the stress relationship $\left(\sigma / \sigma_{s}\right)$ from where $\sigma$ is the applied stress and $\sigma_{s}$ is the stress as function of temperature, and the sine hyperbolic function (which gives the name to the model). The term $\lambda$ is not consider a material constants because it can be calculated directly from experimental data by using Eq. (25), which involves the use of the maximum and minimum strain rate of the experimental data. An exponential function (exp) is used in Eq. (23) an Eq. (24) to mitigate the stress sensitivity and mesh dependence issues.

$$
\begin{gathered}
\dot{\omega}=\frac{M[1-\exp (-\phi)]}{\phi} \sinh \left(\frac{\sigma}{\sigma_{t}}\right)^{3} \exp (\phi \omega) \\
\dot{\varepsilon}=A \sinh \left(\frac{\sigma}{\sigma_{s}}\right) \exp \left(\lambda \omega^{3 / 2}\right) \\
\lambda=\ln \left(\frac{\dot{\varepsilon}_{\text {final }}}{\dot{\varepsilon}_{\text {min }}}\right)
\end{gathered}
$$

The previously described equations form a creep-damage constitutive model that accounts for damage and creep. It can observed that damage evolution is predicted only by Eq. (23) and its effects on the inelastic strain rate are described by the exponential (exp) term in Eq. (24). 


\section{CHAPTER 3: MATERIALS}

\subsection{HASTELLOY X}

The creep inelastic behavior of Hastelloy $\mathrm{X}$ is analyzed in the present work. It is a nickelchromium-iron-molybdenum solid-solution-strengthened Ni-base superalloy which possesses an outstanding combination of oxidation resistance and high strength at temperatures of up to $1200^{\circ} \mathrm{C}$, where it exhibits a good ductile behavior. At $1093^{\circ} \mathrm{C}$ the ultimate tensile strength of Hastelloy $\mathrm{X}$ is $97 \mathrm{MPa}$; the Yield Strength at $0.2 \%$ offset is $91 \mathrm{MPa}$. Its melting temperature range is between $1260^{\circ} \mathrm{C}-1355^{\circ} \mathrm{C}[47,62-64]$. Thanks to these mechanical properties Hastelloy $\mathrm{X}$ has been used extensively in the power generation and pressure vessel industry. A large number of studies have been performed on this material characterizing the tensile, rupture, and creep deformation behavior $[64,65]$. The Hastelloy-X experimental data used in the present study comes from Kim et. al.[32]; the alloy used for the experiments was a commercial type hotrolled plate with $19 \mathrm{~mm}$ of thickness. The creep specimens used had a cylindrical form of $30 \mathrm{~mm}$ in gauge length and $6 \mathrm{~mm}$ in diameter. The constant load creep test were conducted at different stress levels $35 \mathrm{MPa}, 30 \mathrm{MPa}, 25 \mathrm{MPa}, 20 \mathrm{MPa}, 18 \mathrm{MPa}, 16 \mathrm{MPa}$, and $14 \mathrm{MPa}$ at $950{ }^{\circ} \mathrm{C}$. At $950^{\circ} \mathrm{C}$ Hastelloy $\mathrm{X}$ exhibited an average young modulus of $144 \mathrm{GPa}$, a poison's ratio of 0.29 and a $0.2 \%$ yield strength of $121 \mathrm{MPa}$. The nominal chemical composition of the Hastelloy $\mathrm{X}$ is provided in Table 1.

Table 1: Hastelloy X chemical composition (weight. \%) [32]

\begin{tabular}{cccccccc}
\hline $\mathbf{N i}$ & $\mathbf{C}$ & $\mathbf{M n}$ & $\mathbf{S i}$ & $\mathbf{C r}$ & $\mathbf{C o}$ & $\mathbf{C u}$ & Mo \\
\hline 48.04 & 0.082 & 0.82 & 0.42 & 21.91 & 0.79 & 0.13 & 8.65 \\
\hline $\mathbf{W}$ & $\mathbf{F e}$ & $\mathbf{B}$ & $\mathbf{A l}$ & $\mathbf{T i}$ & $\mathbf{P}$ & $\mathbf{N}$ & $\mathbf{S}$ \\
\hline 0.44 & 19.0 & 0.002 & 0.17 & 0.007 & 0.013 & 0.015 & 0.0003 \\
\hline
\end{tabular}




\subsection{STAINLESS STEEL 304}

The second considered material is stainless steel 304, an austenitic iron-nickel-chromium alloy that possesses high strength and high resistance at elevated temperatures where it exhibits a ductile behavior. At $800^{\circ} \mathrm{C}$ the ultimate tensile strength of stainless steel 304 is $135 \mathrm{MPa}$; the yield strength at $0.2 \%$ offset is $70 \mathrm{MPa}[66]$. The melting temperature range is $1399^{\circ} \mathrm{C}-1454{ }^{\circ} \mathrm{C}$ [67]. Thus, thanks to these mechanical properties and its repeated use on piping applications, this material is considered in the present study.

The stainless steel 304 experimental data used in the present work was found in Stewart $\mathrm{Ph}$., D. dissertation work [68]. The specimens used during the fatigue testing were rod annealed and cold finished to improve strength and straightness. Low cycles fatigue test were conducted at $600^{\circ} \mathrm{C}$ and at $0.5 \%$ and $0.7 \%$ strain amplitude $(\Delta \varepsilon)$. The tested material was prepared to meet ASTM standards A276 and A479, the nominal chemical composition of stainless steel 304 is provided Table 2 .

Table 2: stainless steel 304 chemical composition (weight. \%) [68]

\begin{tabular}{cccccc}
\hline Fe & Cr & Ni & C & Mn & Cu \\
\hline 69.0 & 19.0 & 9.25 & 0.04 & 1.0 & 0.5 \\
\hline Mo & $\mathbf{S i}$ & $\mathbf{S}$ & $\mathbf{P}$ & $\mathbf{C o}$ & $\mathbf{N}$ \\
\hline 0.5 & 0.5 & 0.015 & 0.023 & 0.1 & 0.05 \\
\hline
\end{tabular}




\section{CHAPTER 4: NUMERICAL OPTIMIZATION SOFTWARE}

The numerical optimization software used in the present work to calculate Miller and Walker material constants is the MAterial Constant Heuristic Optimizer (MACHO) software. This new FORTRAN based software was recently developed at The University of Texas at El Paso to optimize material constants of complex constitutive models. This software allows the optimization of material constants by comparing experimental data to multiple iterations of simulated mechanical test results, until convergence parameters are meet. Besides MACHO calculates an objective function value which reflects the goodness of fit between the simulated mechanical test results and the experimental data. The objective function value is useful because it gives a good estimation of which constants set possess a higher accuracy. The provided experimental data and boundary conditions play a fundamental role in the optimization process. In the case of comparing viscoplasticity models' performance such as in the present work, the use of MACHO is extremely helpful because it ensures a systematic calculation of material constants. Using the same optimization parameters for both models, a fair comparison between the models can be establish since the material constants for both models were calculated/optimized under the same conditions. MACHO is based on the simulated annealing algorithm; within this algorithm, an initial temperature is given and the model evaluation at the given temperature takes place until a given number of iterations are reached or convergence parameters are reached. Afterward, a temperature reduction is executed and function evaluation at this new temperature again takes place; this process is repeated until convergence is reached. 


\subsection{OPTIMIZATION PROCESS}

The optimization process is illustrated in Figure 9. It is as follows: initial guess constants, boundary conditions and experimental data must be provided by the user. These initial guess constants should preferably fit experimental data closely to allow the optimization algorithm to calculate constants within a more promising area. To determine initial guess values, both constitutive models were programmed in spreadsheets where experimental data was plotted and different material constants values from literature were modified until material constants values capable of fitting experimental data closely were found. The experimental data and boundary conditions files must contain the time-stress and time-strain relation of the physical experiment. The time relation must be the exactly the same between the experimental data and the boundary condition file. Depending on the mechanical test type the experimental data and boundary condition files would be different. For a load control test the experimental data file will contain the time-strain data, while the boundary condition file will contain the time-stress data. On the other hand, for a displacement control test the experimental data file will contain time-stress data while the boundary condition file will contain time-strain information.

MACHO possess the capabilities to optimize material constants considering $n$ number of experimental data sets at the same time. Besides a combination of load controlled and displacement controlled data sets can be simultaneously optimized; however, the combination of experimental data types might lead to the calculation of less optimal material constants when compared to the use of only one type of experimental data. The experimental data and boundary conditions are characteristic of each material; therefore, only one material can be optimized at a time. In the present work the material constants for Hastelloy X creep (load controlled) were 
calculated apart from the material constants for stainless steel 304 low cycle fatigue (displacement controlled).

In the next step the Finite Element (FE) simulation of the desired mechanical test takes place by using the initial guess constants and the programmed constitutive model equations. The approach used to perform the FE simulation is executed based on the mechanical test type, load controlled or displacement controlled. For load control tests the process is simple, because the applied stress is directly used in the constitutive model equations to calculate an updated inelastic strain rate. On the other hand, if the test is displacement controlled, then radial return mapping using Newton-Raphson iteration takes place to solve for an updated stress which will then be used to calculate an updated inelastic strain rate.

Before the calculation of the objective function value (which indicates the goodness of fit of the simulation results with respect to experimental) takes place, a linear interpolation of the simulation results is perform. This linear interpolation sets the simulation results time step size equal to the experimental data's; therefore, allowing a comparison between the experimental data and the simulated data at exactly the same time step. Then the objective function value is calculated based on the type of test; for load controlled test Tertiary Creep Function algorithm is used, while Low Cycle Fatigue Function algorithm is used for displacement controlled. Both methods calculate the normalized least squares values. The reason of having two methods is because the objective function value of two types of test is not directly comparable, since the weight influence (or importance) of each data set might vary due to the duration of the test or the length of the experimental data set. The objective value of each data set is summed into one objective function value, which is represented by the sum of least squares. Afterwards, the objective value is compared to convergence parameters, if convergence has not been reached, 
then the simulated annealing optimization algorithm is executed. The simulated annealing algorithm is based on the metallurgical method of annealing, where a material heated above its recrystallization temperature is cooled at a low cooling rate to reach a low energy state. The smaller the cooling rate the lower the energy state the material will result in. Based on this method, the simulated annealing algorithm accepts low cooling rates (low temperature reduction rates) to control the probability of accepting worse solutions. With a low cooling temperature rate this probability is reduced, resulting in more precise function evaluations. The simulated annealing algorithm suggests that slowing the temperature reduction will result in lower global minima $[69,70]$. Thereafter, the algorithm will produce a new set of material constants which are used to start the process all over again until convergence is reached. 


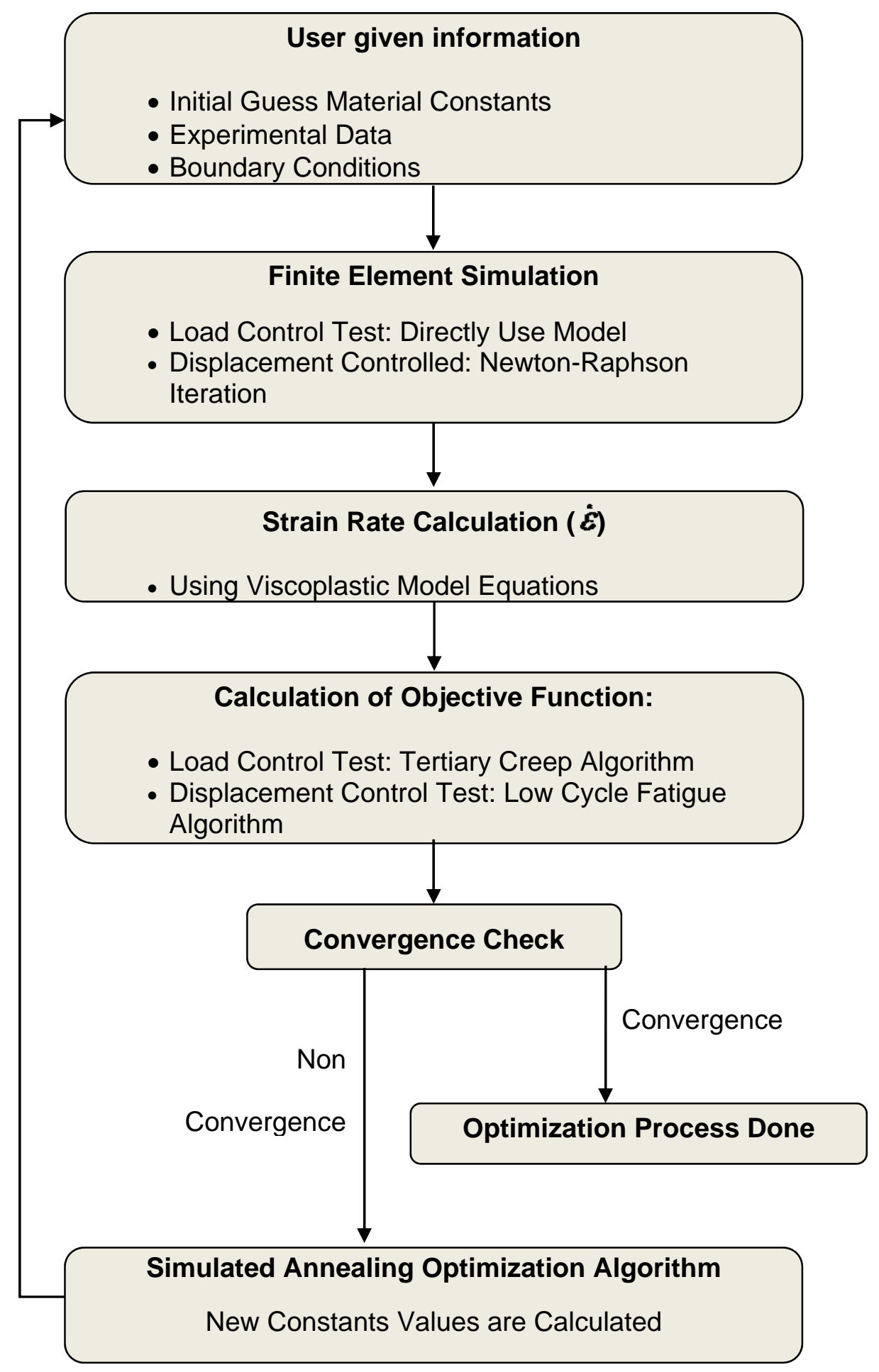

Figure 9: MACHO material constant optimization process 


\subsection{OPTIMIZATION PARAMETERS}

MACHO allows the user to establish critical optimization parameters that control the optimization software performance to obtain better results. Some controlling parameters of the simulated annealing algorithm are initial temperature $\mathrm{Ti}$, temperature reduction factor $\mathrm{rT}$, total number of steps Ns at each temperature level before cutting temperature, convergence controls within a temperature level, and maximum number of iterations. A high enough initial temperature will allow the algorithm to evaluate the gross behavior of the model since the algorithm is performing uphill movements finding optimal constants in the global surface. A low initial temperature will reduce the model's evaluation in a global surface, therefore not ensuring a global evaluation of constants values. The temperature reduction factor should be low enough to keep a low cooling rate of the annealing simulation. The maximum number of iterations is dependent on the number of optimized material constants of the model, high number of optimized material constants will require a high number of iterations since its optimization will take longer. Other parameters that can be adjusted on the software are the total number of constants to optimize N, set upper and/or lower boundaries for the optimized constants values; this contributes to a faster and more accurate optimization of constants since the possible solutions are narrowed to a smaller set of possibilities. The regulation of these parameters, control the duration and accuracy of the optimization process. 


\section{CHAPTER 5: EXERCISE OF MILLER AND WALKER MODELS}

\subsection{OPTIMIZED MATERIAL CONSTANTS}

Table 3 and Table 4 show the initial guess material constants values used by MACHO during the optimization process for Hastelloy $\mathrm{X}$, and the resultant optimized material constants values, which were used for the simulation and exercise of both constitutive models to predict Hastelloy $\mathrm{X}$ behavior under creep. The same experimental data sets and optimization parameters were used for both models during their optimization process; it consisted of 500,000 iterations, with a temperature reduction factor of 0.5 and $100^{\circ} \mathrm{C}$ as the initial temperature. As mentioned before, the objective function value is a measure of how good the optimized material constants fit the experimental data (it is unit less).

Figure 10 shows the resultant objective function values with respect to the iteration number during the material constants optimizing process of the Miller and Walker constitutive models for Hastelloy $\mathrm{X}$ under creep. It can be observed how during the first iterations the objective function values exhibited a large oscillation for both constitutive models; these oscillations are the result of the non-convergence of the optimization process due to the use of non-proper material constants values. Similarly, it can be observed how after several thousand iterations both models reach convergence resulting in a constant objective function value which in the figure is described by a constant horizontal line. Likewise, according to the figure the Miller model reach convergence at an earlier iteration number, while the Walker model reaches convergence at a later stage. The convergence difference is attributed to the number of material constants being optimized for each constitutive model, where seven material constants were optimized for Miller model and nine for Walker model. The objective function values for each constitutive model are shown in Table 3 and Table 4. 


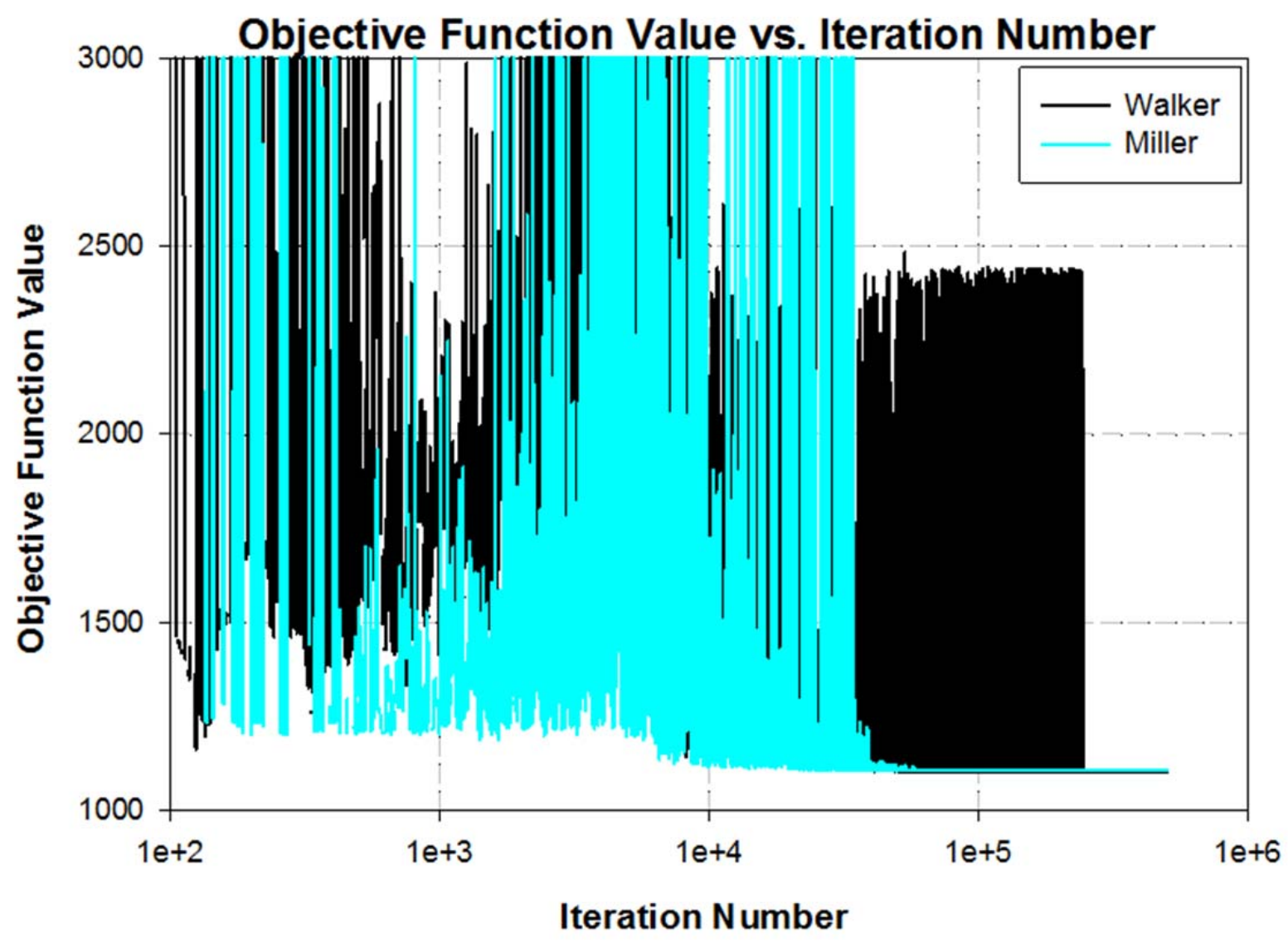

Figure 10: Objective Function results of the optimization process of Miller and Walker models for Hastelloy X under creep.

For the initial guess constants values the objective function difference between Miller and Walker model is $3.04 \%$, whereas for the optimized constants values the objective function difference between Miller and Walker is only $0.4 \%$. Thus, both models' constants were optimized under the same conditions and their goodness of fit or objective function value is reasonably similar for both models, ensuring a systematic calculation of material constants for both constitutive models. According to Walker $[56,57]$ only nine material constants are required to model creep, Table 4 shows the nine material constants used to exercise the Walker model. In an effort to optimize all fourteen material constants, optimization simulations were performed, resulting in higher objective function values; thus, demonstrating that not all material constants 
of Walker model are required to model creep. These zero values will deactivate some functions or terms of the constitutive equations, especially the recovery terms of the model; resulting effects are discussed in the next section.

Walker model material constants were compared values found in literature [56] for Hastelloy $\mathrm{X}$ and the following differences were determined. The optimized value of constant $B$ is smaller compared to that found in literature; this indicates that all the optimized values will result in a high magnitude of inelastic strain rate. Therefore a small $B$ value is required to lower the inelastic strain value and tune it to the proper range. None significant difference has been found in the $n$ material constant values. The material constant $K_{l}$ is smaller in the optimized values and since the drag stress equation is a non-evolutionary equation (constant value) it means that the optimized values are determining a smaller constant drag stress value compared to literature values. The optimized value of $n_{6}$ is larger than that of literature, this difference will be affecting the recovery rate equation by causing a smaller recovery magnitude in the rest stress. The optimized value of $n_{3}$ is a smaller value compared to literature values, this difference will affect the dynamic recovery term of the rest stress by causing a lower magnitude of rest stress recovery. So the optimized values magnitude difference of constant $n_{6}$ and $n_{3}$ in the optimized values compensates the value difference between these constants in literature values. The Miller model optimized material constant values cannot be compared to literature, because no material constant values have been found for Hastelloy X.

Table 3: Miller model initial guess and optimized material constants for Hastelloy X creep

\begin{tabular}{cccc}
\hline $\begin{array}{c}\text { Material } \\
\text { constant }\end{array}$ & $\begin{array}{c}\text { Initial guess } \\
\text { value }\end{array}$ & $\begin{array}{c}\text { Optimized } \\
\text { value }\end{array}$ & Units \\
\hline$B$ & $0.45107 \mathrm{e} 14$ & $0.41229 \mathrm{e} 13$ & $\mathrm{Sec}^{-1}$ \\
\hline
\end{tabular}




\begin{tabular}{cccc}
\hline$n$ & 3.2003 & 3.2608 & - \\
\hline$H_{1}$ & $0.32995 \mathrm{e}-4$ & $0.14383 \mathrm{e}-3$ & $\mathrm{MPa}$ \\
\hline$H_{2}$ & $0.21839 \mathrm{e} 6$ & $0.33919 \mathrm{e} 7$ & - \\
\hline$A_{1}$ & 11.351 & 7.0297 & $\mathrm{MPa}^{-1}$ \\
\hline$A_{2}$ & $0.12418 \mathrm{e}-13$ & $0.17724 \mathrm{e}-14$ & $\mathrm{MPa}^{-1}$ \\
\hline$C_{2}$ & $0.12030 \mathrm{e}-27$ & & $\mathrm{MPa}$ \\
\hline Obj. Funct. & $1,290.215$ & $1,106.046$ & - \\
\hline
\end{tabular}

Table 4: Walker model initial guess and optimized material constants for Hastelloy X creep

\begin{tabular}{cccc}
\hline $\begin{array}{c}\text { Material } \\
\text { constant }\end{array}$ & $\begin{array}{c}\text { Initial guess } \\
\text { value }\end{array}$ & $\begin{array}{c}\text { Optimized } \\
\text { value }\end{array}$ & Units \\
\hline$B$ & $1.46 \mathrm{e}-7$ & $.13545 \mathrm{e}-6$ & - \\
\hline$K_{1}$ & 4.5870 & 4.5183 & $\mathrm{MPa}$ \\
\hline$K_{2}$ & 0 & 0 & - \\
\hline$R_{o}$ & 15 & 15 & $\mathrm{MPa}$ \\
\hline$R_{S}$ & 7.3055 & 8.3276 & - \\
\hline$m$ & 6.3459 & 6.3480 & - \\
\hline$n$ & 4.8584 & 4.8582 & - \\
\hline$n_{1}$ & 0 & 0 & - \\
\hline$n_{2}$ & -3.4 & -3.4 & $\mathrm{MPa}$ \\
\hline$n_{3}$ & -2 & -2 & - \\
\hline$n_{4}$ & 0 & 0 & - \\
\hline$n_{5}$ & 0 & 0 & - \\
\hline$n_{6}$ & $1 \mathrm{e}-8$ & -.27462 & $\mathrm{Sec}^{-1}$ \\
\hline$n_{7}$ & 0 & 0 & - \\
\hline Obj. Funct. & $1,251.04$ & $1,101.63$ & - \\
\hline
\end{tabular}

Two different alloys are considered in the present work to test the flexibility and capabilities of the considered constitutive models and MACHO algorithm. Stainless steel 304 was used to test the modeling capabilities at low cycle fatigue conditions. Different optimization parameters were used for low cycle fatigue optimization process to perform a better optimization of material 
constants. 500,000 iterations were conducted at an initial temperature of $125^{\circ} \mathrm{C}$ and a temperature reduction factor of 0.25 . Table 5 and Table 6 show the initial guess material constants used by MACHO during the optimization process and the optimized material constants for stainless steel 304, along with the objective function value for each set of constants.

The difference in objective function value at initial guess constants values between Miller and Walker for stainless steel 304 constants is $14.32 \%$; whereas for the optimized material constants the objective function value difference is $46.40 \%$. Even though the final objective functions value for low cycle fatigue is not as close as in creep for between both models, it still allows a comparison between the models since both models were optimized under the same conditions. This difference reflects the capability difference between both models to predict low cycle fatigue. The fatigue optimization objective function magnitudes are not comparable to creep's because the area under the creep curves are not of the same magnitude as the fatigue curves.

Miller model optimized material constant values were compared to values found in literature [53] for 304 stainless steel and the following differences were determined. The optimized material constant $B$ has a lower value than in literature, this difference means that all the optimized materials constants will predict an overall higher inelastic strain magnitude compared to literature material constants. Therefore a high tuning value for constants $B$ is not required. The material constant $n$ does not present a considerable difference between the optimized and literature values; however the optimized value is small, meaning that the stress intensity calculated by the optimized values is higher. The optimized constant values for $H_{1}$ and $A_{1}$ are smaller than those in literature; these differences indicate that the rest stress will be evolving in lower magnitudes compared to using the literature values. On the other hand, the optimized 
material constants $A_{2}$ and $C_{2}$ present a higher value compared to literature values. This difference indicates that the drag stress will be evolving at a higher magnitude compared to using the literature values. So the overall difference in material constant values between optimized and literature values, indicates that by using the optimized values the drag stress will be calculated in higher magnitudes, and this increase will be compensated by a lower calculation of rest stress. The optimized material constants for Walker model cannot be compared to literature, because no material constants have been found in literature for 304 stainless steel.

Figure 11 shows the number of iterations with respect to the objective function value for each model during low cycle fatigue optimization process. It can be observed how the Walker model exhibited a larger variation on the objective function value compared to Miller model. Miller model reached convergence at an earlier stage compared to Walker model; however, this was expected because it contains fewer variables. Considering the final objective function value the Walker model resulted in a smaller value compared to Miller model, this is attributed to the higher capabilities the model possess and they are reflected in the larger number of necessary material constants. 


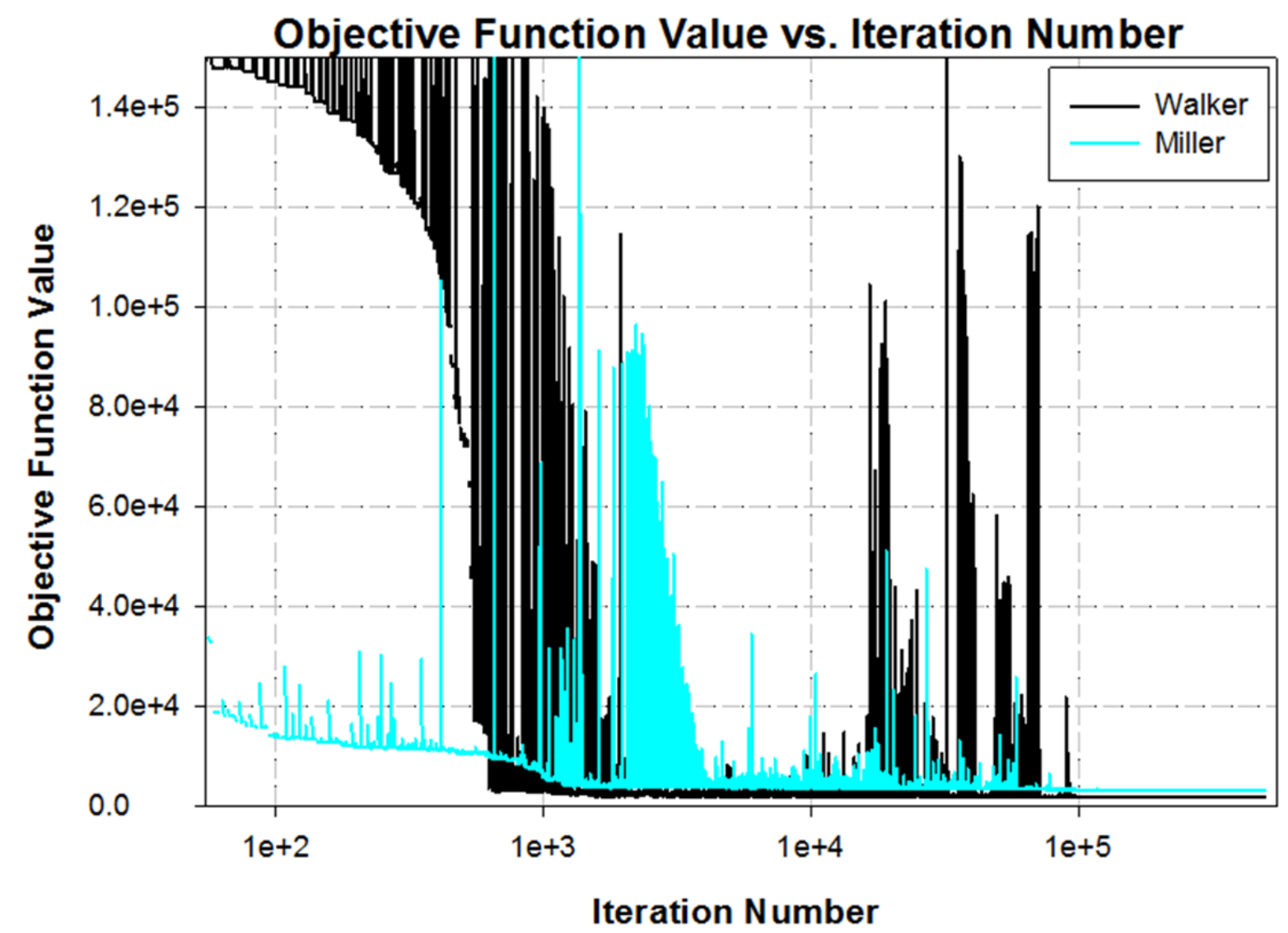

Figure 11: Objective function results of the optimization process of Miller and Walker models for 304 stainless steel under fatigue

According to Walker $[56,57]$ nine material constants are required to be active to model low cycle fatigue. Optimization simulations were conducted for nine material constants (as in creep) and for fourteen material. The results showed that the use of nine material constants resulted in a better correlation between simulated and experimental data by more than $90 \%$ compared to fourteen constants. Therefore only nine material constants were used to exercise the Walker model. 
Table 5: Miller model initial guess and optimized material constants for stainless steel 304

\begin{tabular}{cccc}
\hline $\begin{array}{c}\text { Material } \\
\text { constant }\end{array}$ & $\begin{array}{c}\text { Initial guess } \\
\text { value }\end{array}$ & $\begin{array}{c}\text { Optimized } \\
\text { value }\end{array}$ & Units \\
\hline$B$ & $0.10 \mathrm{e}-14$ & $0.56864 \mathrm{e}-4$ & $\mathrm{Sec}^{-1}$ \\
\hline$n$ & 5.8 & 1.2661 & - \\
\hline$H_{1}$ & 100.0 & $0.41389 \mathrm{e}-3$ & $\mathrm{MPa}$ \\
\hline$H_{2}$ & 280.0 & 9705.50 & - \\
\hline$A_{1}$ & $0.7420 \mathrm{e}-4$ & $0.2407 \mathrm{e}-5$ & $\mathrm{MPa}^{-1}$ \\
\hline$A_{2}$ & 0.800 & $0.41394 \mathrm{e}-1$ & $\mathrm{MPa}^{-1}$ \\
\hline$C_{2}$ & 0.100 & $0.15996 \mathrm{e} 9$ & $\mathrm{MPa}^{-}$ \\
\hline Obj. Funct. & $129,831.37$ & $3,292.2774$ & - \\
\hline
\end{tabular}

Table 6: Walker model initial guess and optimized material constants for stainless steel 304

\begin{tabular}{cccc}
\hline $\begin{array}{c}\text { Material } \\
\text { constant }\end{array}$ & $\begin{array}{c}\text { Initial guess } \\
\text { value }\end{array}$ & $\begin{array}{c}\text { Optimized } \\
\text { value }\end{array}$ & Units \\
\hline$B$ & $0.77186 \mathrm{e}-5$ & $0.41691 \mathrm{e}-3$ & - \\
\hline$K_{1}$ & 27.386 & 169.16 & $\mathrm{MPa}$ \\
\hline$K_{2}$ & 0 & 0 & - \\
\hline$R_{o}$ & 2.22 & 14.696 & $\mathrm{MPa}$ \\
\hline$R_{s}$ & $0.8181 \mathrm{e} 20$ & $0.18133 \mathrm{e} 20$ & - \\
\hline$m$ & $0.52505 \mathrm{e} 20$ & $0.78026 \mathrm{e} 19$ & - \\
\hline$n$ & 22.0 & 7.8255 & - \\
\hline$n_{1}$ & 0 & 0 & - \\
\hline$n_{2}$ & $6,954.1$ & 24,252 & - \\
\hline$n_{3}$ & 182.89 & 279.18 & - \\
\hline$n_{4}$ & 0 & 0 & - \\
\hline$n_{5}$ & 0 & 0 & $\mathrm{Sec}^{-1}$ \\
\hline$n_{6}$ & $0.94574 \mathrm{e} 10$ & $0.13881 \mathrm{e} 20$ & - \\
\hline$n_{7}$ & 0 & 0 & - \\
\hline Obj. Funct. & $151,525.35$ & $1,764.814$ & \\
\hline
\end{tabular}




\subsection{RESULTS}

Miller and Walker constitutive models were exercised in ANSYS Mechanical APDL using UPFs to program the constitutive equations and input decks to code simulation parameters and boundary conditions. The models were exercised to model the creep behavior of Hastelloy X at a constant temperature of $950^{\circ} \mathrm{C}$ at different stress levels $35 \mathrm{MPa}, 30 \mathrm{MPa}, 25 \mathrm{MPa}, 18 \mathrm{MPa}, 16$ $\mathrm{MPa}$ and $14 \mathrm{MPa}$, and to model the fatigue behavior of stainless steel 304 at a constant temperature of $600^{\circ} \mathrm{C}$ at $0.5 \%$ and $0.7 \%$ strain amplitude $(\Delta \varepsilon)$. MACHO optimized material constants were used for these numerical simulations. Figure 12 - Figure 14 show the creep numerical simulation results of Miller model, compared to Hastelloy X experimental data. Miller model behavior under creep conditions is presented and compared to experimental data in Figure 12. It can be observed that even though this model was design to account only for primary and secondary creep, when good optimized material constants values are used the model gains the ability to accurately model tertiary creep at high stress levels. On the other hand, at low stress levels the model is able to predict only primary and secondary creep as it is designed to. As shown in the figure, as the applied stress level decreases the Miller model becomes less accurate. Good correspondence between experimental data and numerical simulation results is observed for stresses $\sigma \geq 25 \mathrm{MPa}$, where full creep is predicted. On the other hand, scatter is present between experimental data and numerical simulation results at $\sigma<25 \mathrm{MPa}$. At these stress levels, primary and secondary creep is predicted with some discrepancies; thus, conservative consideration of the material capabilities at low stresses would result.

The drag stress or isotropic hardening behavior of the Miller model during numerical simulations is shown in Figure 13. The drag stress describes the stress impeding dislocation motion caused by grain boundaries, solute atoms, and precipitate atoms. Simulation results show 
the drag stress linearly decreasing with time; physically meaning that as time increases dislocation motion increases due to the climbing and slip of dislocations. At high stresses the drag stress reduction will be larger and faster, meaning that the higher the applied stress, the less time it will take to have a drag stress or isotropic hardening reduction, since there is more applied stress involved to overcome obstacles impeding dislocation motion . On the contrary, at low applied stresses, the reduction of drag stress is smaller and slower, meaning that solute atoms and grain boundaries prevent dislocation motion for a longer period of time and allowing only a slight motion. Figure 14 shows Miller's rest stress or kinematic hardening behavior during numerical simulations of creep. The rest stress represents the amount of stress that stuck dislocations are causing within the lattice due to the applied stress. As presented in the figure, as time increases, so does the rest stress, meaning that as time increases more stress is generated by the stuck dislocations, thus affecting the stress concentration within the lattice. At high stress levels high rest stress are generated, whereas at low stress levels lower rest stresses are generated. This is due to the fact that the opposing or back stress is proportional to the applied stress.

Figure 15 - Figure 17 show the creep numerical simulation results of Walker model, compared to Hastelloy X experimental data. Walker's model also gains the ability to describe the tertiary creep regime of Hastelloy $\mathrm{X}$ given optimized material constants, even though it is not included in its original designed capabilities. Figure 15 presents Walker model behavior under creep and compares it to experimental data. Walker model exhibits high precision predicting creep at high stress levels where the three creep stages are predicted. However, at low stress levels, the constitutive model meets its design purposes and describes the primary and secondary creep regime. Thus, as the stress level decreases the modeling accuracy decreases. As it is shown 
in the figure, good correspondence between the numerical simulation results and experimental data is observed at $\sigma \geq 20 \mathrm{MPa}$. On the contrary, scatter between the same parameters is present at $\sigma<20 \mathrm{MPa}$. The unusual behavior of Walker model predicting tertiary creep at high stress levels is attributed to the optimized material constants used for the numerical simulations. Drag stress behavior during Walker model numerical simulation is presented in Figure 16. The drag stress behavior is represented by a straight line for all stress levels. This is expected since the drag stress equation is a non-evolutionary drag stress equation governed only by the material constant $k_{l}$, which has a constant value throughout the simulation. The remaining terms of the drag stress equation were deactivated because they were not required to describe creep phenomena according to Walker and Hartmann [56, 57]. Figure 17 shows the rest stress behavior during the simulation of Walker model. As presented in the figure, as time increases the rest stress decreases. However, the rest stress reduction is dependent on the stress level. At low stress levels the rest stress reduction is slow and short, whereas at high stress levels the drag stress reduction is fast and larger. This difference in rest stress reduction is due to the test time and applied stresses. The rest stress value is decreasing because of the deactivation of constant $n_{l}$ in the rest stress rate equation. Constant $n_{l}$ is affecting the hardening and recovery terms of the equation where it is affected by the inelastic strain rate; however, in the recovery term it is also function of the recovery relation rate which is an increasing number based on the accumulated inelastic strain rate equation. Thus, the deactivation of the material constant $n_{1}$ is leading to a reduction in the rest stress behavior.

Comparing the drag stress evolution of the two exercised constitutive models, it can be concluded that the Walker model drag stress equation is a non-evolutionary equation governed by a constant value $k_{1}$. On the other hand, Miller model uses an evolutionary drag stress equation 
dependent on multiple material constants and strain rates responsible of the drag stress value evolution. Comparing the rest stress evolution behavior of the two considered viscoplastic models, it can be concluded that Walker softening terms in the rest stress rate equations have a higher influence than in Miller model. This behavior is attributed to the optimized and deactivated material constants. In terms of the magnitude values of the drag and rest stress of both models, the differences are attributed to the different mathematical functions present in the inelastic strain rate equations. Walker model uses only a power law function; whereas Miller model uses a hyperbolic sine function and two power law functions. Therefore in order to balance the difference of these mathematical functions, the drag and rest stress magnitudes must be different.

The behavior of the rest and drag stress for the Walker model during these numerical simulations is unrealistic, since the behavior does not correspond to the actual physics present during a physical test. This unrealistic behavior of Walker rest and drag stress is attributed to the optimized material constants, which were optimized to fit the numerical simulations to the experimental data and not to follow the actual physics of the phenomena. This illustrates that MACHO is agnostic to the physics of the material behavior; it finds the optimal material constants that would best fit the experimental data by following a mathematical algorithm. That is the reason why both constitutive models gained the ability to model tertiary creep. Even though Miller model drag and rest stress behavior matched the actual physics of the deformation mechanisms, no literature information was found to support the precision of the magnitudes of these parameters with respect to the numerical simulations results. Therefore, a laboratory experiment is recommended to experimentally determine the real values of Hastelloy $\mathrm{X}$ rest and drag stress. 


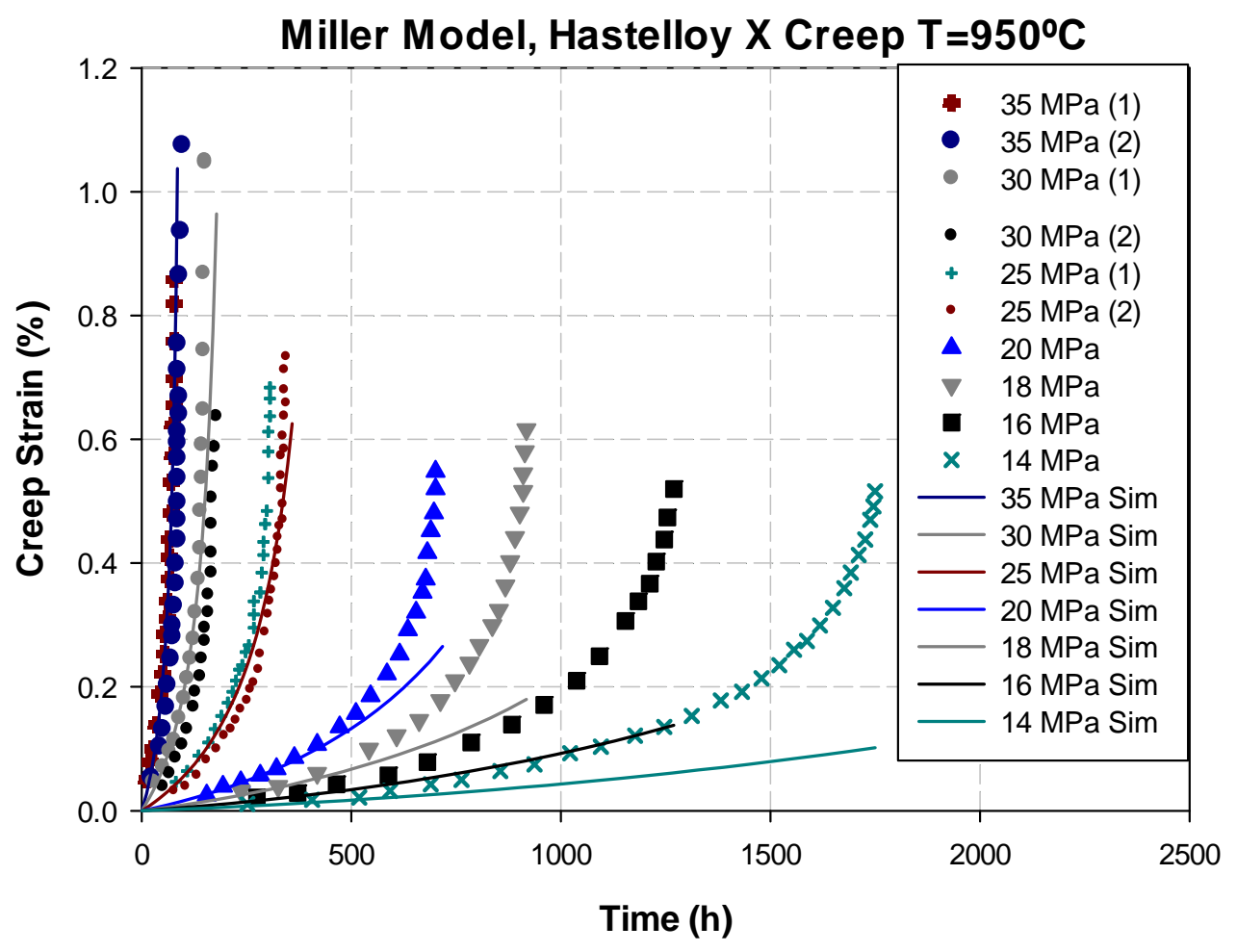

Figure 12: Miller model simulation vs. experimental data [32]

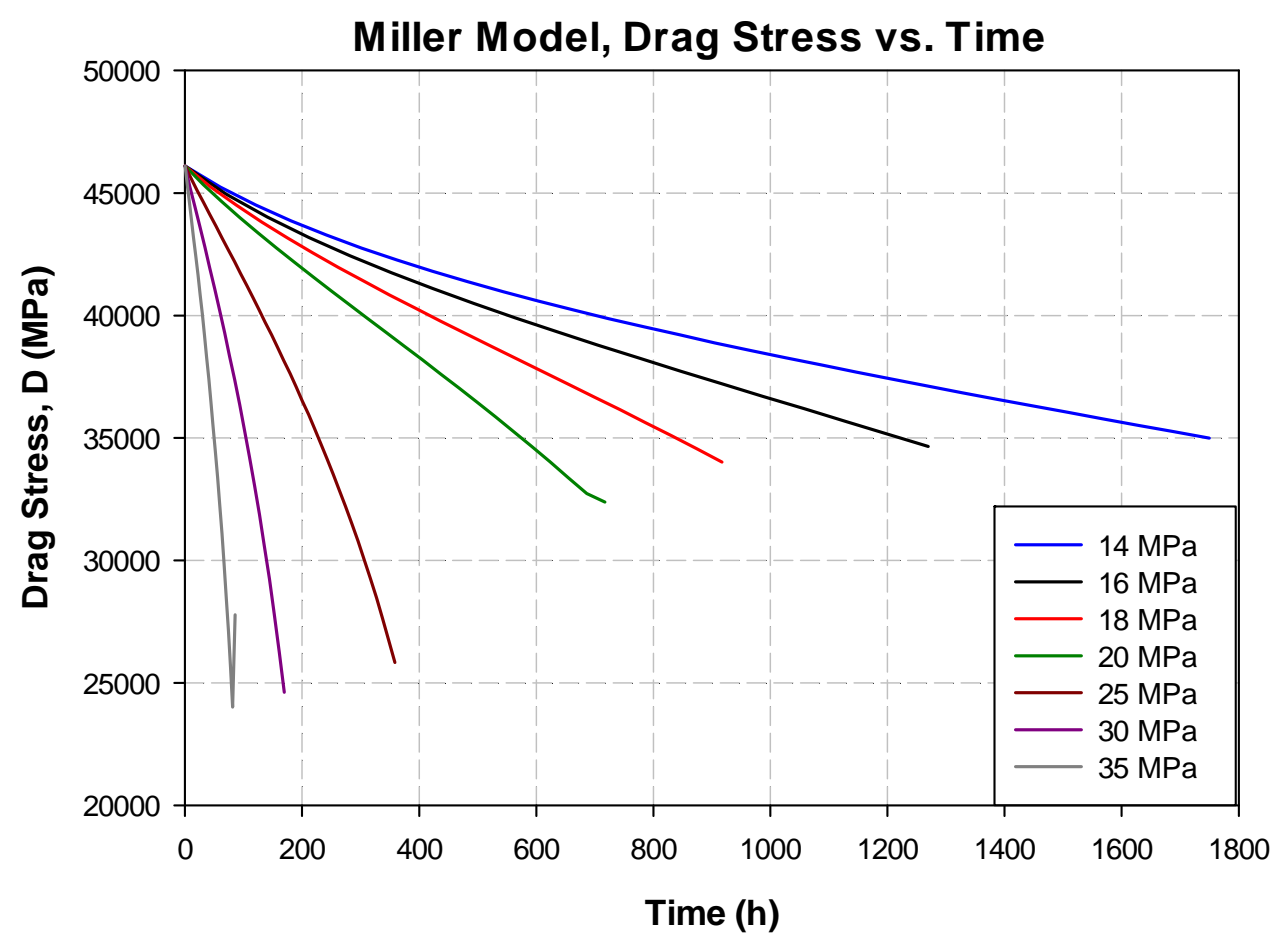

Figure 13: Miller model drag stress vs. time 


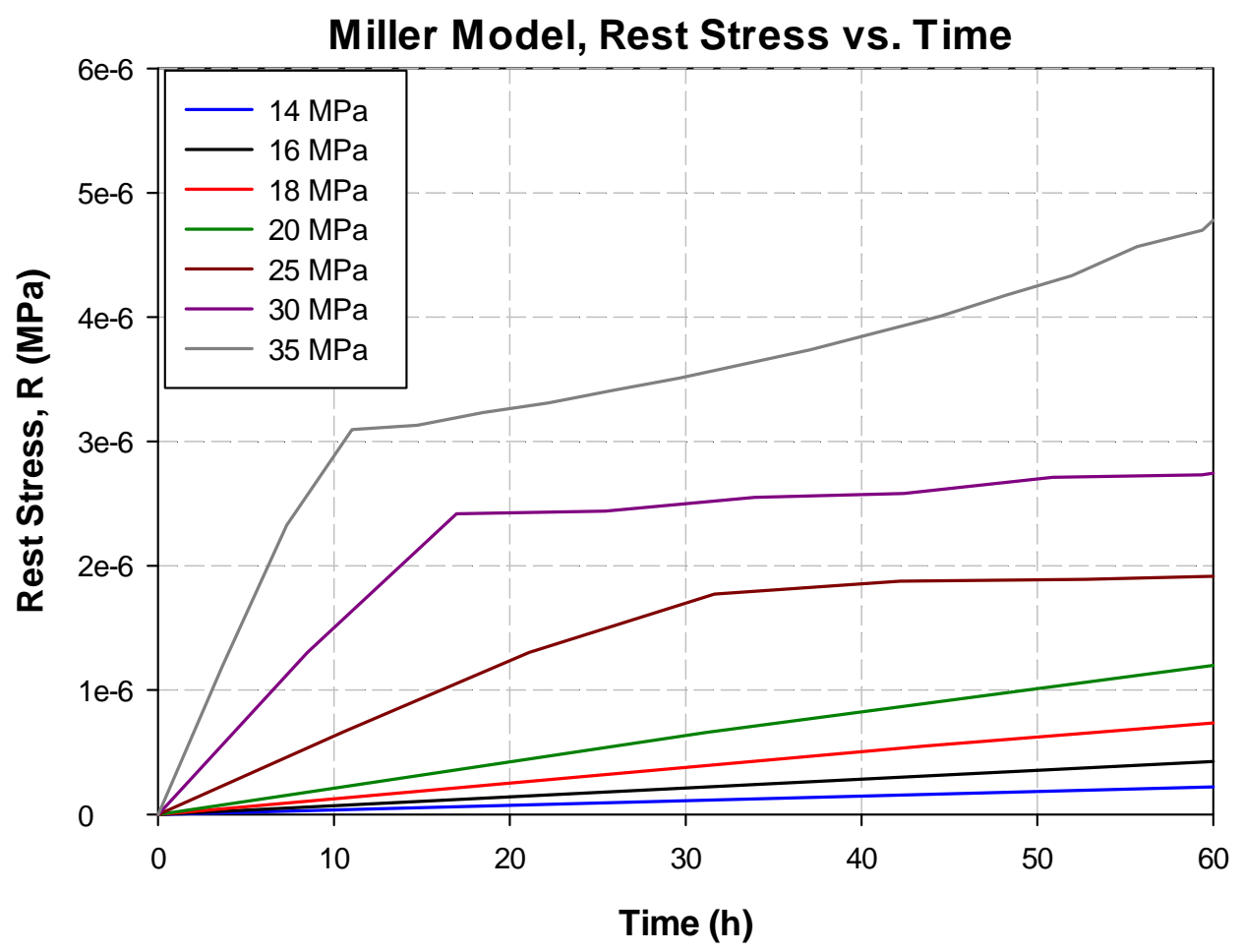

Figure 14: Miller model rest stress vs. time

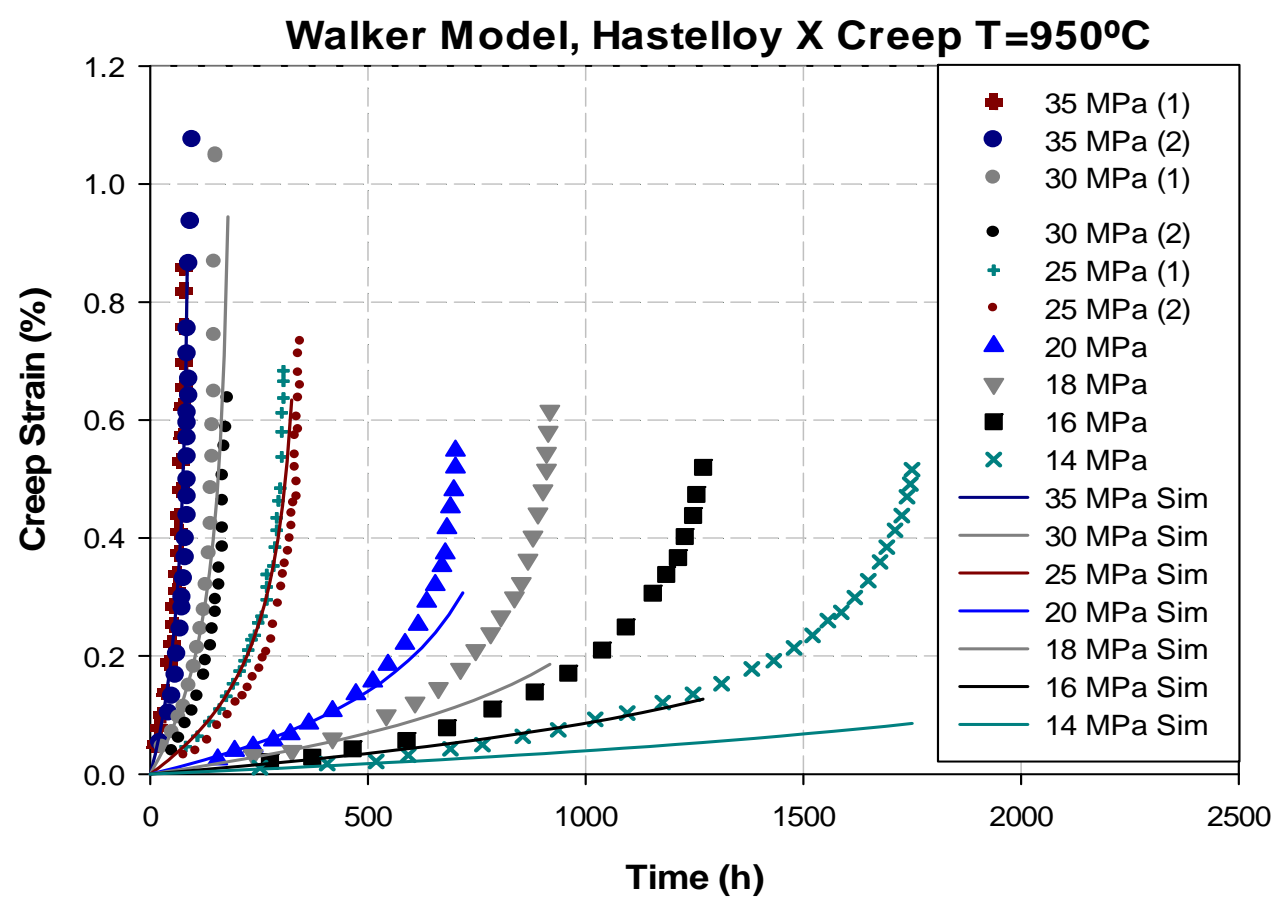

Figure 15: Walker model simulation vs. experimental data [32] 


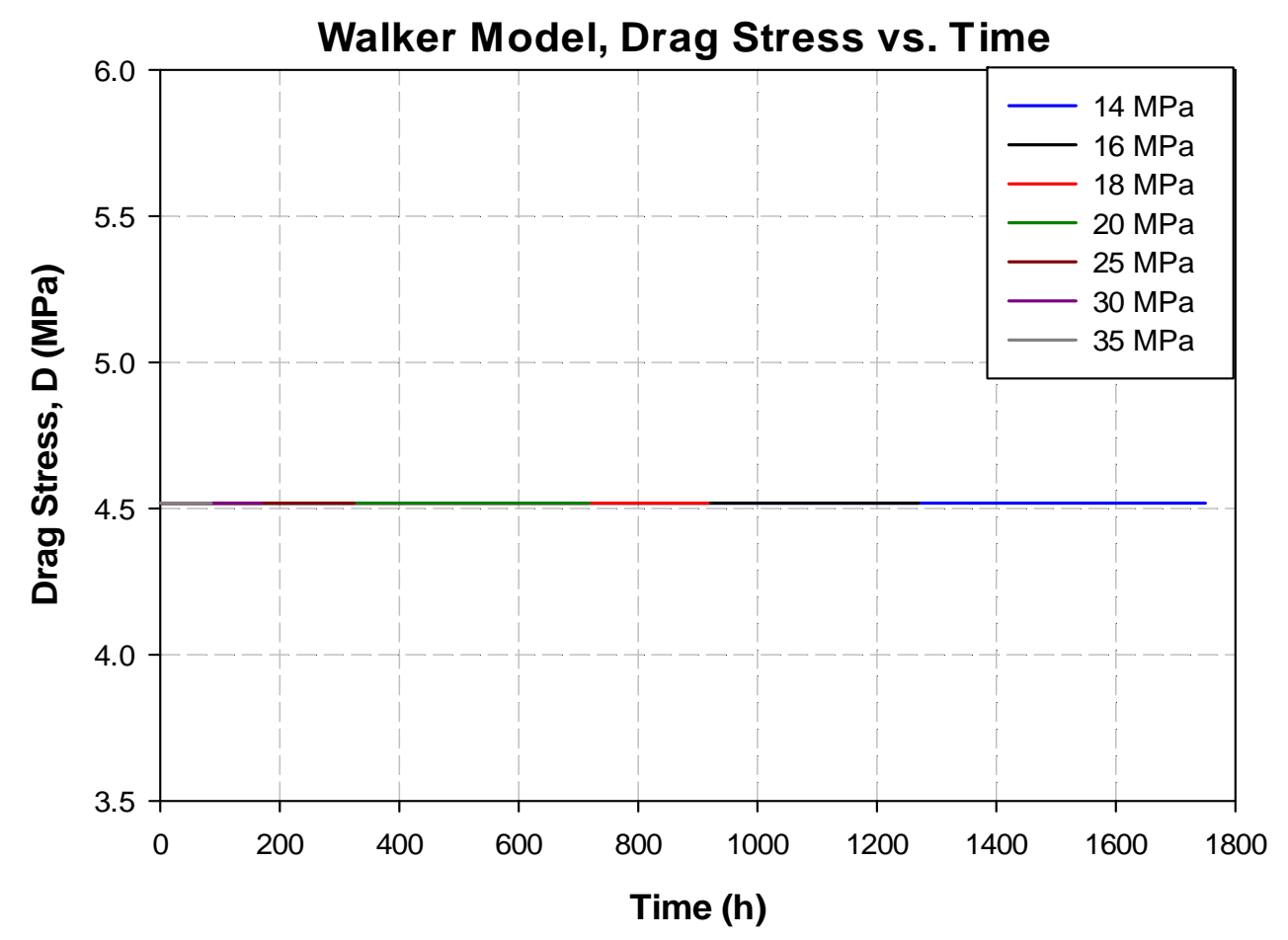

Figure 16: Walker model drag stress vs. time

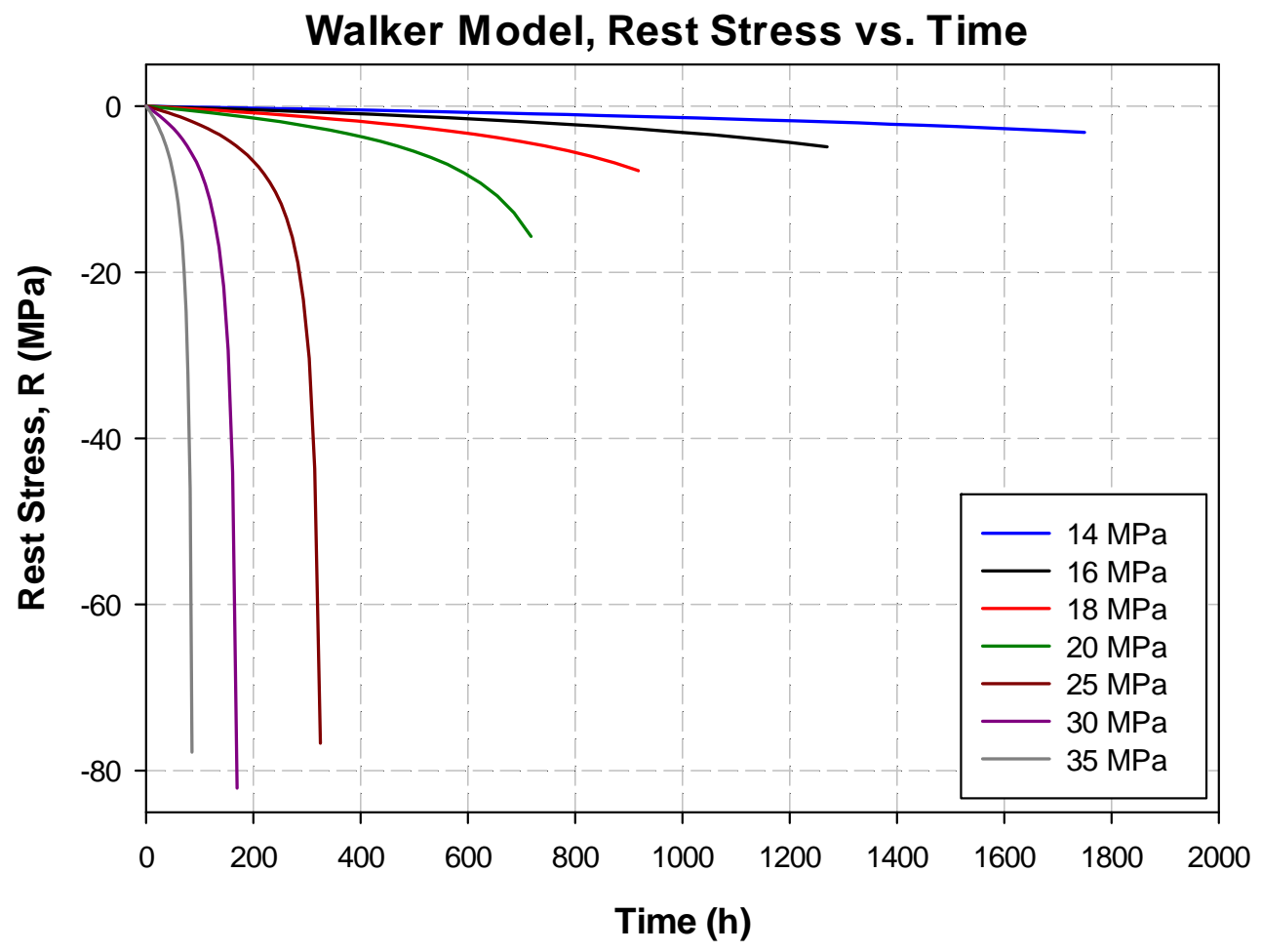

Figure 17: Walker model rest stress vs. time 
Miller and Walker models were exercised using the finite element calculation included in MACHO. ANSYS was not used to simulate the low cycle behavior of the models, because it didn't accepted the optimized material constant values optimized by MACHO because they seem to be unrealistic for the software. Besides there were some discrepancies between ANSYS and MACHO calculation of the updated stress, because MACHO calculates the updated stress assuming a uniaxial stress, whereas ANSYS calculates the updated stress assuming equivalent or Von Mises stress. Thus, the following numerical simulation results for the low cycle behavior of stainless steel 304 were performed using the MACHO internal finite element tool.

Figure 18 and Figure 19 show the low cycle fatigue numerical simulation results using the Miller model against the experimental data, at a temperature of $T=600^{\circ} \mathrm{C}$ and strain amplitudes of $\Delta \varepsilon=0.005$ and $\Delta \varepsilon=0.007$, respectively. From Figure 18 it can be observed how the numerical simulated curve exhibit a lower hardening prediction compared to experimental data in the hardening region. In the negative stress region, the numerical simulations display a much softer behavior than the experimental data. At this strain amplitude the Miller model under predicts low cycle fatigue behavior, since the predicted hardening and softening behavior are smaller than the actual behavior. In Figure 19 it can be observed how the hardening portion of the initial cycle is under predicted by the numerical simulated curve. Overall there is good correspondence between the simulated and the experimental curve; however at the maximum softening region (lower stress point) and at the middle hardening region (positive stress, negative strain area) the numerical simulated curve exhibits an under prediction of the behavior. 


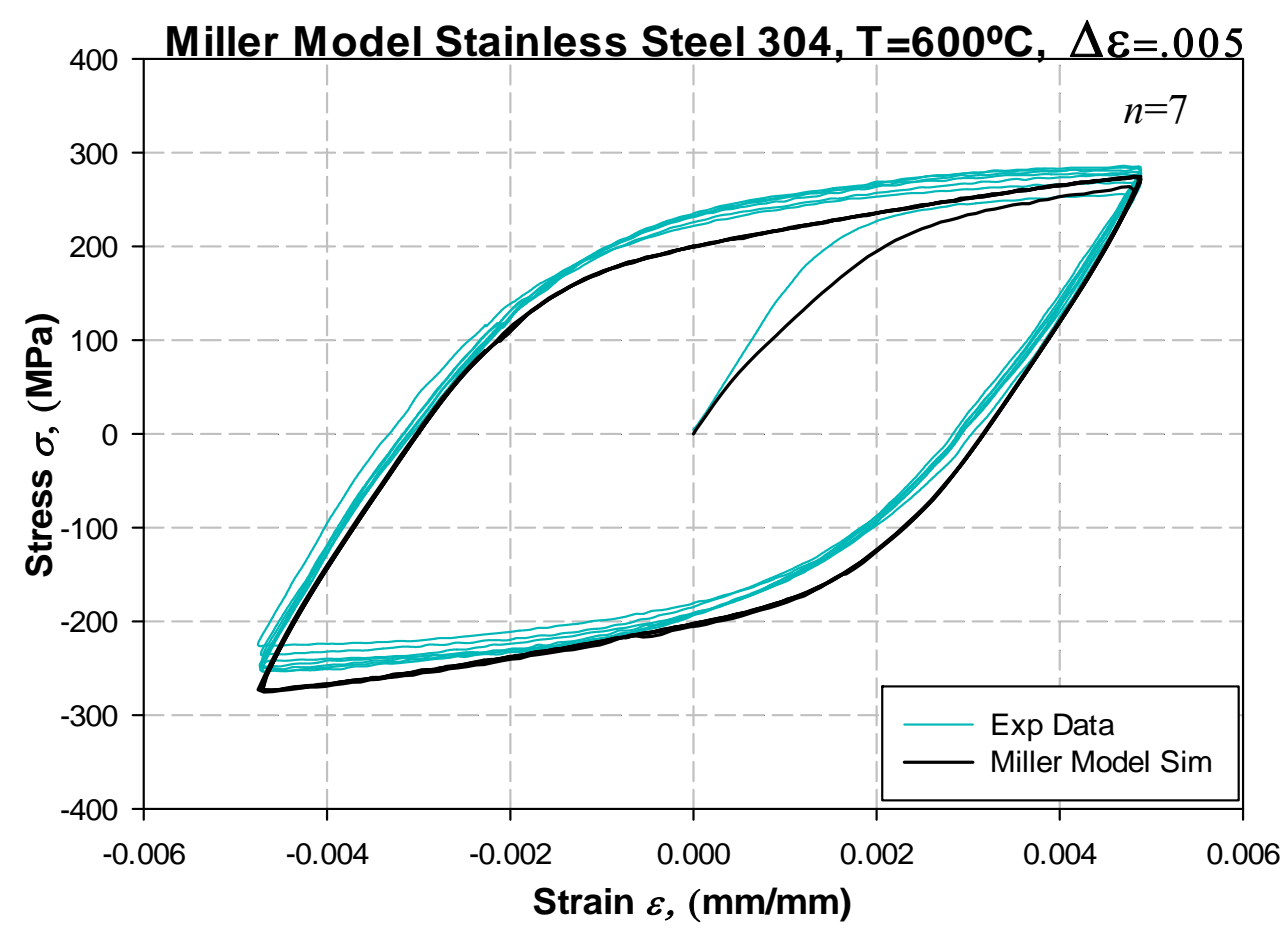

Figure 18: Miller model simulation vs. experimental data [68]

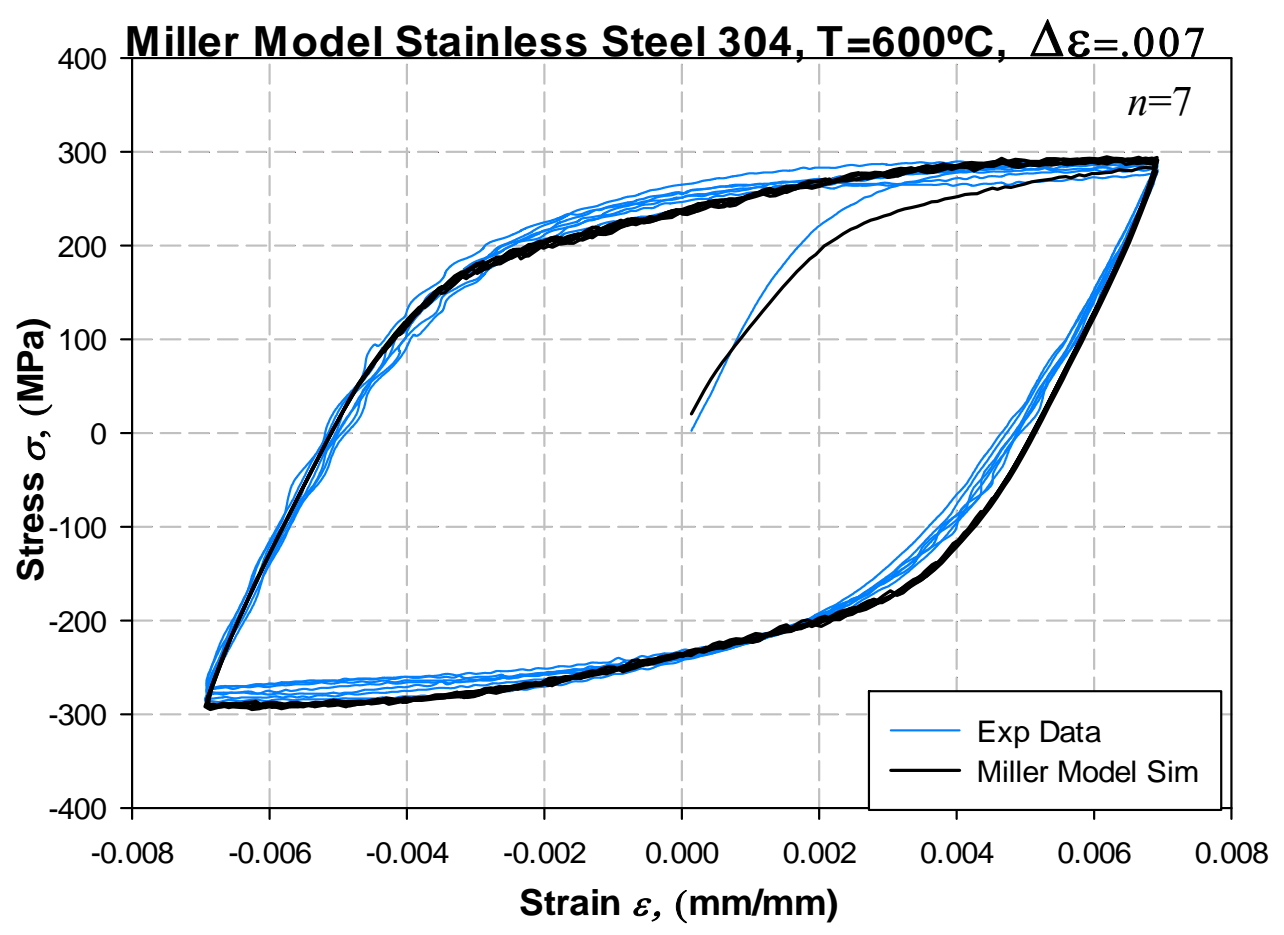

Figure 19: Miller model simulation vs. experimental data [68] 
Figure 20 and Figure 21 show the Walker model results prediction for low cycle fatigue against the experimental data, at a temperature of $T=600^{\circ} \mathrm{C}$ and strain amplitudes of $\Delta \varepsilon=0.005$ and $\Delta \varepsilon=0.007$, respectively. From Figure 20 at the maximum softening region (lower stress point) a good correspondence can be observed for the first cycle, whereas for the subsequent cycles there are some discrepancies since the behavior is under predicted. At the middle hardening region (positive stress, negative strain area) there is a small under prediction of the behavior; however, at the maximum hardening region (highest stress point) there is a good hardening prediction. From Figure 21 an overall close correspondence between simulated and experimental curves can be observed; however, there is a slight over prediction of the behavior at the maximum hardening region. In low cycle fatigue test the use of optimized material constants do not give any extra capabilities to the models, only a high accuracy level to describe the material behavior. No comparison figures of drag and rest stress for low cycle fatigue simulations are presented since the behavior of these state variables is agnostic to the physics due to the use of the optimized material constants. This issue is exemplified in creep simulation figures. 


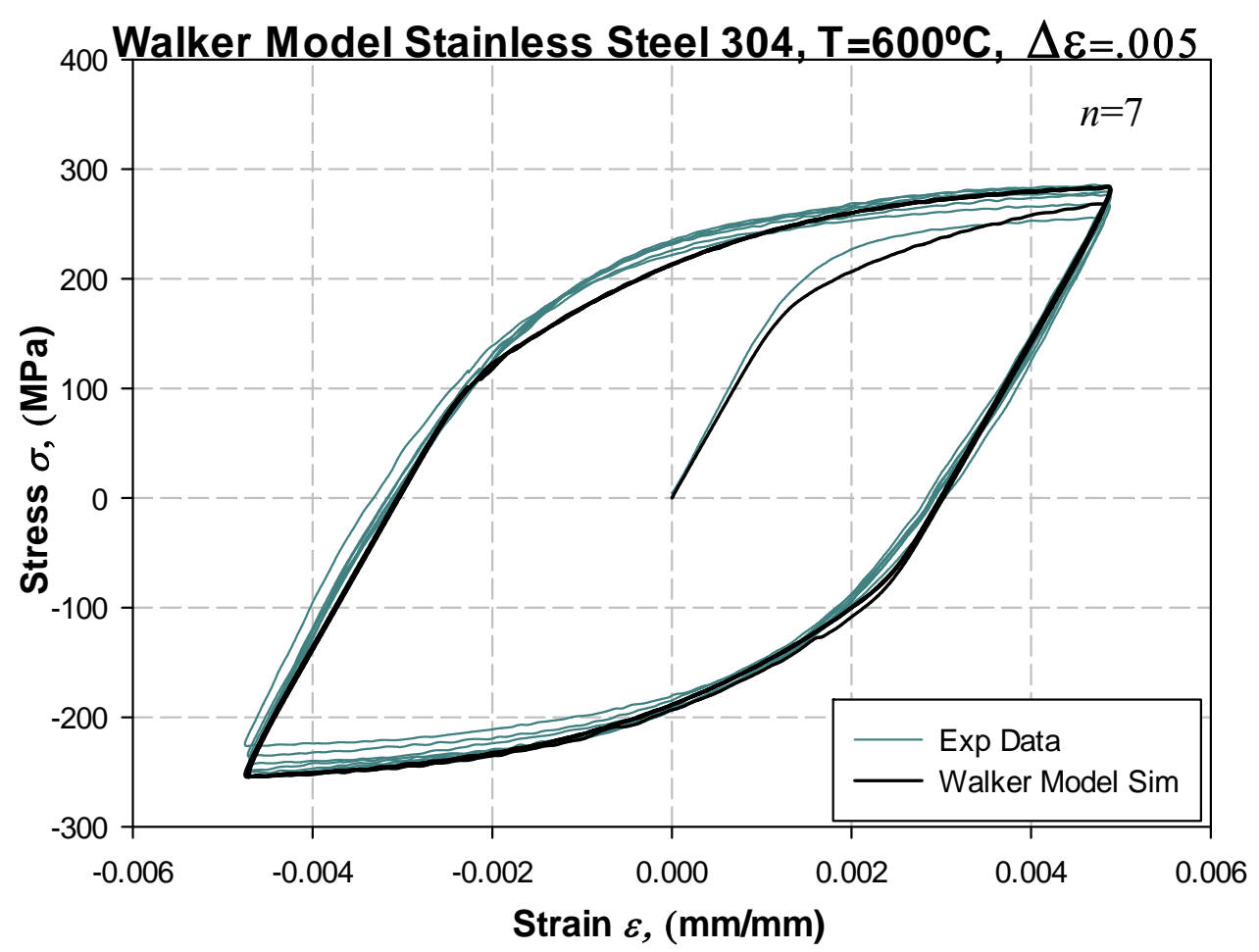

Figure 20: Walker model simulation vs. experimental data [68]

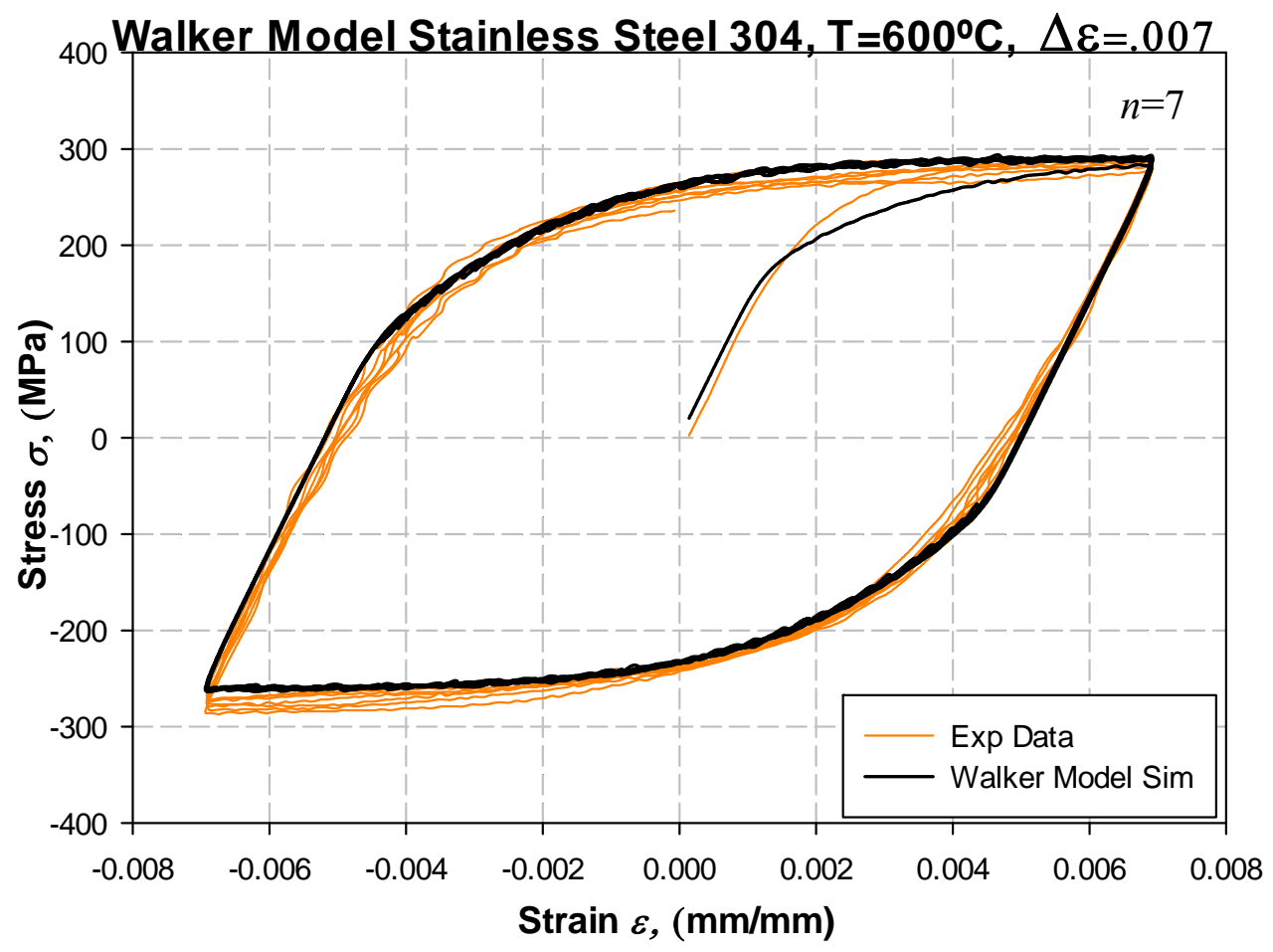

Figure 21: Walker model simulation vs. experimental data [68] 
In Figure 22 and Figure 23 show the stress amplitude (top and bottom stress levels) with respect to the number of cycles at strain amplitudes of 0.005 and 0.007 , respectively. In this figures the previously predicted stress amplitude (Figure 18-Figure 21) of each viscoplastic model is compared to the experimental data. Figure 22 shows a comparison of the viscoplastic behavior predictions and experimental data at an strain amplitude of 0.005 . From this figure it can be observed how Miller model is over predicting the hardening behavior during the first couple of cycles, meaning that the viscoplastic model is hardening at a higher rate compared to experimental data. In this figure it can be observed how Miller model is is exhibiting a good correspondence in the top stress levels or at the hardening points; however, at the lower stress points or softening points, the Miller model is under predicting the stress response at all points. On the other hand Walker model is better predicting the material hardening during the first couple of cycles; however, it is still being over predicted by Walker model. Considering the top stress levels or hardening points there is a better correspondence of Walker model predictions compared to those of Miller model. in the lower stress levels or softening points it can be observed how Walker model is closely following the softening path of the material described by the experimental data, meaning that Walker model is accurately predicting the softening behavior of the material.

Figure 23 shows the comparison of the viscoplastic model predictions with respect to experimental data at an strain amplitude of 0.007. From this figure it can be observed how Miller model is over predicting the hardening of the material during the first couple of cycles, as the cycle number increases and the material hardens the correspondence between Miller model predictions and experimental data increases. The softening behavior is being under predicted by Miller model at all stress points. On the other hand, Walker model is better predicting the 
softening behavior of the material; however, it is still under predicting the stress level. Considering the hardening of the material the Walker model is over predicting the hardening during the first couple of cycles, however, as the number of cycles increases the correspondence with respect to experimental data does it as well. Overall both viscoplastic models are not exhibiting a good correspondence to experimental data of the material hardening during the first couple of cycles, meaning that the predicted hardening of both models is not evolving as close to experimental data as expected that is why the highest accuracy or correspondence of both models is exhibited at a higher cycle number where the material hardening increases and tends to stabilize. 


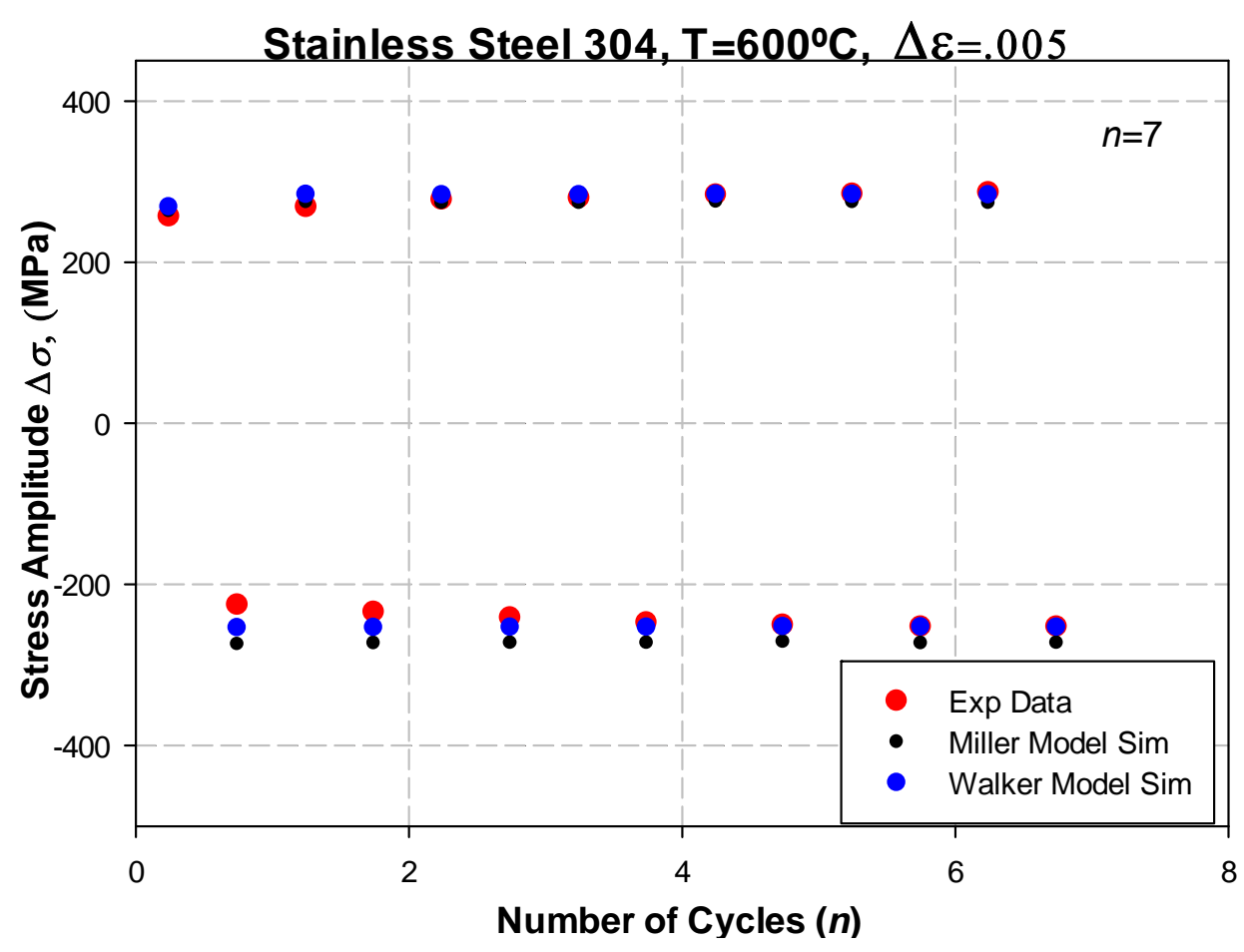

Figure 22: Comparison of stress amplitude vs. number of cycles at $\Delta \varepsilon=0.005$

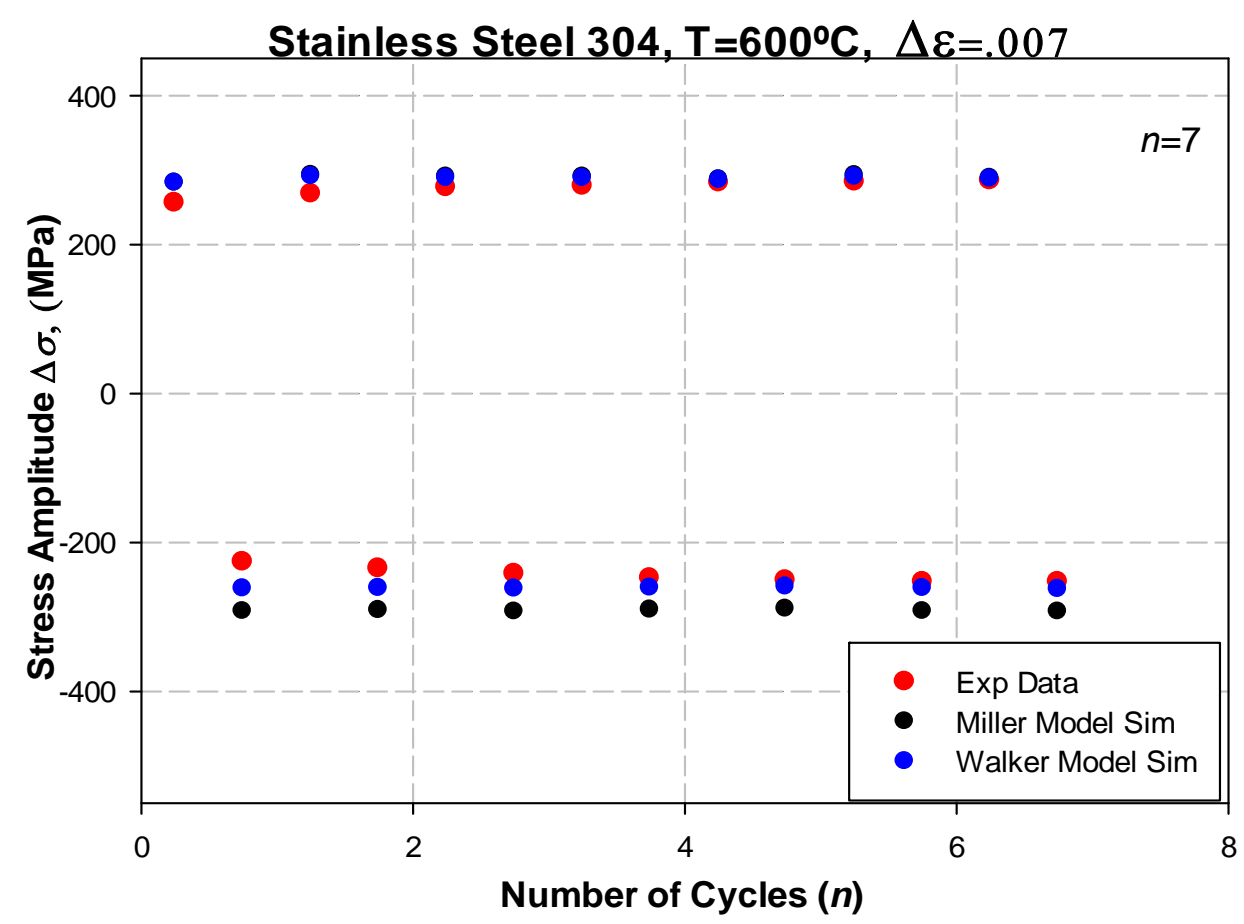

Figure 23: Comparison of stress amplitude vs. number of cycles at $\Delta \varepsilon=0.007$ 


\subsection{ANALYSIS}

In order to determine which constitutive model is the best to describe Hastelloy $\mathrm{X}$ and stainless steel 304 behaviors, it is required to consider the accuracy and the friendliness of each constitutive model. To facilitate the process of this decision, a quantitative and qualitative discussion is presented. To establish a numerical or quantitative evaluation of the behavior of each model with respect to the experimental data, the time step size of each model was set equal to the time step size of the experimental data. To do so, for Hastelloy X creep ten experimental data points were selected as a base time step size and by using linear interpolation, ten numerical simulation data points (strain and time) were obtained for each constitutive model at exactly the same time. With these new data points, the ten experimental data points and the twenty numerical simulation data points (ten from each model) were comparable since all the new strain points were calculated at the same time point. On the contrary, this procedure was not necessary for the low cycle fatigue data of stainless steel 304 since the time step size is the same between the numerical simulations and the experimental data. The mean percentage error (MPE) was calculated between the experimental data and the new numerical simulation data points for each constitutive model at each stress level for Hastelloy X. The results are presented in Table 7. Table 8 presents the MPE between the experimental data and numerical simulation results of stainless steel 304 subjected to low cycle fatigue. The equation used to calculate MPE was Eq.(26), where $n$ represents the total number of data points considered at each stress level and strain amplitude.

$$
M P E=\frac{100}{n} \sum\left|\frac{\#_{\text {SimData }}-\#_{\text {ExpData }}}{\#_{\text {ExpData }}}\right|
$$


The MPE represents the average percentage error by which the numerical simulated data differ from the experimental data. Therefore the smaller the MPE, the more accurate the constitutive model is. The coefficient of determination $\left(R^{2}\right)$ was also calculated for each stress level and strain amplitude. The coefficient of determination is a number that specifies how well the numerical simulated data fits the experimental data at each stress level. When $R^{2}=1$, it denotes that the numerical simulation data perfectly fits the experimental data; thus, the higher the coefficient of determination the more accurate the constitutive model is at a specific stress level or strain amplitude. The coefficient of determination $R^{2}$ was calculated using Eq.(27), which implied the use of the total sum of squares given by Eq.(28), which is proportional to the variance of the data and also the use of the sum of squares of residuals given by Eq.(29) also called the residual sum of squares. Where $i$ represent the number of data points consider at a specific stress level, $y_{i}$ and $f_{i}$ represent an experimental data point and a numerical simulated data point respectively, $\bar{y}$ is the mean value of the experimental data at a specific stress level. The coefficient of determination was calculated for each stress level and strain amplitude; the results are presented in Table 7 and Table 8, respectively.

$$
\begin{gathered}
R^{2}=1-\frac{S S_{\text {res }}}{S S_{\text {tot }}} \\
S S_{\text {tot }}=\sum_{i}\left(y_{i}-\bar{y}\right)^{2} \\
S S_{\text {res }}=\sum_{i}\left(y_{i}-f_{i}\right)^{2}
\end{gathered}
$$

According to the results presented in Figure 12 at high stress levels where $\sigma \geq 18 \mathrm{MPa}$ Walker model exhibited the smaller MPE and the higher $R^{2}$ values, meaning that Walker model produces better predictions than Miller model at these stress levels, where primary, secondary 
and tertiary creep are predicted. However, at $30 \mathrm{MPa}$ MPE difference between Miller and Walker model is not significant. In accordance with Figure 12 and Figure 15 where it was shown how the numerical simulation data graphically fits the experimental data, the results on Table 7 show how the accuracy and precision of each constitutive model decreases as the stress level decreases. At $\sigma \leq 16 \mathrm{MPa}$, the Miller model exhibited a higher accuracy than the Walker model. However, at these stress levels both models are only predicting primary and secondary creep. So at high stress levels $\sigma \geq 18 \mathrm{MPa}$, Walker model exhibited a higher accuracy than Miller model by $2.23 \%$ MPE. On the other hand at low stresses $\sigma \leq 16 \mathrm{MPa}$, Miller model exhibited a higher accuracy than Walker model by $1.903 \% \mathrm{MPE}$.

Table 7: Calculated mean percentage error (MPE) and coefficient of determination $\left(R^{2}\right)$ for Miller and Walker numerical simulations for Hastelloy X subjected to creep

\begin{tabular}{ccccc}
\hline & \multicolumn{2}{c}{ Miller Model } & \multicolumn{2}{c}{ Walker Model } \\
Stress Level & MPE & $R^{2}$ & MPE & $R^{2}$ \\
\hline $35 \mathrm{MPa}$ & 15.15029 & 0.983132 & 10.52089 & 0.983448 \\
\hline $30 \mathrm{MPa}$ & 15.9161 & 0.622079 & 15.62258 & 0.798828 \\
\hline $25 \mathrm{MPa}$ & 14.6515 & 0.700181 & 10.91014 & 0.926826 \\
\hline $20 \mathrm{MPa}$ & 16.17862 & 0.581108 & 15.09627 & 0.698367 \\
\hline $18 \mathrm{MPa}$ & 35.69324 & 0.070061 & 33.29827 & 0.099627 \\
\hline $16 \mathrm{MPa}$ & 47.52395 & -0.17722 & 48.50205 & -0.26891 \\
\hline $14 \mathrm{MPa}$ & 54.40565 & -0.35598 & 56.69404 & -0.49785 \\
\hline
\end{tabular}

According to the results presented in Table 8 at an strain amplitude of $\Delta \varepsilon=0.005$, Walker model exhibit a smaller MPE compared to Miller model, considering the MPE Walker model is 43.60\% more accurate than Miller model. Considering the coefficient of determination Walker model produces the highest value, meaning that there is a higher correspondence between its prediction and the experimental data. At an strain amplitude of $\Delta \varepsilon=0.007$ Miller model 
presented a smaller coefficient of determination, meaning that there is a smaller correspondence between its prediction and the experimental data. Considering the MPE Walker model exhibit a smaller value; therefore, at this strain amplitude Walker model is $0.1591 \%$ MPE more accurate than Miller model.

Table 8: Calculated mean percentage error (MPE) and coefficient of determination $\left(R^{2}\right)$ for Miller and Walker numerical simulations for stainless steel 304 subjected to low cycle fatigue

\begin{tabular}{ccccc}
\hline Strain & \multicolumn{2}{c}{ Miller Model } & \multicolumn{2}{c}{ Walker Model } \\
Amplitude & MPE & $R^{2}$ & MPE & $R^{2}$ \\
\hline 0.005 & 47.547 & 0.986091 & 26.814 & 0.996177 \\
\hline 0.007 & 23.139 & 0.994617 & 22.688 & 0.996202 \\
\hline
\end{tabular}

Walker model involves the use of nine material constants to predict the same phenomena as Miller model, whereas Miller model is using only seven material constants; therefore, this is a point in favor for Miller model. The procedure to calculate Walker constants is not well documented, while Miller model procedure to calculate material constants is explained and documented by Miller [51, 53]; thus a second point in favor for Miller model. In terms of equations Walker model is a more complex constitutive model since it consists of five constitutive equations. On the other hand, the Miller model is simpler since it only involves the use of three constitutive equations; therefore, a third point in favor of Miller model. However, its sine hyperbolic function makes it difficult to manipulate. The Miller model is demonstrated to be physically more competent than the Walker model in describing drag and rest stress behavior during numerical simulations. However, in terms of accuracy as demonstrated in this paper the Walker model produces a higher accuracy level. 


\section{CHAPTER 6: DEVELOPMENT AND EXERCISE OF A NOVEL HYBRID UNIFIED VISCOPLASTIC MODEL}

The development of the novel hybrid unified viscoplastic model has been performed in three stages. The first stage is hybrid model V0, consists on the development of a basic unified viscoplastic model formed by the inelastic strain rate, rest stress rate and drag stress equations. After exercise the hybrid model V0, the deficiencies are analyzed and a hybrid model V1 is proposed which is based on hybrid model V0 with further improvements. Hybrid model V1 contains an improved rest stress rate equation and a damage law is integrated in the constitutive equations of the model. Finally a hybrid model V2 is presented, which is an improvement of hybrid model V1. In the hybrid model V2 the drag stress equation is modified to improve the fatigue prediction capabilities of the model. In all three development stages the main objective has been to keep the model constitutive equations in a simple format and with a low number of material constants. The accomplishment of this objective will lead to a easy to use model with low computation time when used to simulate.

\subsection{HYBRID MODEL VO}

Based on the basic theory of viscoplastic models, the best aspects of the analyzed Miller and Walker viscoplastic models, the unified viscoplastic model hybrid model V0 is proposed. The hybrid model V0 consists of the inelastic strain rate, rest stress rate and drags stress equations, which involves the use of five material constants that were determined using MACHO and experimental data, and two state variables (inelastic strain rate and rest stress). Hybrid model V0 possesses capabilities to model the inelastic behavior of materials during low cycle fatigue, primary and secondary creep at isothermal conditions. 


\subsubsection{MODEL DEVELOPMENT}

The inelastic strain rate equation of the hybrid model V0 is presented in Eq. (30) . This equation incorporates the hyperbolic sine function of the Miller model and the power function of the Walker model. The hyperbolic sine function was selected because its mathematical nature will facilitate the prediction of creep. Just as in the basic skeleton of viscoplastic models, this hybrid model is function of the applied stress $\sigma$, the state variables rest $(R)$ and drag $(D)$ stress. The signum (sgn) function accommodates reversed stress flow allowing the model to predict fatigue. Material constant $B$ adjusts the overall precision magnitude of the inelastic strain rate, while constant $n$ controls the stress intensity in the viscoplastic model.

$$
\dot{\varepsilon}=B \sinh \left(\frac{|\sigma-R|}{D}\right)^{n} \operatorname{sgn}(\sigma-R)
$$

The rest stress rate equation follows the basic hardening-softening format for the hardening equations, it is presented in Eq. (31). Just as in Miller and Walker constitutive models the hardening term consist only on the inelastic strain rate $\dot{\varepsilon}$ and the hardening material constant $H_{l}$, the hardening constant proportionally controls the hardening magnitude of the rest stress. Following Walker simple softening term, a dynamic or recovery term is included. This softening term is function of the inelastic strain rate $\dot{\varepsilon}$, the accumulated rest stress $(R)$ state variable and the softening material constant $S_{l}$. The magnitude of the softening constants controls the rest stress softening behavior.

$$
\dot{R}=H_{1} \dot{\varepsilon}-R S_{1} \dot{\varepsilon}
$$

By following Walker assumption [56] no hardening or softening term is included for the drag stress equation Eq.(32) . The drag stress equation is a non-evolutionary equation, meaning that 
the drag stress equation consists only on a constant value represented by material constant $\mathrm{H}_{2}$. Therefore, the magnitude of the drag stress is dictated by the value of material constant $\mathrm{H}_{2}$.

$$
D=H_{2}
$$

\subsubsection{EXERCISE OF HYBRID MODEL VO}

Table 9 show the initial guess material constants values used by MACHO during the optimization process for Hastelloy X creep, and the resultant optimized material constants values which were used for the simulation and exercise of the proposed hybrid model V0. The optimization process consisted of the optimization of seven creep experimental data sets (35 $\mathrm{MPa}, 30 \mathrm{MPa}, 20 \mathrm{MPa}, 18 \mathrm{MPa}$, and $16 \mathrm{MPa}$ ) for 50,000 iterations, with a temperature reduction factor of 0.25 and $125^{\circ} \mathrm{C}$ as the initial temperature.

Table 9: Hastelloy X creep material constants for hybrid model V0

\begin{tabular}{cccc}
\hline $\begin{array}{c}\text { Material } \\
\text { Constant }\end{array}$ & $\begin{array}{c}\text { Initial Guess } \\
\text { Value }\end{array}$ & $\begin{array}{c}\text { Optimized } \\
\text { Value }\end{array}$ & Units \\
\hline $\boldsymbol{B}$ & $0.71611 \mathrm{e}-7$ & $0.52565 \mathrm{e}-7$ & $\mathrm{Sec}^{-1}$ \\
\hline $\boldsymbol{n}$ & 0.52218 & 0.50519 & - \\
\hline $\boldsymbol{H}_{\boldsymbol{I}}$ & 1.8844 & 1.9139 & - \\
\hline $\boldsymbol{H}_{\boldsymbol{2}}$ & 0.32765 & 0.26577 & $\mathrm{MPa}$ \\
\hline $\boldsymbol{S}_{\boldsymbol{I}}$ & 172.71 & 173.16 & - \\
\hline Obj Funct. & $2,122.30$ & $2,097.172$ & - \\
\hline
\end{tabular}

Two different alloys are considered in the present work to test the flexibility and capabilities of the proposed constitutive equations and $\mathrm{MACHO}$ algorithms to model the inelastic behavior of multiple materials. Stainless steel 304 was used to test the modeling capabilities at low cycle fatigue conditions. The optimization parameters used for low cycle fatigue optimization process were, 60,000 iterations an initial temperature of $125^{\circ} \mathrm{C}$ and a temperature reduction factor of 0.25 . 
Table 10 show the initial guess material constants values used by MACHO during the optimization process for 304 stainless steel low cycle fatigue, and the resultant optimized material constants values which were used for the simulation and exercise of the proposed hybrid model.

Table 10: 304 Stainless steel low cycle fatigue material constants for hybrid model V0

\begin{tabular}{cccc}
\hline $\begin{array}{c}\text { Material } \\
\text { Constant }\end{array}$ & $\begin{array}{c}\text { Initial Guess } \\
\text { Value }\end{array}$ & $\begin{array}{c}\text { Optimized } \\
\text { Value }\end{array}$ & Units \\
\hline $\boldsymbol{B}$ & $0.33238 \mathrm{e}-3$ & $0.15355 \mathrm{e}-2$ & $\mathrm{Sec}^{-1}$ \\
\hline $\boldsymbol{n}$ & 1.6723 & 1.8917 & - \\
\hline $\boldsymbol{H}_{\boldsymbol{1}}$ & 7226.1 & $6,622.4$ & - \\
\hline $\boldsymbol{H}_{\boldsymbol{2}}$ & 175.22 & 335.56 & $\mathrm{MPa}$ \\
\hline $\boldsymbol{S}_{\boldsymbol{I}}$ & 3.4251 & $0.10757 \mathrm{e}-9$ & - \\
\hline Obj Funct. & $3,735.03$ & $3,608.72$ & - \\
\hline
\end{tabular}

The objective function values of the fatigue and creep simulations cannot be compared between them, because of two main reasons. First, the time duration of the fatigue test is much smaller (140 190 seconds) compared to creep test (77 1,200 hours); therefore, the importance or weight of each experimental type is different for MACHO. The optimization software would focus more (assign more weight or importance) on the optimization of the creep experimental data since it has a larger duration of time, whereas the fatigue experimental data would have least importance because of the shorter duration. Second, two different material are being optimized and the material constants are characteristic of each alloy. The proposed hybrid model V0 was exercised using the FEMCREEP feature of MACHO to perform numerical finite element (FE) simulations of creep and low cycle fatigue at multiple stress and strain amplitude levels, while at elevated temperature levels. The proposed hybrid model V0 was exercised using the FEMCREEP feature of MACHO to perform numerical finite element (FE) simulations of creep 
and low cycle fatigue at multiple stress and strain amplitude levels, while at elevated temperature levels. The numerical simulation results are compared to experimental data in Figure 27- Figure 26. Figure 27 shows the creep results of Hastelloy $\mathrm{X}$ at $950^{\circ} \mathrm{C}$ at different stress levels ( $35 \mathrm{Mpa}$. $30 \mathrm{MPa}, 20 \mathrm{MPa}, 18 \mathrm{MPa}, 16 \mathrm{MPa}$ ). In the other hand, Figure 25 and Figure 26 shows the low cycle fatigue results of 304 Stainless Steel at $600^{\circ} \mathrm{C}$, at strain amplitudes $(\Delta \varepsilon)$ of 0.005 and 0.007 .

From Figure 24 it can be observed how hybrid model V0 is capable of predicting primary and secondary creep. The resultant straight line in the figures represents the secondary creep behavior, which shows a good correspondence between the experimental data and the simulated creep. However this figure demonstrates that the hybrid model V0 does not have capabilities to describe tertiary creep; therefore further improvement is required. From Figure 25 it can be observed how the hybrid model V0 exhibits a good correspondence with experimental data predicting the top or maximum stress point, whereas the lower stress point is under predicted by the hybrid model. There is a good correspondence between the experimental data and the simulated data on the hardening behavior of the hysteresis loop. On the other hand, the softening behavior is under predicted by the hybrid model. Figure 26 shows a better overall correspondence between the experimental and simulated data. The hardening behavior is not accurately predicted; however, the softening behavior is better predicted at this strain amplitude but there are still some discrepancies. The maximum stress point is over predicted, whereas the minimum stress is under predicted by the hybrid model V0. These observations lead to the conclusion that a improvement of the softening term is required. 


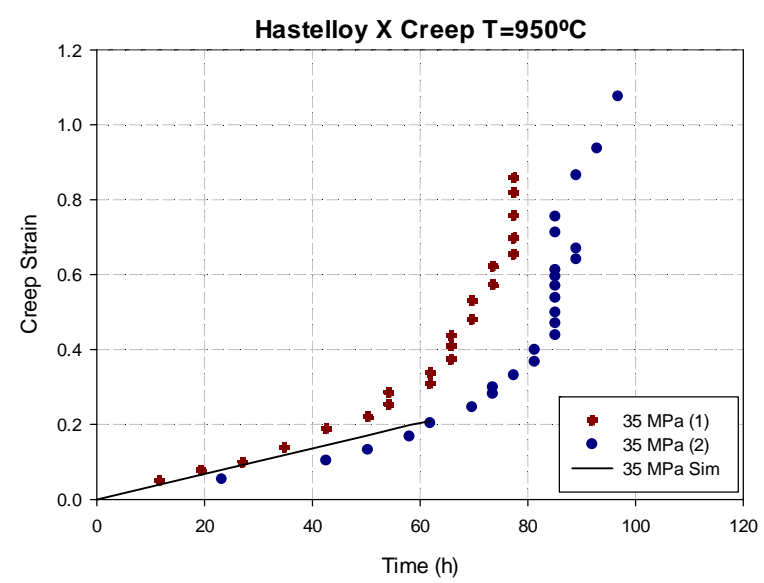

(a)

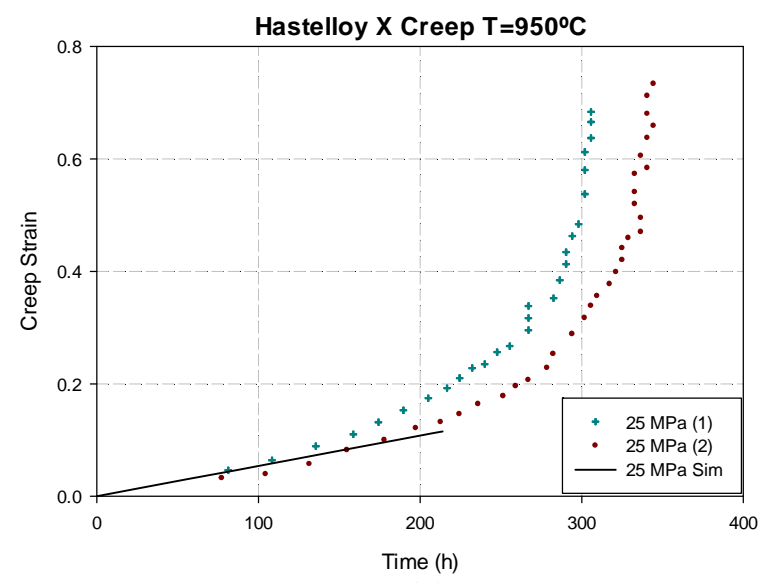

(c)

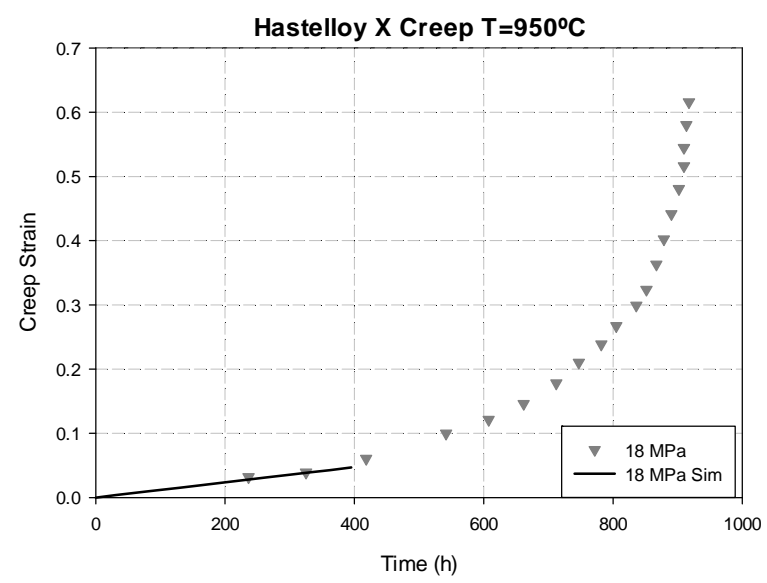

(e)

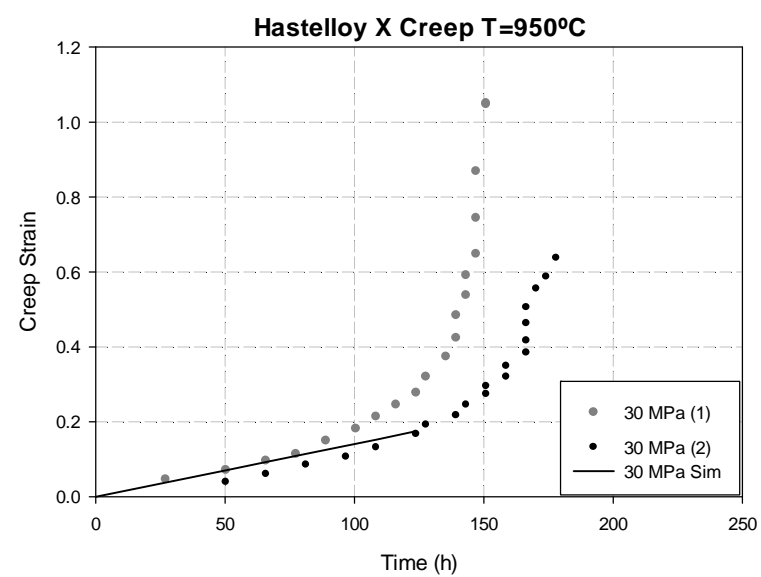

(b)

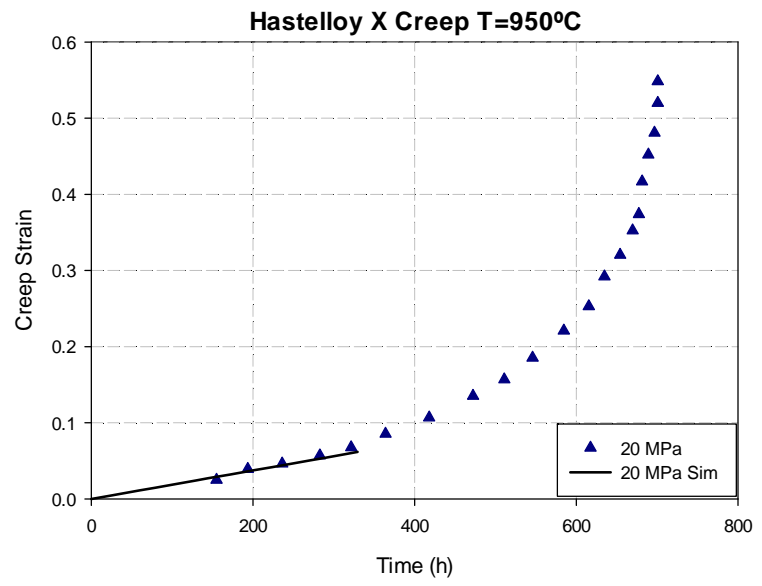

(d)

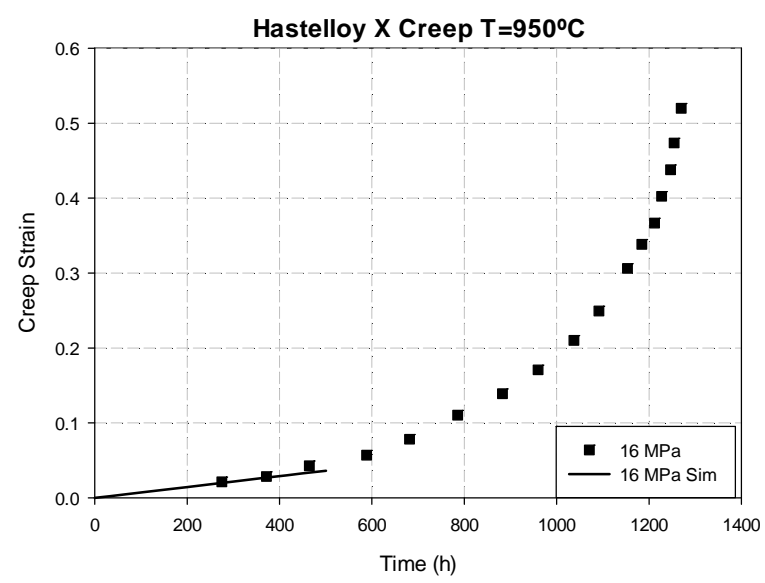

(f)

Figure 24: Hastelloy X creep vs hybrid model V0 simulation at multiple stress levels 


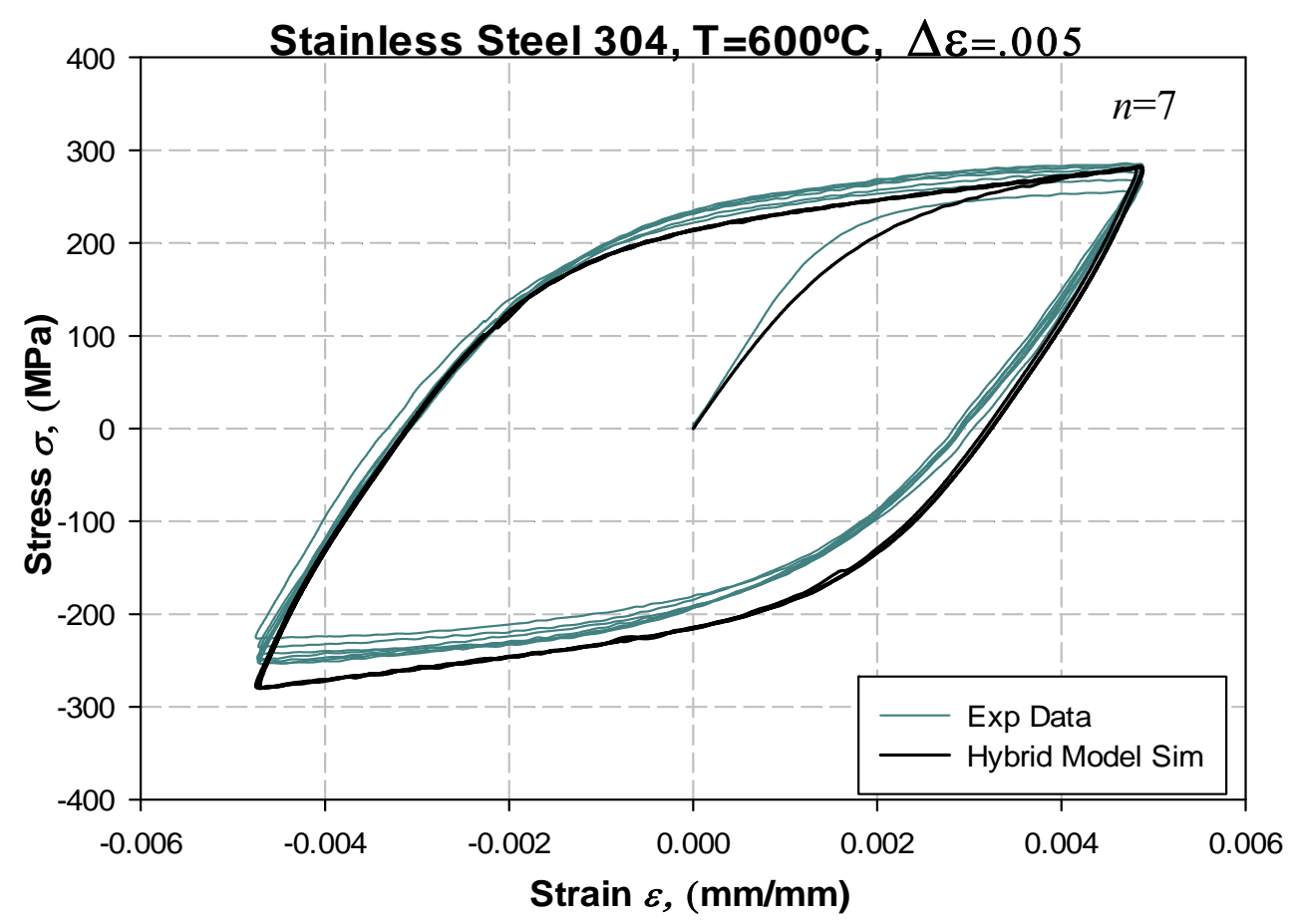

Figure 25: 304 Stainless steel low cycle fatigue vs. hybrid model V0 simulation at $\Delta \varepsilon=0.005$

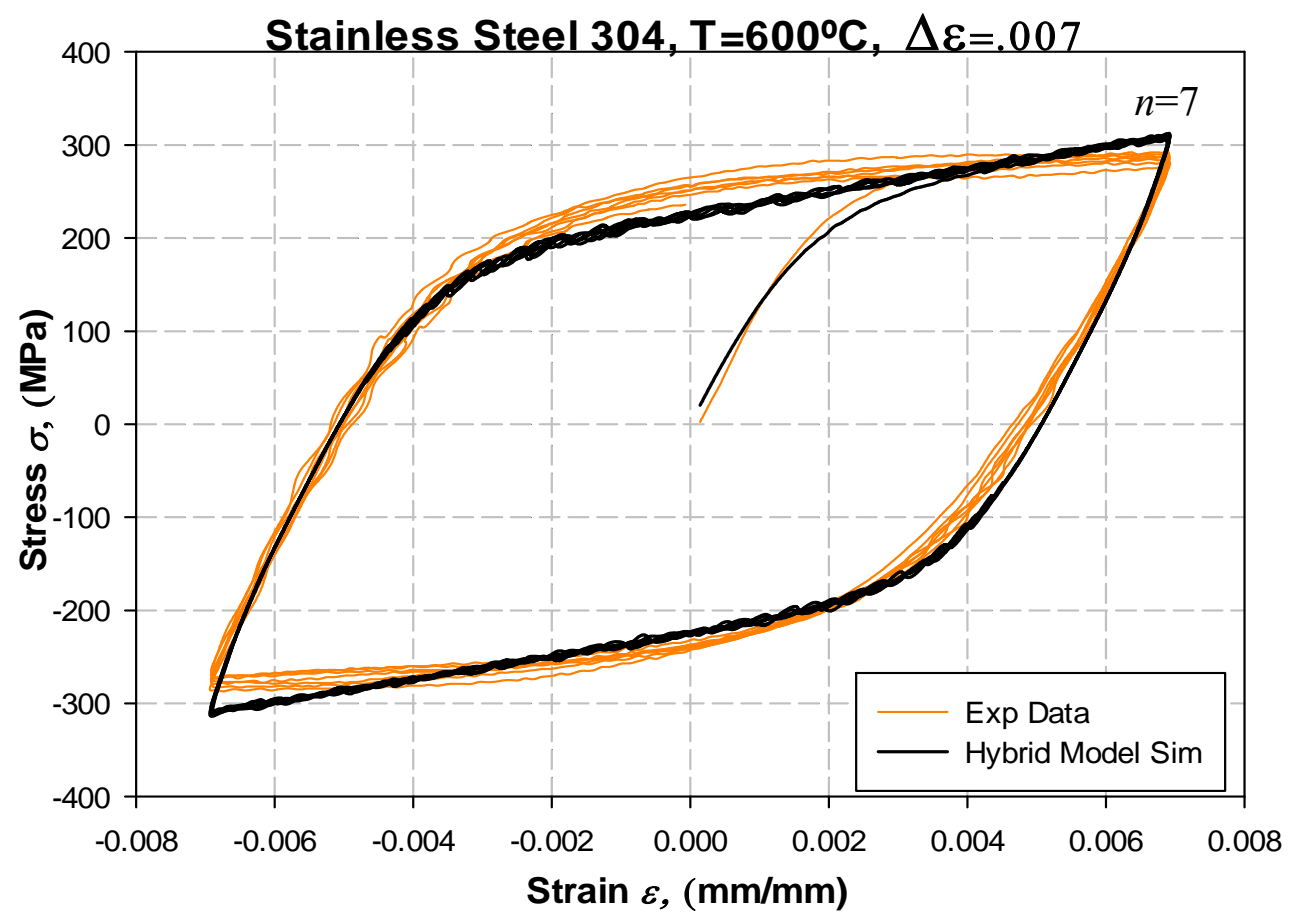

Figure 26: 304 Stainless steel low cycle fatigue vs. hybrid model V0 simulation at $\Delta \varepsilon=0.007$ 
To determine the hybrid model V0 accuracy describing Hastelloy X and stainless steel 304 behaviors, a quantitative discussion is presented. To establish a numerical or quantitative evaluation on the behavior the hybrid model with respect to the experimental data, the mean percentage error (MPE) and the coefficient of determination $\left(R^{2}\right)$ were calculated. The mean percentage error (MPE) was calculated between the experimental data and the numerical simulation data points for each data point at each stress level for Hastelloy X, only the primary and secondary data was consider for calculation of the MPE and $R^{2}$. The results are presented in Table 11. Table 12 presents the MPE between the experimental data and numerical simulation results of stainless steel 304 subjected to low cycle fatigue.

From Table 11 it can be observed that the higher accuracy of the hybrid model V0 predicting creep is at $30 \mathrm{MPa}$, where the lowest MPE and the highest $R^{2}$ are present. The correspondance between experimental data and simulated data is at $20 \mathrm{MPa}$, where the highest MPE and the lowest $R^{2}$ are present.

Table 11: Mean percentage error (MPE) and coefficient of determination $\left(R^{2}\right)$ of Hastelloy X creep simulations hybrid model V0

\begin{tabular}{ccc}
\hline Stress Level & MPE (\%) & $\boldsymbol{R}^{\mathbf{2}}$ \\
\hline $35 \mathrm{MPa}$ & 13.57184 & 0.903691 \\
\hline $30 \mathrm{MPa}$ & 4.287146 & 0.947752 \\
\hline $20 \mathrm{MPa}$ & 27.9802 & 0.292467 \\
\hline $18 \mathrm{MPa}$ & 8.113803 & 0.93126 \\
\hline $16 \mathrm{MPa}$ & 8.266703 & 0.910554 \\
\hline
\end{tabular}


According to Table 12, hybrid model V0 exhibits its higher accuracy at 0.007 strain amplitude where the lowest MPE and the higher coefficient of determination are present. This numerical conclusion matches the visual inspection discussed above. The strain amplitude 0.005 exhibits a higher MPE error and lower coefficient of determination, since lees correspondence between experimental data and simulated data is resulted. As discussed above, the lower correspondence is at this strain amplitude is due to the miss-prediction of the softening behavior of the material.

Table 12: Mean percentage error (MPE) and coefficient of determination $\left(R^{2}\right)$ of 304 stainless steel data low cycle fatigue simulations hybrid model V0

\begin{tabular}{ccc}
\hline Strain Amplitude & MPE & $\boldsymbol{R}^{\mathbf{2}}$ \\
\hline 0.005 & 41.253 & 0.986269 \\
\hline 0.007 & 19.750 & 0.991324 \\
\hline
\end{tabular}




\subsection{HYBRID MODEL V1}

Based on the developed hybrid model V0 presented in the previous section, a damage law was included to extend the model's capabilities to account for tertiary creep. Besides major modifications were performed to the softening term of the rest stress rate equation, to increase the model accuracy predicting the material softening during low cycle fatigue. The proposed hybrid model V1 consists of eight material constants and 4 state variables (rest stress, accumulated inelastic strain rate, inelastic strain and damage) to accommodate the internal material's state. The proposed hybrid model is functional for isothermal tests, since no temperature term is present. The hybrid model V1 is capable of modeling monotonic tension, low cycle fatigue, primary, secondary and tertiary creep at different stress and strain amplitude levels.

\subsubsection{MODEL DEVELOPMENT}

Haque and Stewart [61] sine hyperbolic damage model described in section 2.4.2, is used in the proposed hybrid model to add tertiary creep prediction capabilities. The damage evolution equation used is represented by Eq.(33).

$$
\dot{\omega}=\frac{M[1-\exp (-\phi]}{\phi} \sinh \left(\frac{\sigma}{\sigma_{t}}\right)^{3} \exp (\phi \omega)
$$

Following basic hardening equation format proposed by Frederick-Armstron [71], an accumulated inelastic strain rate and the previous step rest stress value are included in the softening term to account for the rest stress history throughout the test. Several mathematical functions were tested for the softening term in rest stress rate equation. The different mathematical functions were tested using $\mathrm{MACHO}$, and the best function was determined by the lowest objective function value that resulted from the exercise of the equation. The test consisted 
on simulating fatigue for 20,000 iterations where the initial guess values were the same for all the tested cases. Table 13 shows the different mathematical functions tested and the resultant objective function value.

Table 13: Rest stress rate equations tested for improvement

\begin{tabular}{cc}
\hline Rest Stress rate Equation & Objective Function Value \\
\hline$\dot{R}=H_{1} \dot{\varepsilon}-S_{1}|R| \dot{\varepsilon}^{p}$ & $3,788.97$ \\
\hline$\dot{R}=H_{1} \dot{\varepsilon}-S_{1} R \dot{\varepsilon}^{p}$ & $268,190.22$ \\
\hline$\dot{R}=H_{1} \dot{\varepsilon}-S_{1} \sinh (|R|) \dot{\varepsilon}^{p}$ & $8,675.521$ \\
\hline$\dot{R}=H_{1} \dot{\varepsilon}-S_{1} \exp (R) \dot{\varepsilon}^{p}$ & $8,682.93$ \\
\hline$\dot{R}=H_{1} \dot{\varepsilon}-S_{1} R^{2} \dot{\varepsilon}^{p}$ & $5,730.06$ \\
\hline$\dot{R}=H_{1} \dot{\varepsilon}-S_{1}|R|^{2} \dot{\varepsilon}^{p}$ & $7,243.73$ \\
\hline
\end{tabular}

From the mathematical functions tested, the best overall equation with the lowest objective function value resulted to be the first equation where the absolute value of the rest stress is used. The rest stress rate equation Eq. (34) of the hybrid model V1 consists of the hardening term which is function of the inelastic strain rate, and the dynamic recovery term which is function of an accumulated inelastic strain rate described by Eq.(35). The dynamic recovery or softening term is dependent on softening constant $S_{1}$, the major modification in the softening term are addition of the absolute value of the rest stress (state variable) and the accumulated inelastic strain rate (state variable), just as in the Walker model.

$$
\begin{gathered}
\dot{R}=H_{1} \dot{\varepsilon}-S_{1} \dot{\varepsilon}_{p}|R| \\
\dot{\varepsilon}_{p}=|\dot{\varepsilon}|
\end{gathered}
$$

The drag stress is represented by Eq.(36), which keeps the same structure as in hybrid model V0 where a non-evolutionary drag stress function is used.

$$
D=H_{2}
$$


The new inelastic strain rate equation is presented in Eq. (37). This equation includes the previously presented flow law equation with the addition of the exponential (exp) function, which incorporate the damage effects in the inelastic strain rate. The tem $\lambda$ is not counted as a material constants since it can be determined directly from experimental data by using Eq. (38).

$$
\begin{gathered}
\dot{\varepsilon}=B \sinh \left(\frac{|\sigma-R|}{D}\right)^{n} \operatorname{sgn}(\sigma-R) \exp \left(\lambda \omega^{\frac{3}{2}}\right) \\
\lambda=\ln \left(\frac{\dot{\varepsilon}_{\text {max }}}{\dot{\varepsilon}_{\text {min }}}\right)
\end{gathered}
$$

\subsubsection{EXERCISE OF HYBRID MODEL V1}

Table 14 show the initial guess material constants values used by MACHO during the optimization process for Hastelloy X creep, and the resultant optimized material constants values which were used for the simulation and exercise of the proposed hybrid model V1.

Table 14: Hastelloy X Creep Material Constants for hybrid model V1

\begin{tabular}{cccc}
\hline $\begin{array}{c}\text { Material } \\
\text { constant }\end{array}$ & $\begin{array}{c}\text { Initial guess } \\
\text { value }\end{array}$ & $\begin{array}{c}\text { Optimized } \\
\text { value }\end{array}$ & Units \\
\hline $\boldsymbol{B}$ & $0.2702 \mathrm{e}-3$ & $0.23945 \mathrm{e}-3$ & Sec $^{-1}$ \\
\hline $\boldsymbol{n}$ & 0.95161 & $0.23586 \mathrm{e}-2$ & - \\
\hline $\boldsymbol{H}_{\boldsymbol{I}}$ & $0.72027 \mathrm{e} 20$ & 737.02 & - \\
\hline $\boldsymbol{H}_{\boldsymbol{2}}$ & $0.34271 \mathrm{e} 20$ & $0.1184 \mathrm{e} 12$ & $\mathrm{MPa}$ \\
\hline $\boldsymbol{S}_{\boldsymbol{I}}$ & $0.23249 \mathrm{e} 9$ & $0.49514 \mathrm{e} 19$ & - \\
\hline $\boldsymbol{M}$ & $0.31266 \mathrm{e} 20$ & $0.31707 \mathrm{e} 20$ & - \\
\hline$\phi$ & $0.60955 \mathrm{e} 20$ & $0.53055 \mathrm{e} 20$ & - \\
\hline$\sigma_{t}$ & $0.96889 \mathrm{e} 20$ & $0.33562 \mathrm{e} 20$ & $\mathrm{MPa}$ \\
\hline Obj. Funct. & $28,804.6$ & $9,449.82$ & - \\
\hline
\end{tabular}


Table 15 show the initial guess material constants values used by MACHO during the optimization process for 304 stainless steel low cycle fatigue, and the resultant optimized material constants values which were used for the simulation and exercise of the proposed hybrid model V1.

Table 15: Stainless Steel 304 Fatigue Material Constants for hybrid model V1

\begin{tabular}{cccc}
\hline $\begin{array}{c}\text { Material } \\
\text { constant }\end{array}$ & $\begin{array}{c}\text { Initial } \\
\text { guess } \\
\text { value }\end{array}$ & $\begin{array}{c}\text { Optimized } \\
\text { value }\end{array}$ & Units \\
\hline $\boldsymbol{B}$ & 256 & $0.44225 \mathrm{e}-1$ & $\mathrm{Sec}^{-1}$ \\
\hline $\boldsymbol{n}$ & 1 & 2.0033 & - \\
\hline $\boldsymbol{H}_{\boldsymbol{1}}$ & 5823 & 6608.6 & - \\
\hline $\boldsymbol{H}_{\boldsymbol{2}}$ & 159 & 1721.1 & $\mathrm{MPa}$ \\
\hline $\boldsymbol{S}_{\boldsymbol{1}}$ & 8523 & $0.19465 \mathrm{e}-9$ & - \\
\hline $\boldsymbol{M}$ & 1523 & $0.56284 \mathrm{e} 20$ & - \\
\hline$\phi$ & 1 & $0.17482 \mathrm{e} 20$ & - \\
\hline$\sigma_{t}$ & 7456 & $0.38283 \mathrm{e} 20$ & $\mathrm{MPa}$ \\
\hline Obj. Funct. & $270,930.8$ & $3,606.76$ & - \\
\hline
\end{tabular}

The proposed hybrid model V1 was exercised using the FEMCREEP feature of MACHO to perform numerical finite element (FE) simulations of creep and low cycle fatigue at multiple stress and strain amplitude levels, while at elevated temperature levels. The numerical simulation results are compared to experimental data in Figure 27-Figure 30. Figure 27 and Figure 28 shows the creep results of Hastelloy $\mathrm{X}$ at $950^{\circ} \mathrm{C}$ at different stress levels (35 Mpa. $30 \mathrm{MPa}, 20 \mathrm{MPa}, 18$ $\mathrm{MPa}, 16 \mathrm{MPa}$ ). In the other hand, Figure 29 and Figure 30 shows the low cycle fatigue results of 304 Stainless Steel at $600^{\circ} \mathrm{C}$, at strain amplitudes $(\Delta \varepsilon)$ of 0.005 and 0.007 .

In Figure 27 the numerical simulation results are compared to experimental data at $35 \mathrm{MPa}$ and $30 \mathrm{MPa}$. At his stress levels it can be observed how the proposed hybrid model does not performed a good prediction of the inelastic behavior of Hastelloy X. The hybrid model is under 
predicting the inelastic behavior by magnitudes of $0.7 \%$ creep strain. Figure 28 in the other hand, shows the creep results of Hastelloy $\mathrm{X}$ at lower stress levels $20 \mathrm{MPa}, 18 \mathrm{MPa}$ and $16 \mathrm{MPa}$. It can be observed an overall good correspondence between numerical simulation results and experimental data. At $20 \mathrm{MPa}$ the highest correspondence is exhibited, at this stress level the hybrid model is fully predicting the three creep stages. At $18 \mathrm{MPa}$ the accuracy of the model decreased but it still follows the tertiary creep path; however, the rupture creep strain is not accurately predicted by the model. At $16 \mathrm{MPa}$ the model is predicting tertiary creep; however the model is over predicting the creep strain. The rupture creep strain is over predicted by the model by magnitude of $0.1 \%$ creep strain. A step can be observed at low time (hours), this step is attributed to the transition from secondary to tertiary creep. An option to mitigate this transition effect is the use of an evolutionary drag stress rate equation. This evolutionary drag stress rate equation will allow a smoother transition between creep stages.

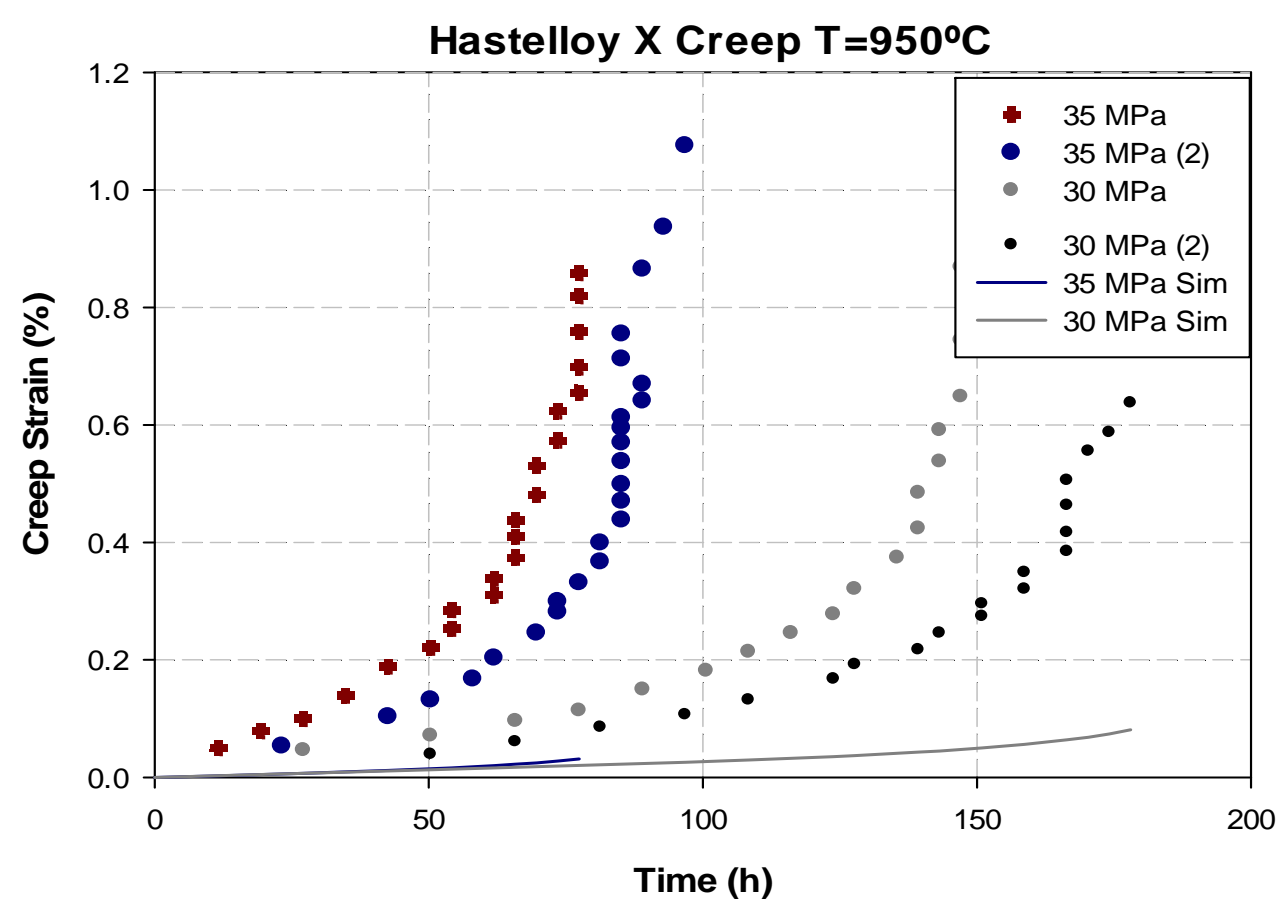

Figure 27: Hastelloy X creep experimental data vs. Hybrid model V1 simulated data 


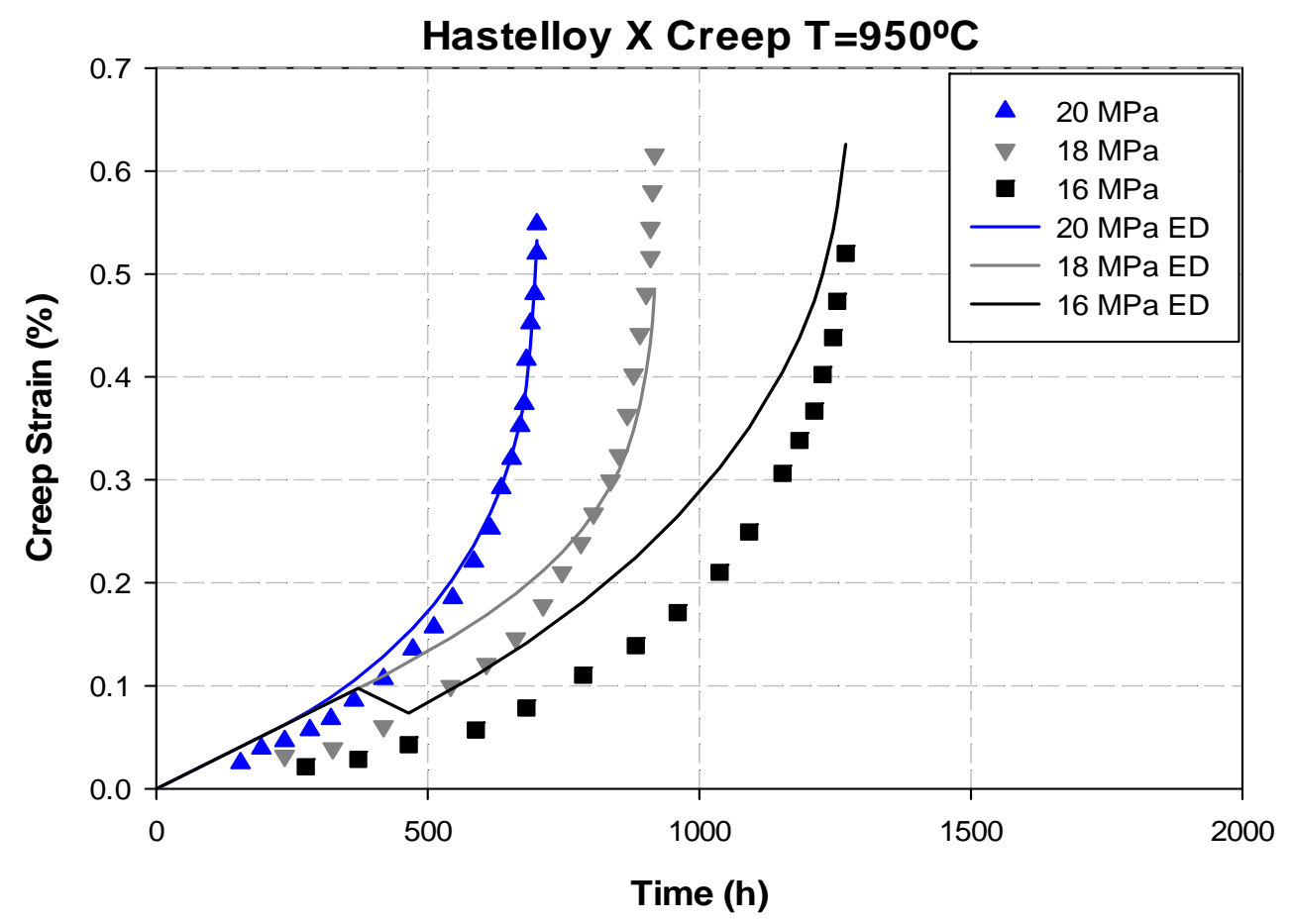

Figure 28: Hastelloy X creep experimental data vs. Hybrid model V1 simulated data

Figure 29 shows the numerical simulation results against the experimental data of low cycle fatigue at strain amplitude of 0.005 . It can be observed how there is a good overall correspondence between the curves at top stress levels, meaning that the hybrid model is successfully predicting the maximum hardening of the material. However, at the lower stress point or maximum softening point, the hybrid model is under predicting it. The hardening behavior of the material is well predicted by the model, this can be observed in the top curve of the hysteresis loop (from the lower to the higher stress point). The softening of the material is not predicted as well as the hardening section of the hysteresis loop. Figure 30 shows the numerical simulation results vs. experimental data at strain amplitude of 0.007. An overall good correspondence between the data can be observed. However at the maximum stress level or maximum hardening point, it can be observed how the hybrid model is over predicting the stress 
level. At the lower stress point or maximum softening point, it can be observed how the hybrid model is under predicting the stress level. At this strain amplitude in can be observed how the softening section of this hysteresis loop is better predicted compared to the previous hysteresis loop. This indicates that the softening terms of the model requires further improvement to successfully predict the softening behavior. The incorporated damage term, does not exhibited any effect in the low cycle fatigue simulations.

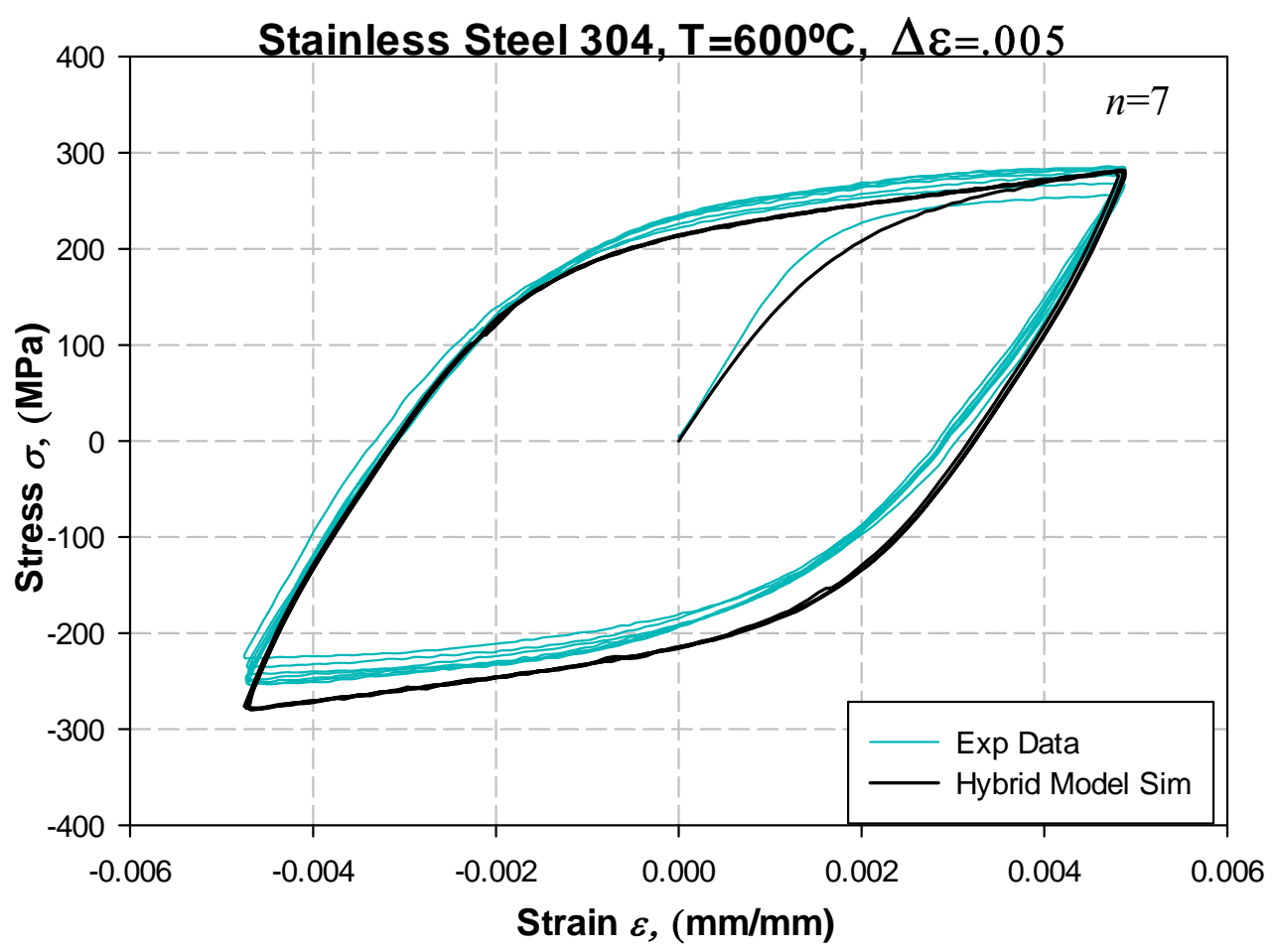

Figure 29: Stainless Steel 304 experimental data vs. Hybrid model V1 simulated data. 


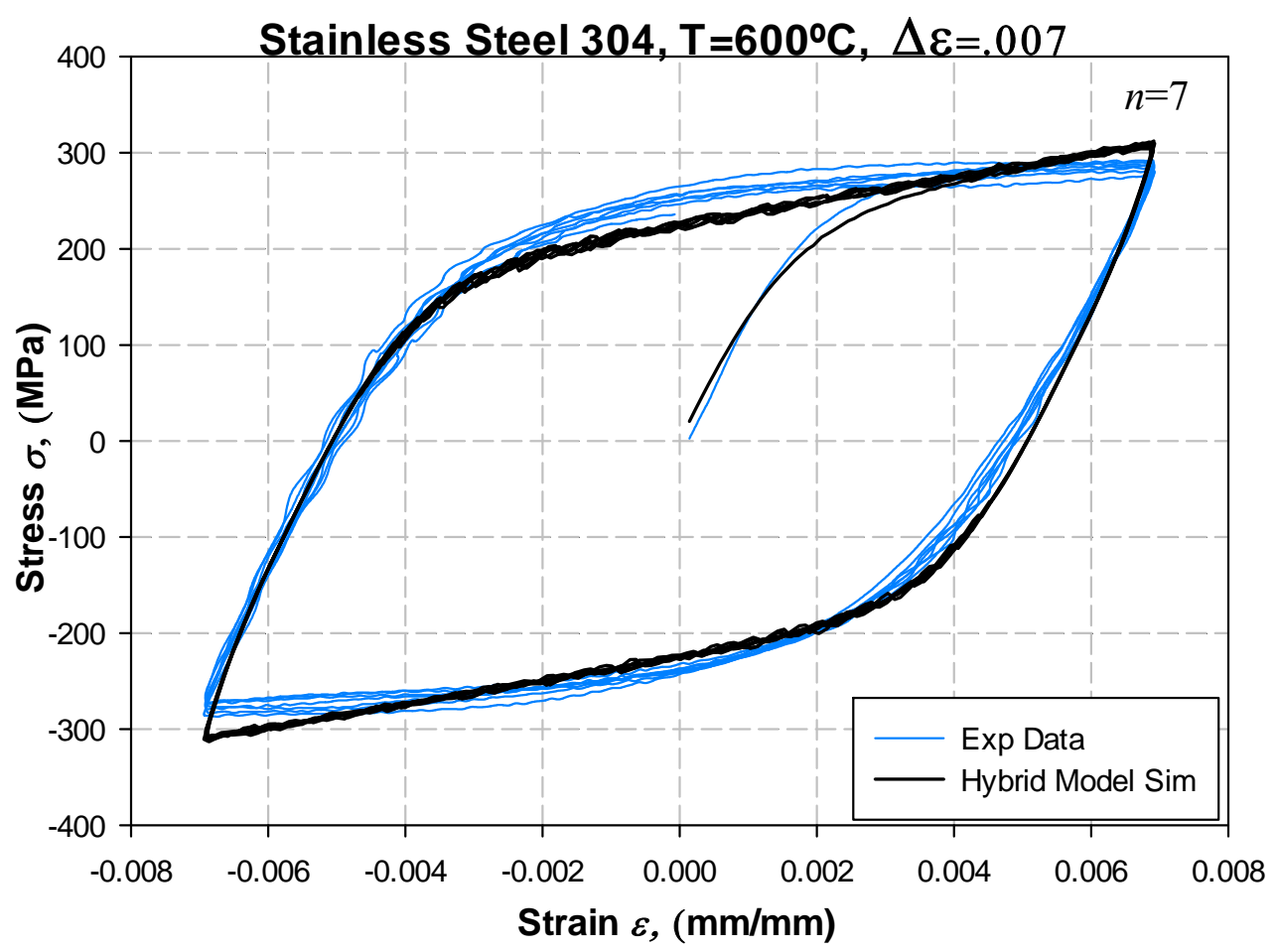

Figure 30: Stainless Steel 304 experimental data vs. Hybrid model V1 simulated data.

To establish a numerical or quantitative evaluation on the behavior the hybrid model with respect to the experimental data, the mean percentage error (MPE) and the coefficient of determination $\left(R^{2}\right)$ were calculated. The mean percentage error (MPE) was calculated between the experimental data and the numerical simulation data points for each data point at each stress level for Hastelloy X. The results are presented in Table 16. Table 17 presents the MPE between the experimental data and numerical simulation results of stainless steel 304 subjected to low cycle fatigue.

From Table 16 it can be observed how the model exhibited a higher accuracy at low stress levels $(\sigma \leq 20 \mathrm{MPa})$ simulating the creep behavior of Hastelloy X. At this stress levels the MPE is smaller compared to higher stress levels. Similarly considering the coefficient of determination $\left(R^{2}\right)$, the higher accuracy of the model is present at low stress levels where the coefficient of 
determination is closer to unity. The highest accuracy of the model is exhibited at $20 \mathrm{MPa}$ where the lowest MPE and highest coefficient of determination are present. This higher accuracy at an intermediate stress level (intermediate value of the considered stress levels) is expected since the material constants were optimized using the seven experimental data sets simultaneously. Therefore, MACHO is looking for optimized constants that would fit the experimental data in average, and $20 \mathrm{MPa}$ is the middle or average stress value. Moreover, the higher overall accuracy is present at low stress levels where the experimental time is larger; therefore, resulting in a higher weight or higher importance automatically assigned to this low stress levels.

Table 16: Mean percentage error (MPE) and coefficient of determination $\left(R^{2}\right)$ of Hastelloy X creep simulations hybrid model V1

\begin{tabular}{ccc}
\hline Stress Level & MPE (\%) & $\boldsymbol{R}^{\mathbf{2}}$ \\
\hline $35 \mathrm{MPa}$ & 90.38 & 0.21 \\
\hline $35 \mathrm{MPa}(2)$ & 57.8501 & 0.5009 \\
\hline $30 \mathrm{MPa}$ & 83.051 & 0.12 \\
\hline $30 \mathrm{MPa}(2)$ & 77.23867 & 0.15 \\
\hline $20 \mathrm{MPa}$ & 13.93849 & 0.991517 \\
\hline $18 \mathrm{MPa}$ & 27.7065 & 0.892781 \\
\hline $16 \mathrm{MPa}$ & 65.158 & 0.745854 \\
\hline
\end{tabular}

From table Table 17 it can be observed how the best accuracy of the model predicting low cycle fatigue behavior of 304 stainless steel is at high strain amplitudes. At 0.007 strain amplitude the higher accuracy is presented since the lower MPE and the highest coefficient of determination were determined. This meets the visual conclusion discussed above, where it was 
discussed how the hybrid model better predicts the softening of the hysteresis loop at 0.007 strain amplitude.

Table 17: Mean percentage error (MPE) and coefficient of determination $\left(R^{2}\right)$ of 304 stainless steel data low cycle fatigue simulations hybrid model V1

\begin{tabular}{ccc}
\hline Strain Amplitude & MPE & $\boldsymbol{R}^{\mathbf{2}}$ \\
\hline 0.005 & 42.344 & 0.9856 \\
\hline 0.007 & 20.334 & 0.9934 \\
\hline
\end{tabular}




\subsection{HYBRID MODEL V2}

Based on the hybrid model V1 constitutive equation and the previously discussed results an improved hybrid model V2 is presented. Major modifications were performed to the drag stress equation, it was modified to be an evolutionary drag stress rate equation where hardening and softening terms were added. The hybrid model V2 consists of ten material constants, an five state variables (inelastic strain rate, accumulated inelastic strain rate, rest stress, drag stress, and damage). Hybrid model V2 is function for isothermal tests, and it is capable of predicting monotonic tension, low cycle fatigue, primary, secondary and tertiary creep.

\subsubsection{MODEL DEVELOPMENT}

No modification were performed in the inelastic strain rate, damage rate and rest stress constitutive equation proposed in hybrid model V1, equations presented in Eq. (39)- Eq. (43) .

$$
\begin{gathered}
\dot{\omega}=\frac{M[1-\exp (-\phi]}{\phi} \sinh \left(\frac{\sigma}{\sigma_{t}}\right)^{3} \exp (\phi \omega) \\
\dot{R}=H_{1} \dot{\varepsilon}-S_{1} \dot{\varepsilon}_{p}|R| \\
\dot{\varepsilon}_{p}=|\dot{\varepsilon}| \\
\dot{\varepsilon}=B \sinh \left(\frac{|\sigma-R|}{D}\right)^{n} \operatorname{sgn}(\sigma-R) \exp \left(\lambda \omega^{\frac{3}{2}}\right) \\
\lambda=\ln \left(\frac{\dot{\varepsilon}_{\text {max }}}{\dot{\varepsilon}_{\text {min }}}\right)
\end{gathered}
$$


Based on Krieg, Swearenen and Rohde's (KSR) viscoplastic model [40], major modifications were performed in the drag stress equation. It was modified to be an evolutionary equation dependent of time; thus, the resultant drag stress equation is actually a drag stress rate equation presented in Eq.(44). It follows a basic hardening-softening format. The hardening term is dependent of the inelastic strain rate and the material constant $H_{2}$, which is responsible of the drag stress hardening due to the presence of an inelastic strain rate. The recovery or softening is not dependent of the inelastic strain rate or accumulated inelastic strain rate. Material constant $S_{2}$ and $S_{3}$ are responsible of the amount of hardening of the drag stress rate, while the material constant $n$ controls the intensity of the drag stress history impact in the softening term. The drag stress $D$ is used to account for the drag stress history, and the time step $\Delta \varepsilon$ scales the softening behavior as a function of time.

$$
\dot{D}=H_{2} \dot{\varepsilon}-S_{2} \Delta t\left(D-S_{3}\right)^{n}
$$

\subsubsection{EXERCISE OF HYBRID MODEL V2}

Table 18 shows the initial guess values used by MACHO for the optimization process and the optimized material constants for Hastelloy X creep. From this table it can be observed how before and after the values of the material constants $S_{2}$ and $S_{3}$ are equal to zero. Since these material constants are used as multipliers of other state variables, their zero value deactivate the softening term of the drag stress rate equation. Therefore, it can be concluded that the hybrid model V2 only requires the use of eight material constants to calculate the creep of Hastelloy X. 
Table 18: Hastelloy X creep material constants for hybrid model V2

\begin{tabular}{cccc}
\hline Material & Initial Guess & Optimized & Units \\
Constant & Value & Value & \\
\hline $\boldsymbol{B}$ & $0.21739 \mathrm{e}-2$ & $0.2022 \mathrm{e}-2$ & $\mathrm{Sec}^{-1}$ \\
\hline $\boldsymbol{n}$ & $0.22677 \mathrm{e}-2$ & $0.21834 \mathrm{e}-2$ & - \\
\hline $\boldsymbol{H}_{\boldsymbol{1}}$ & $0.62641 \mathrm{e} 20$ & $0.1 \mathrm{e} 21$ & - \\
\hline $\boldsymbol{H}_{2}$ & $0.60238 \mathrm{e} 17$ & 12,207 & $\mathrm{MPa}$ \\
\hline $\boldsymbol{S}_{\boldsymbol{1}}$ & $0.60211 \mathrm{e} 20$ & $0.1 \mathrm{e} 21$ & - \\
\hline $\boldsymbol{M}$ & $0.1324 \mathrm{e} 20$ & $0.98713 \mathrm{e} 20$ & - \\
\hline $\boldsymbol{\phi}$ & $0.82894 \mathrm{e} 20$ & $0.85562 \mathrm{e} 20$ & $\mathrm{MPa}$ \\
\hline $\boldsymbol{\sigma}_{\boldsymbol{t}}$ & $0.56998 \mathrm{e} 20$ & $0.93381 \mathrm{e} 20$ & $\mathrm{Sec}$ \\
\hline $\boldsymbol{S}_{2}$ & 0 & 0 & $\mathrm{MPa}$ \\
\hline $\boldsymbol{S}_{3}$ & 0 & 0 & \\
\hline Obj. Funct. & $2,487.44$ & & \\
\hline
\end{tabular}

Table 19 show the initial guess material constants values used b MACHO during the optimization process for 304 stainless steel low cycle fatigue, and the resultant optimized material constants values which were used for the simulation and exercise of the proposed hybrid model V2. From this table it can be observed that the material constant $n$ which is an exponent in the inelastic strain rate equation and an exponent in the drag stress rate equation is equal to unity, before and after the optimization process. Therefore, this indicates that hybrid model does not require the use of the material constant $n$; thus, only nine material constants are required to model low cycle fatigue using hybrid model V2. 
Table 19: 304 Stainless Steel Fatigue material constants for hybrid model V2

\begin{tabular}{|c|c|c|c|}
\hline $\begin{array}{l}\text { Material } \\
\text { Constant }\end{array}$ & $\begin{array}{c}\text { Initial Guess } \\
\text { Value }\end{array}$ & $\begin{array}{c}\text { Optimized } \\
\text { Value }\end{array}$ & Units \\
\hline $\boldsymbol{B}$ & $0.92241 \mathrm{e}-3$ & $0.16683 e-3$ & $\mathrm{Sec}^{-1}$ \\
\hline$n$ & 1.0 & 1.0 & - \\
\hline$H_{1}$ & $5,741.1$ & $6,591.9$ & - \\
\hline $\mathrm{H}_{2}$ & $0.2021 \mathrm{e} 6$ & 30,301 & $\mathrm{MPa}$ \\
\hline$S_{1}$ & 0.59532 & 0.64032 & - \\
\hline$M$ & 79.393 & 34.869 & - \\
\hline$\varnothing$ & $0.7582 \mathrm{e} 20$ & $0.18069 \mathrm{e} 20$ & - \\
\hline$\sigma_{t}$ & $0.37511 \mathrm{e} 20$ & $0.5766 \mathrm{e} 20$ & $\mathrm{MPa}$ \\
\hline$S_{2}$ & $0.41836 \mathrm{e} 19$ & $0.29356 \mathrm{e} 20$ & $\mathrm{Sec}^{-2}$ \\
\hline$S_{3}$ & 294.45 & 103.29 & $\mathrm{MPa}$ \\
\hline Obj. Funct. & $3,102.31$ & $2,741.23$ & - \\
\hline
\end{tabular}




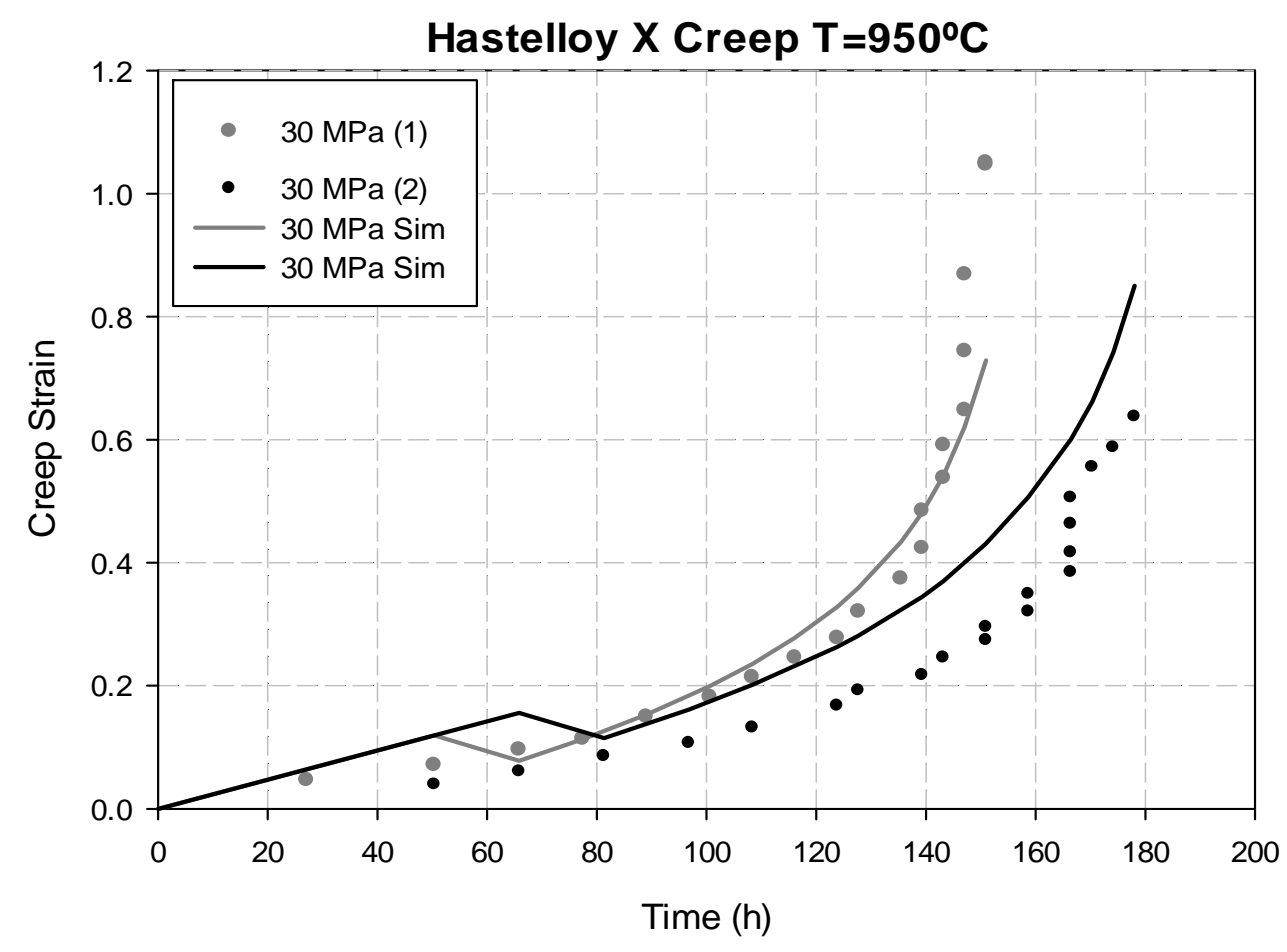

Figure 31 shows the experimental data and numerical simulation results for creep behavior of Hastelloy X at $35 \mathrm{MPa}$ using hybrid model V2. It can be observed how the numerical simulation results are following the tertiary creep regime with some discrepancies. The hybrid model V2 is under predicting the tertiary creep regime for this stress level. However the secondary creep regime is well predicted in both simulation results. Figure 32 shows the experimental data and the numerical simulation results for creep behavior of Hastelloy X at $30 \mathrm{MPa}$ using hybrid model V2. 


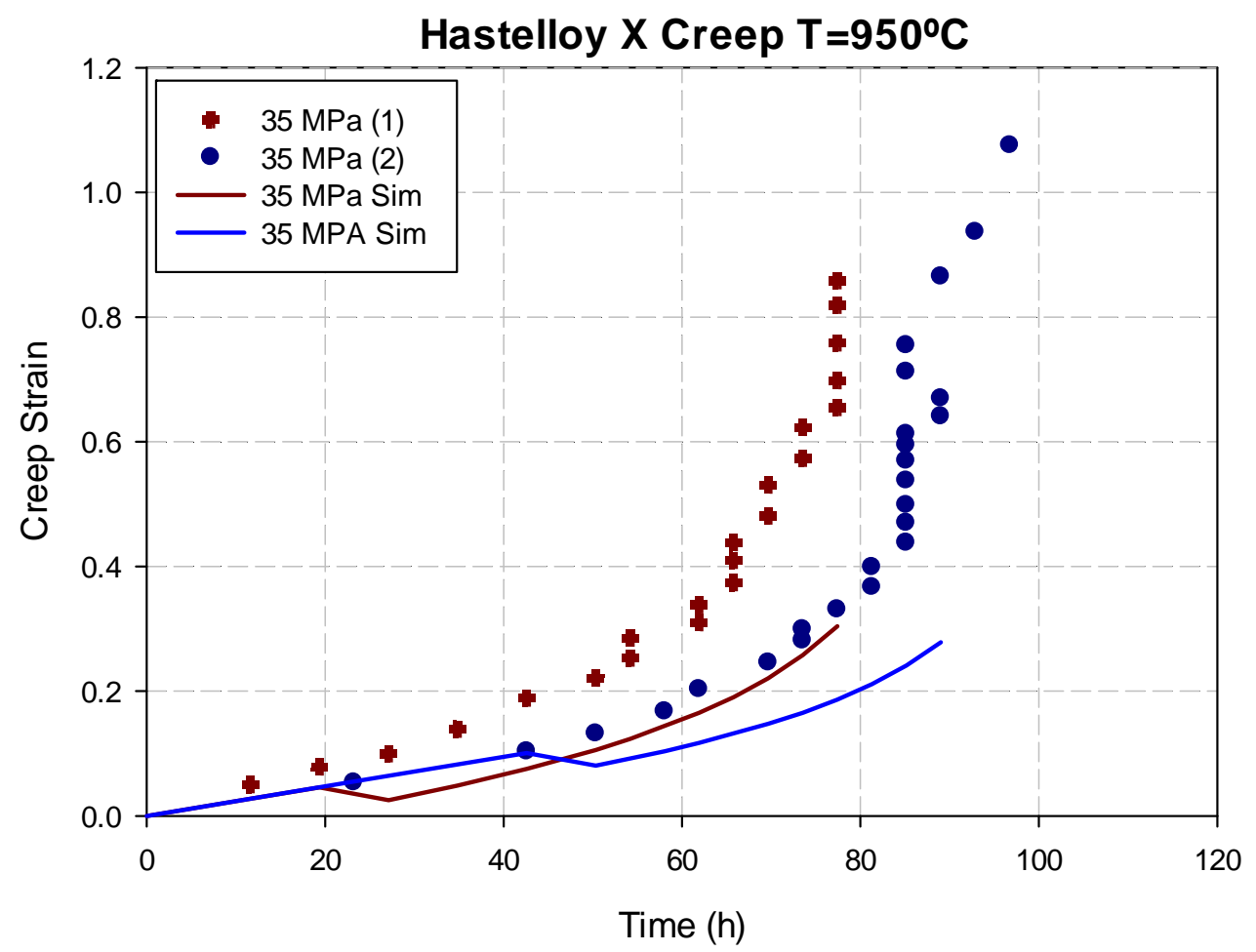

Figure 31: Hastelloy X creep experimental data vs. Hybrid model V2 simulated data

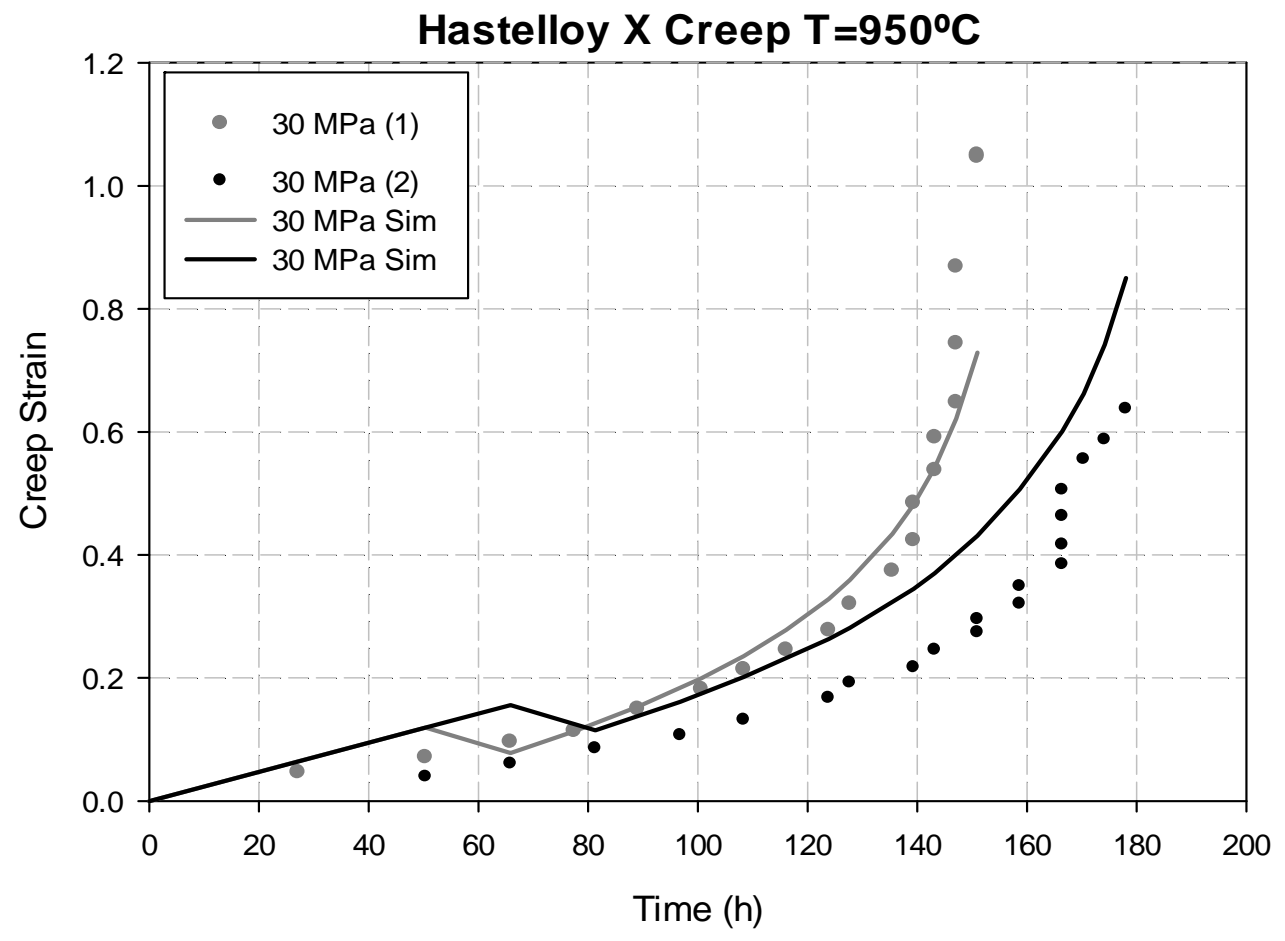

Figure 32: Hastelloy X creep experimental data vs. Hybrid model V2 simulated data 
A good correspondence between the experimental and simulation data can be observed, since the simulation data is better following the tertiary creep regime it both cases. The first test (gray) is exhibiting the highest accuracy. The second test (black) is over predicting the creep behavior; however it is following the experimental data path by predicting tertiary creep. No other stress levels were simulated since the current stage of the constitutive model is not allowing the calculation of material constants for them, even when they are optimized individually. This is attributed to the current equations format. Just as demonstrated in Table 18 constant $S_{2}$ and $S_{3}$ are equal to zero for creep prediction; therefore the softening term of the drag stress rate equation is fully deactivated. Thus, the drag stress rate equation is function of the inelastic strain rate and the hardening constant $\mathrm{H}_{2}$. While being function of these terms, it means that the drag stress is slightly changing throughout the simulation since it is dependent on the time step size (due to the strain rate). However the issue appears when the drag stress rate is converted to a drag stress to be saved as a state variable. The drag stress rate is multiplied by the time step increment (which converts it into a drag stress) and then it is added to the drag stress value from the previous step. Therefore the drag stress (state variable) is constantly increasing, reaching saturation at an early stage and causing the optimization software to crash for lower stress levels. This is the reason why Walker model does not use an evolutionary drag stress equation.

Figure 33 shows the experimental data against the numerical simulation results produced by the hybrid model V2 during low cycle fatigue of 304 stainless steel at a strain amplitude of 0.005. From Figure 33 it can be observed how the hardening behavior is well predicted by the hybrid model V2, except at the maximum stress point, were it is over predicted by the proposed model. The softening behavior is not as well predicted as the hardening behavior; however, there is a good correspondence at the beginning of the hardening behavior and at the very end where 
the minimum stress point is slightly under predicted by the proposed model. However, the best correspondence between experimental data and numerical simulation data is generated by hybrid model V2, when compared to the previous versions.

Figure 34 shows the experimental data against the numerical simulation data generated by the hybrid model at a strain amplitude of 0.0007 , for 304 stainless steel. From this figure, it can be observed how there is a good correspondence between the numerical simulation results and the experimental data during the hardening section of the hysteresis loop. However, at the maximum stress point the proposed model is over predicting the stress level. The softening behavior is overall well predicted however, there are some discrepancies in the middle section where the hybrid model is overproduction the behavior. The minimum stress point is well predicted by the proposed model.

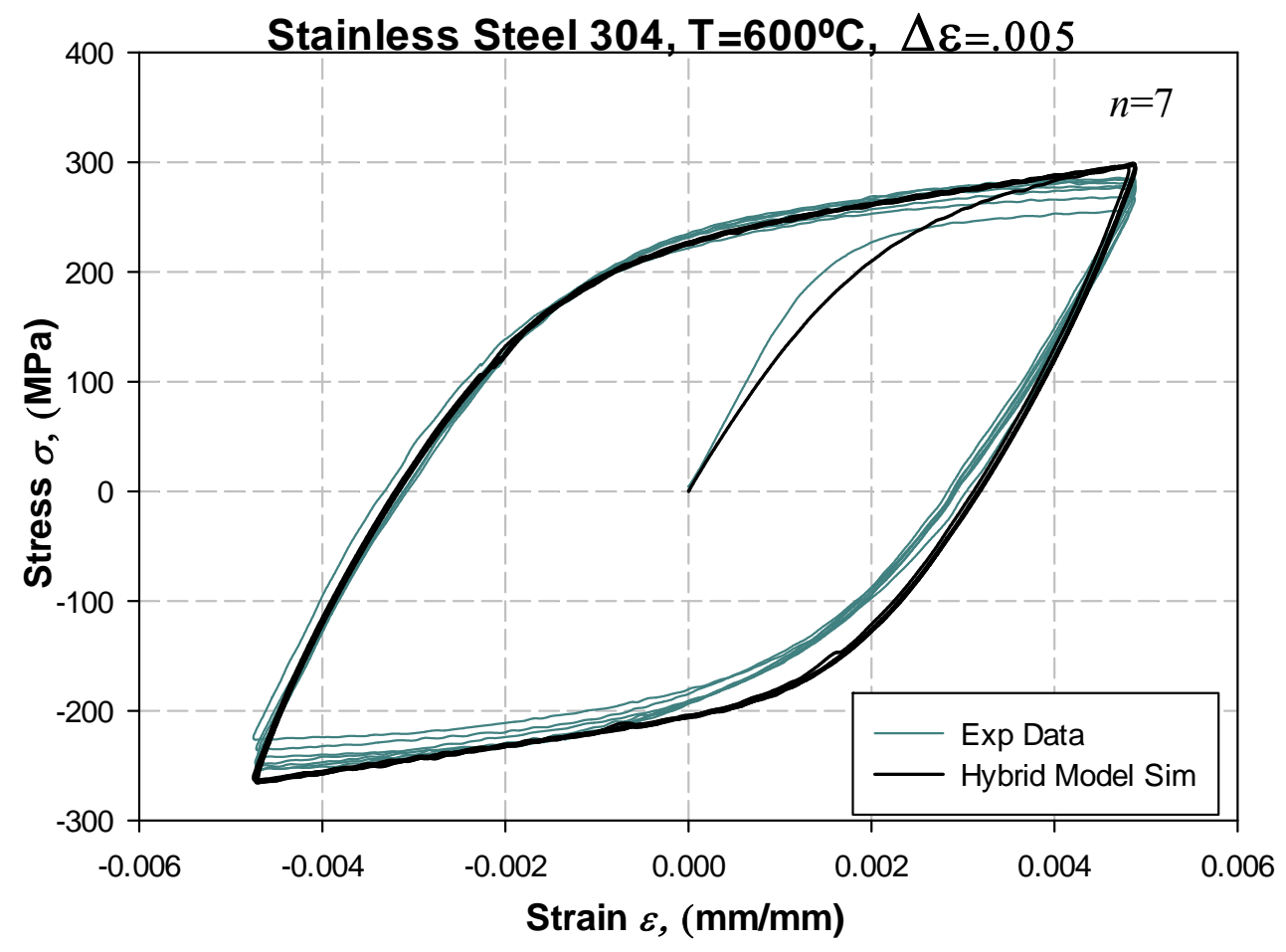

Figure 33: Stainless Steel 304 experimental data vs. Hybrid model V2 simulated data. 


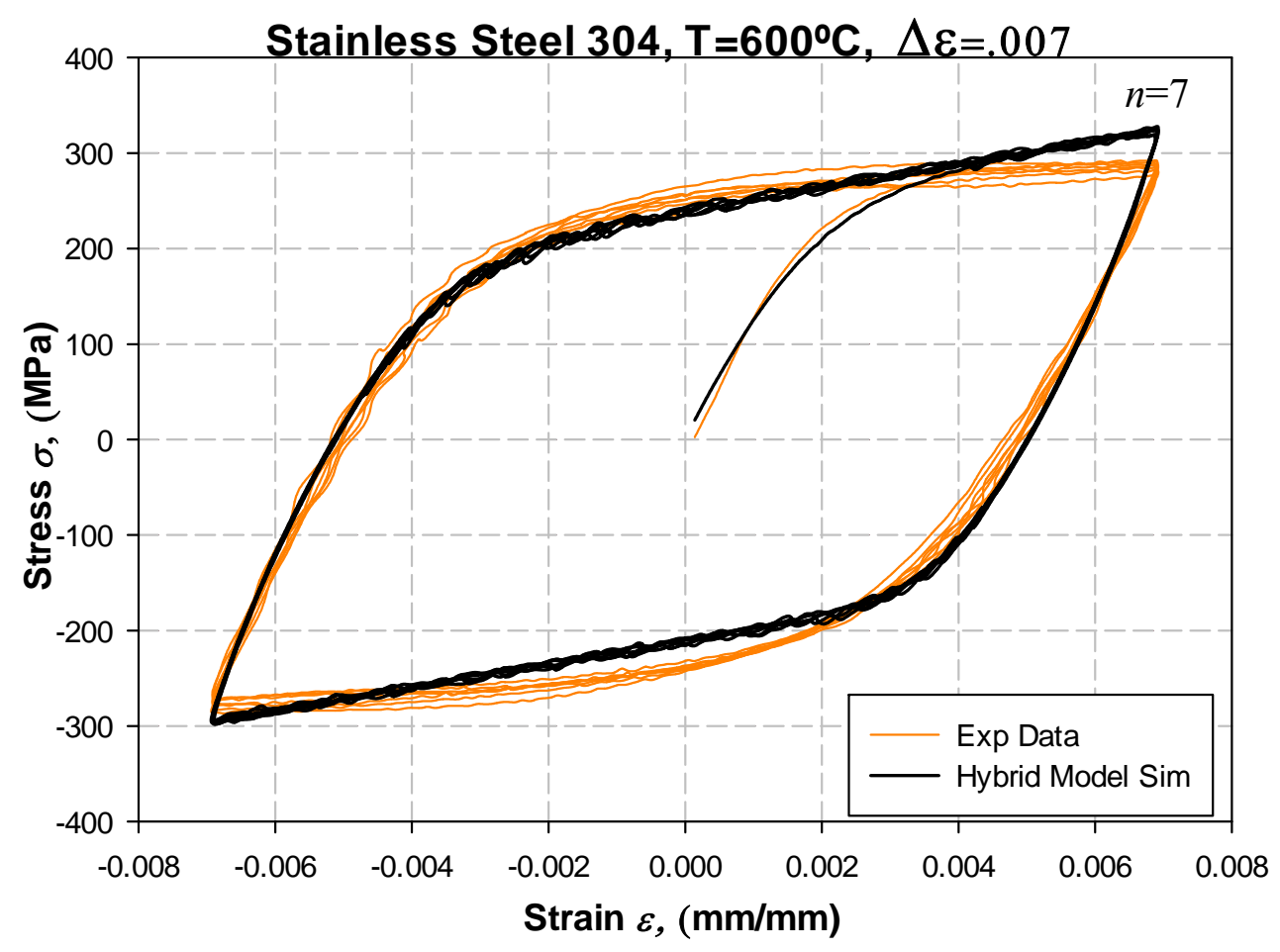

Figure 34: Stainless Steel 304 experimental data vs. Hybrid model V2 simulated data. 
Figure 35 and Figure 36 show the stress amplitude with respect to the number of cycles at strain amplitudes of 0.005 and 0.007 , respectively. In this figures the previously predicted stress amplitude of hybrid model V2 is compared to experimental data. Figure 35 shoes the predicted stress amplitude with respect to the number of cycles and it is compared to experimental data at strain amplitude of 0.005. from this figure it can be observed how the hybrid model is over predicting the stress levels at the hardening points during the first couple of cycles, however as the number of cycles increases and the material hardens the predicted stress levels is exhibiting a close correspondence with experimental data. on the other hand, in the softening region the hybrid model is under predicting the stress levels during the first couple of cycles, and as the material hardening the correspondence between data increases. Figure 36 compares the experimental data to predicted data at strain amplitude of 0.007 . From this figure it can be observed how the hybrid model is over predicting the stress level in the hardening region. Within the softening region the hybrid model is presenting a higher correspondence with experimental data, the stress level is accurately predicting during the first couple of cycles and as the number of cycles increases the model is under predicting it. From this image it can be concluded that the hybrid model is not evolving as the experimental data, meaning that the hardening and/or softening predicted by the hybrid model is being predicted as an almost constant value. 


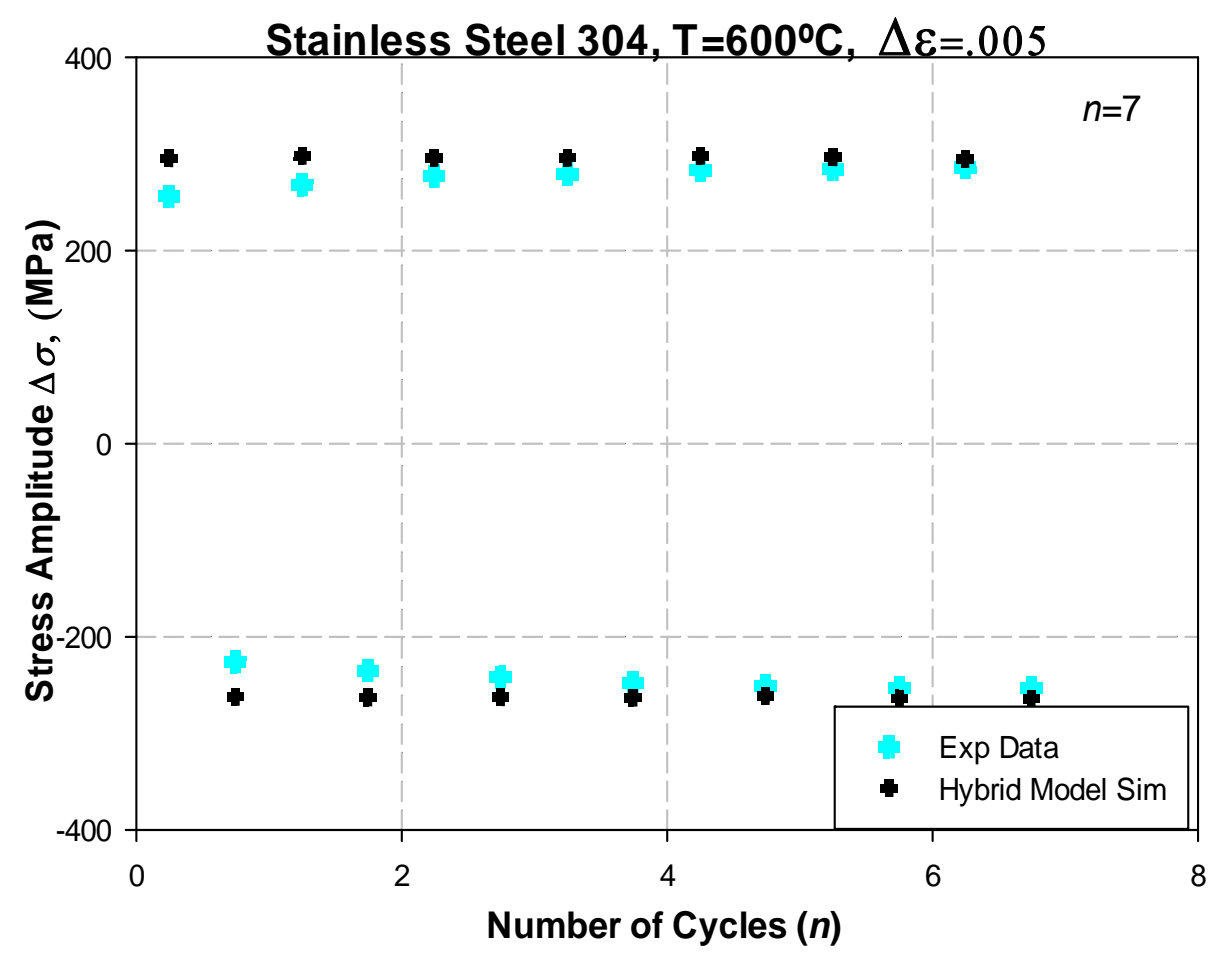

Figure 35: Comparison of stress amplitude and number of cycles at $\Delta \varepsilon=0.005$

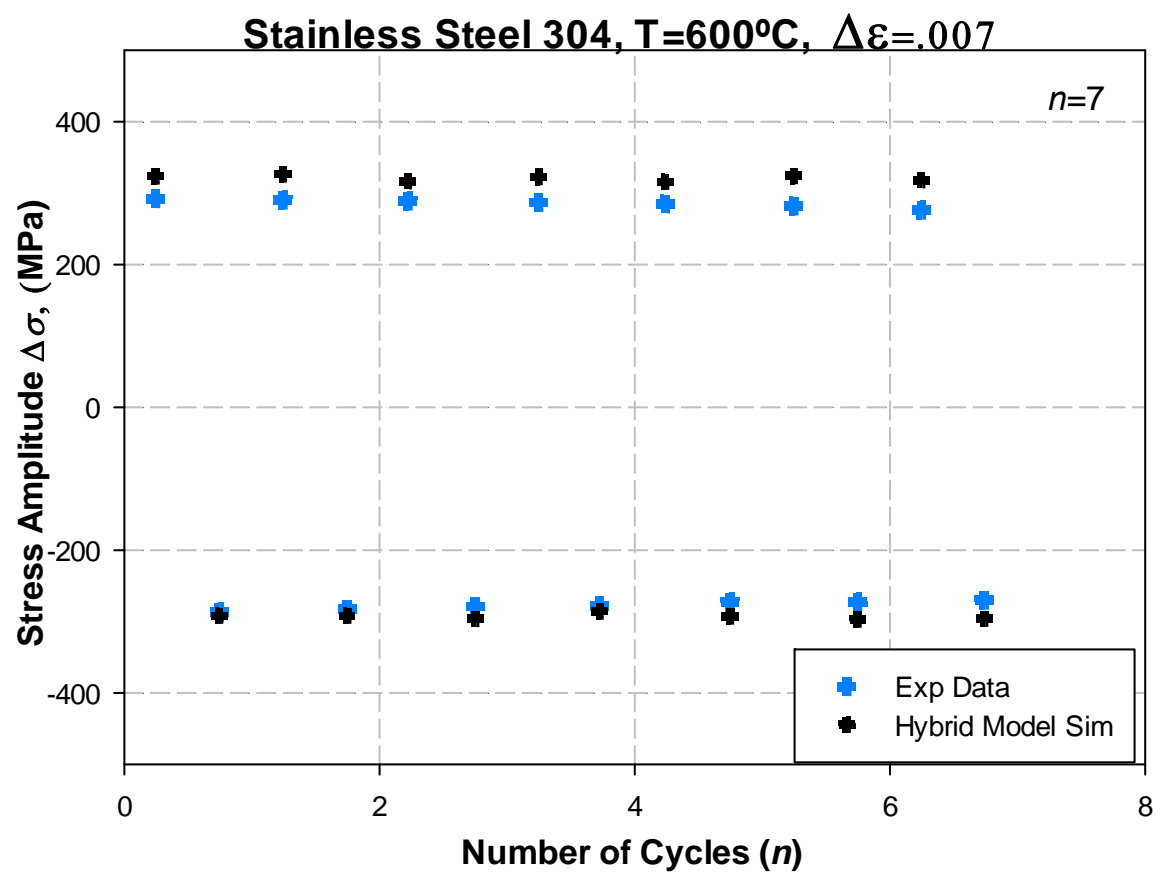

Figure 36: Comparison of stress amplitude and number of cycles at $\Delta \varepsilon=0.007$ 
From Table 20 it can be observed how the accuracy of the hybrid model V2 is higher than hybrid model V1 for high stress levels. The highest accuracy was at the first test at $30 \mathrm{MPa}$, and the lowest accuracy was at the second test at $35 \mathrm{MPa}$ (2). This difference in accuracy is attributed to the importance or weight that MACHO automatically assigns to the experimental data set with the longest duration time; however, since the average is used that is the reason why the best fit is found in the data set with the average time value.

Table 20: Mean percentage error (MPE) and coefficient of determination $\left(R^{2}\right)$ of Hastelloy X data creep simulations hybrid model V2

\begin{tabular}{ccc}
\hline Stress Level & MPE (\%) & $\boldsymbol{R}^{\mathbf{2}}$ \\
\hline $35 \mathrm{MPa}$ & 53.31072 & 0.2015 \\
\hline $35 \mathrm{MPa}(2)$ & 92.27 & 0.0708 \\
\hline $30 \mathrm{MPa}$ & 16.203 & 0.85486 \\
\hline $30 \mathrm{MPa}(2)$ & 54.5937 & 0.491694 \\
\hline $20 \mathrm{MPa}$ & NAN & NAN \\
\hline $18 \mathrm{MPa}$ & NAN & NAN \\
\hline $16 \mathrm{MPa}$ & NAN & NAN \\
\hline
\end{tabular}


From Table 21 it can be observed how the best accuracy of the model predicting low cycle fatigue behavior of 304 stainless steel is at high strain amplitudes. At 0.007 strain amplitude the higher accuracy is presented since the lower MPE and the highest coefficient of determination were determined. However, based on the results of hybrid model V1 there was a great improvement in the prediction of the low cycle fatigue behavior at a lower strain amplitude (0.005). this improvement is due to a better prediction of the softening behavior, which is attributed to the improvement performed in the drag stress rate equation. Based on the results of hybrid model V1, there was a small increment (about 0.3\% MPE) this is attributed to the optimization process which is optimizing constants for both strain amplitudes simultaneously.

Table 21: Mean percentage error (MPE) and coefficient of determination $\left(R^{2}\right)$ of 304 stainless steel data low cycle fatigue simulations hybrid model V2

\begin{tabular}{ccc}
\hline Strain Amplitude & MPE & $\boldsymbol{R}^{\mathbf{2}}$ \\
\hline 0.005 & 29.542 & 0.992655 \\
\hline 0.007 & 20.586 & 0.993448 \\
\hline
\end{tabular}




\section{CHAPTER 7: CONCLUSION \& FUTURE WORK}

\subsection{CONCLUSIONS}

The following conclusions can be formulated from the exercise of Miller and Walker Constitutive models:

- By calculating materials constants with an effective systematic approach, Miller and Walker constitutive models can go beyond the designed limitations and model the three stages of creep when used at stresses $\sigma \geq 20$. When $\sigma<20 \mathrm{MPa}$ both models predict primary and secondary creep only.

- Both constitutive models can be used in non-isothermal conditions; however the total number of material constants required for each constitutive model will increase, especially for Walker model. The authors recommend the use of MACHO to optimize the temperature dependent material constants for both constitutive models.

- Walker model does not require the use of more material constants to simulate creep and fatigue behavior. MACHO simulations were performed in an effort to optimize all fourteen material constants and the results did not show a better correspondence with experimental data, this confirms Walker assumptions [56]. Therefore, it can be concluded that Walker constitutive equation can be simplified for creep and low cycle fatigue simulations, since some components are fully deactivated.

- The predictions for creep of both models show a good correspondence with experimental data at stresses above $20 \mathrm{MPa}$, where both models go beyond design limitations.

- At low stresses (below $20 \mathrm{MPA}$ ) significant deviations between numerical simulated data and experimental data is observed, especially at the tertiary creep regime. 
- According to the results presented in the present work for creep, Walker model exhibited a better accuracy at stresses above $18 \mathrm{MPa}$. Whereas at stresses below $18 \mathrm{MPa}$, the Miller model exhibited a higher accuracy.

- During low cycle fatigue, at a low strain amplitude Walker is considerably more accurate than Miller model. At a high strain amplitude both models exhibited a high accuracy, however Walker model is slightly more accurate.

- It has been proven that both viscoplastic models have the capabilities to model more than one alloy type.

- The functionality of MACHO to optimize material constants for multiple alloys has been proven.

- Considering the presented loading cases, the authors conclude Walker model to be a better model during isothermal test while using optimized material constants values.

The development of a novel hybrid viscoplastic model has been presented, explained and exercised for creep and low cycle fatigue at different stress levels and strain amplitudes. From the presented results the following conclusion can be formulated:

- The addition of the damage term to the basic hybrid model, leads to the prediction of the tertiary creep stage.

- The presence of the "step" in the transition region between secondary and tertiary creep is attributed to the interaction between the inelastic strain rate and the damage rate equation. Further testing is recommended to determine reason of the appearance of the "step".

- The use of a non-evolutionary drag stress equation, allows the successful prediction of tertiary creep at stresses equal or below $20 \mathrm{MPa}$, a good overall prediction of low cycle 
fatigue is generated. However, an evolutionary (non-constant) drag stress function should produce better prediction at high stress levels.

- From the creep simulations, it can be observed how the importance of having a similar weight or importance on each data set affects the material constant optimization, which consequently affects the model behavior. To mitigate this issue, each data set (stress level) should be optimized individually; however, different material constant values will result for each stress level.

- From the fatigue results it can be observed how the improvement of the hardening equations have led to improvements on the model predictions for creep and low cycle fatigue. 


\subsection{FUTURE WORK}

The following future work is suggested:

- Optimize material constants for each stress level individually, to mitigate the weight or importance issue associated with experimental time duration during creep.

- Modify MACHO to assign an equal importance level to each stress level, not mattering the test duration time.

- Test the life prediction capabilities of the incorporated damage term under creep conditions.

- Add a damage term that counts for damage caused by low cycle fatigue and accounts for life prediction.

- Investigate the impact of the rest and drag stresses in the damage evolution law.

- Further exercise the proposed hybrid model to validate its functionality for other alloys.

- Exercise the proposed hybrid model under different loading cases, such as: stress relaxation, fatigue with stress holds and fatigue with strain holds.

- Further improvement of the hardening equation to account for progressive hardening of the material. 


\section{REFERENCES}

[1] Totemeier, T. and Tian, H., 2006, "Creep-fatigue-environment interactions in INCONEL 617," Journal of Material science and Engineering, 468-470, pp. 81-87.

[2] Cabet, C., Carroll, L. and Wright, R., 2013, "Low Cycle Fatigue and Creep-Fatigue Behavior of Alloy 617 at High Temperature," Journal of Pressure Vessel Technology, 135, (2).

[3] Tachibana, Y. and Iyoku, T., 2004, "Structural design of high temperature metallic components," Journal of Nuclear Engineering and Design, 233, (1-3), pp. 261-272.

[4] Lee, H., Song, K., Kim, Y., Hong, S. and Park, H., 2011, "An Evaluation of Creep-Fatigue Damage for the Prototype Process Heat Exchanger of the NHDD Plant," Journal of Pressure Vessel Technology, 133, (5).

[5] Kurihara, R. and Ueda, S., 1987, "Study of Internal Pressure Creep Strength of Hastelloy X Cylindrical Specimen Containing an Axial Surface Notch,” Int. J. Pres. Ves. \& Piping, 30, pp. 37-56.

[6] Davis, C. B., Oh, C. H., Barner, R. B., Sherman, S. R., and Wilson, D. F., "ThermalHydraulic Analyses of Heat Transfer Fluid Requirements and Characteristics for Coupling A Hydrogen Production Plant to a High-Temperature Nuclear Reactor", INL/EXT-05-00453, Idaho National Laboratory, June 2005.

[7] Harvego, E. A., "Evaluation of Next Generation Nuclear Power Plant (NGNP) Intermediate Heat Exchanger (IHX) Operation Conditions", INL/EXT-06-11109, Idaho National Laboratory, April 2005.

[8] Swaminathan, B., Abuzaid, W., Sehitoglu, H. and Lambros, J., 2014, "Investigation using digital image correlation of Portevin-Le Chatelier in Hastelloy X under thermo-mechanical loading," International Journal of Plasticity.

[9] Misseijer, R. C. and Thabit, T. I., 1996, "Operational Evaluation of Patch Repaired Cobustion Gas Turbine Transition Pieces," Journal of Quality in Maintenance Engineering, 2, (4), pp. 59-70.

[10] ALSTOM Power, 2015, http://www.alstom.com/microsites/power/ 
[11] Kirka, M. M., Smith, D. J. and Neu, R. W., 2014, "Efficient Methodologies for Determining Temperature-Dependent Parameters of a Ni-Base Superalloy Crystal Viscoplasticity Model for Cyclic Loadings," Journal of Enginering Materials and Technology, 136.

[12] Konter, M. and Thumann, M., 2001, "Materials and Manufacturing of Advanced Industrial Gas Turbine Components," Journal of Materials Processing Technology, 117, pp. 386-390.

[13] Corman, J. C., 1996, "H Gas Turbine Combined Cycle Technology \& Development Status," Presented at the International Gas Turbine and Aeroengine Congress \& Exhibition Birmingham,UK.

[14] Honjo, T., et al., 1994, "Manufacturing and Mechanical Properties of a Large Size Alloy 706 Disk by ESR Process," Superalloys 718, 625, 706 and Various Derivatives, The Minerals Metals \& Materials Society.

[15] Kunkel, R. and Kollmann, F. G., 1997, "Identification of Constants of a Viscoplastic Constitutive Model for a Single Crystal Alloy," Acta Mechanica, 124, pp. 27-45.

[16] Markocsan, N., et al., 2007, "Low Thermal Condictivity Coating for Gas Turbine Applications," Journal of Thermal Spray Technology, 16, (4), pp. 498-505.

[17] Staroselsky, A., Martin, T. J. and Cassenti, B., 2015, "Transcient Thermal Analysis and Viscoplastic Damage Model for Life Prediction of Turbine Components," Journal of Enginering for Gas Turbines and Power, 137.

[18] Zhao, J. C., Larse, M. and Ravikumar, V., 2000, "Phase Precipitation and TimeTemperature-Transformation diagram of Hastelloy X," Material Science and Engineering, A293, pp. 112-119.

[19] ACT Independent Turbo Services, 2015, Photo Gallery, http://www.act-texas.com/areas-ofexcellence/combustion-repair/

[20] Schilke, P. W., Foster, A. D., Pepe, J. J., and Beltran, A. M., 1992, “Advanced Materials Propel Progress in Land-based Gas Turbines ," Advanced Materials and Processes, 141(4), pp. 22-30. 
[21] Lee, J. and Kuo, Y., 2006, "A Study on the Microstructure and Cyclic Oxidation Behavior of the Pack Aluminized Hastelloy X at $1100^{\circ} \mathrm{C}$," Surface \& Coating Technology, 201, pp. 3867-3871.

[22] Srivastava, S. K. and Klarstrom, D. L., 1990, "The LCF Behavior of Several Solid Solution Strengthened Alloys Used in Gas Turbine Engines," Presented at the Gas Turbine and Aeroengine Congress and Exposition, Brussels, Belgium.

[23] Tanner, D. W. J., Sun, W. and Hyde, T. H., 2011, "FE Analysis of a Notched Bar Under Thermomechanical Fatigue Using a Unified Viscoplasticity Model," Precedia Engineering, 10, pp. 1081-1086.

[24] Wikipedia, 2015, http://en.wikipedia.org/wiki/Turbine_engine_failure

[25] Owatonna, 2015, http://www.owatonnautilities.com/sites/Turbine\%20Blade2.jpg

[26] Almroth, P., et al., 2004, "Viscoplastic-plastic modeling of IN792," Computation Materials Science, 29, pp. 437-445.

[27] Stewart, C. M. and Gordon, A. P., 2009, "Modeling the Temperature Dependence of Tertiary Creep Damage of a Ni-Based Alloy," Journal of Pressure Vessel Technology, 131.

[28] Sakthivel, T., et al., 2011, "Effect of Temperature and strain rate on serrated flow behavior of Hastelloy X," Journal of Materials Science and Engineering, 534, pp. 580-587.

[29] Reed, C. R., 2008, The Superalloys: Fundamentals and Applications, Cambridge University Press, Cambridge, UK.

[30] Aghaie-Khafri, M. and Golarzi, N., 2007, "Forming behavior and workability of Hastelloy X superalloy during hot deformation," Journal of Materials Science \& Engineering A, 486, pp. 641-647.

[31] Kim, W., Yin, S., Ryu, W., Chang, J. and Kim, S., 2006, "Tension and creep design stresses of the "Hastelloy-X" alloy for high-temperature gas cooled reactors," Journal of Materials Science \& Engineering, 483-484, pp. 495-497. 
[32] Kim, W., Yin, S., Kim, Y. and Chang, J., 2008, "Creep characterization of a Ni-based Hastelloy-X alloy by using theta projection method," Journal of Engineering Fracture Mechanics, 75, pp. 4985-4995.

[33] Udoguchi, T. and Nakanishi, T., 1980, "Structural Behavior of a Welded Superalloy Cylinder with Internal Pressure in a High Temperature Environment," Int. J. Pres. Ves. \& Piping, 9, pp. 107-123.

[34] Miner, R.V. and Castelli, M.G., 1992, "Hardening Mechanisms in a Dynamic Strain Aging Alloy, Hastelloy X, during Isothermal and Thermomechanical Cyclic Deformation," International Journal of Fatigue, 14, (6), pp. 551-561.

[35] Aghaie-Khafri, M. and Golarzi. N., 2008, "Dynamic and Metadynamic Recrystallization of Hastelloy X Superalloy," Journal of Material Science, 43, pp. 3717-3724.

[36] Mehta, J. M. and Askew, J., 1997, "Future Material Needs for Low Emissions Gas Turbines," Presented at the International Gas Turbine \& Aeroengine Congress \& Exhibition, Orlando, Florida.

[37] Muktinutalapati, N. R., 2011, "Materials for Gas Turbines- An overview," Advances in Gas Turbine Technology, Dr. Ernesto Benini (Ed.).

[38] Aghaie-Khafri, M. and Golarzi. N., 2008, "Forming Behavior and Workability of Hastelloy X Superalloy During Hot Deformation," Materials Science and Engineering, A486, pp.641647.

[39] Krempl, E., 1974, “Cyclic Creep, an Interpretive Literature Survey," Welding Research Council Bulletin, 195.

[40] Chang, T. Y. and Thompson, R. L., 1984, “A Computer Program For Predicting Nonlinear Uniaxial Material Responses Using Viscoplastic Models," NASA Technical Memorandum 83675.

[41] Hartmann, G. and Kollmann, F.G., 1987, "A computational Comparison of the Inelastic Constitutive Models of Hart and Miller," Acta Mehcanica, 69, pp. 139-165

[42] Dombrovsky, L.A., 1992, "Incremental Constitutive Equations for Miller and BodnerPartom Viscoplastic Models," Computers \& Structures, 44, (5), pp. 1065-1072 
[43] Lindholm, U. S., Chan, K. S., Bodner, S. R., Weber, R. M., Walker, K. P. and Cassenti, B. N., 1984, "Constitutive Modeling for Isotropic Materials," NASA Report 174718, NASA.

[44] James, G. H., Imbrie, P. K., Hill, P. S., Allen, D. H. and Haisler, W. E., 1987, “An Experimental Comparison of Several Current Viscoplastic Constitutive Models at Elevated Temperature," Journal of Engineering Materials and Technology, 109, (2), pp. 130-139.

[45] L. A. Dombrovsky, "Incremental constitutive equations for Miller and Bodner-Partom viscoplastic models". Journal of Computers and Structures, Vol. 44, pp. 1065-1072, 1992.

[46] H. Alberg, "Material modelling for simulation of heat treatments". Licentiate Thesis, Department of Applied Physics and Mechanical Engineering Division of Computer Aided Design, 2003.

[47] J. L. Chaboche, "A review of some plasticity and viscoplasticity constitutive theories", International Journal of Plasticity, Vol. 24, pp. 1642-1693, 2008.

[48] M. C. Araujo, "Non-linear kinematic hardening model for multiaxial cyclic plasticity", Thesis submitted in The Department of Civil and Environmental Engineering at the Louisiana State University, 2002.

[49] R. D. Krieg, "Numerical integration of some new unified plasticity-creep formulations", Proc. Of 4th International Conference on Structural Mechanics in Reactor Technology, San Francisco, M6/b, 1977.

[50] T.Y. Chang and R.L. Thompson, “A computer program for predicting nonlinear uniaxial material responses using viscoplastic models”. NASA report, TM-83675, 1984.

[51] Miller, A. K, "A Unified Phenomenological Model for the Monotonic, Cyclic, and Creep deformation of Strongly Work-Hardening Materials," PhD dissertation, Stanford University Department of Materials Science and Engineering, 1975.

[52] Miller, A. K, “An Inelastic Constitutive Model for Monotonic, Cyclic, and Creep Deformation: Part II - Application to Type 304 Stainless Steel," Journal of Engineering Materials and Technology, Vol. 98, No. 2, 1976, pp. 106-113.

[53] Miller, A. K, "An Inelastic Constitutive Model for Monotonic, Cyclic, and Creep Deformation: Part I - Equations Development and Analytical Procedures," Journal of Engineering Materials and Technology, Vol. 98, No. 2, 1976, pp. 97-105. 
[54] Eleiche A. M., "Inelastic Deformation of Metals and Structures under Dynamic and Quasistatic cyclic loading" Final Technical Report, European Research Office of the U.S. Army, 1984.

[55] Varela, L., and Stewart, C. M., 2014, "An Inelastic Constitutive Model for Monotonic, Cyclic and Creep Deformation: Summary," 2014 4th Southwest Energy Science and Engineering Symposium, El Paso, Texas, March 22th, 2014.

[56] Walker, K. P., "Research and Development Program for Nonlinear Structural Modeling with Advanced Time-Temperature Dependent Constitutive Relationships," Final report NASA 165533, NASA.

[57] Hartmann, G., "Comparison of The Uniaxial Behavior of the Inelastic Constitutive Model of Miller and Walker by Numerical Experiments," International Journal of plasticity, Vol. 6, 1990, pp.189-206.

[58] Fatemi, A. and Yang, L., 1998, "Cumulative fatigue damage and life prediction theories: a survey of the state of the art for homogeneous materials," Int. J. Fatigue, 20, (1), pp. 9-34.

[59] Penny, R. K., 1996, “The use of Damage Concepts in Component Life Assessment," Int. J. Pres. Ves. \& Piping, 66, pp. 263-280.

[60] Qi, W. and Bertram, A., 1998, "Damage modeling of the single crystal superally SRR99 under monotonous creep," computational materials science, 13, (1), pp. 132-141.

[61] Haque, M. S. and Stewart, C. M., 2015, "Comparison of a new Sin-Hyperbolic Creep Damage Constitutive Model with The Classic Kachanov-Rabotnov Model Using Theoretical and Numerical Analysis," Proceedings of TMS 144th annual meeting and exhibition.

[62] Jablonski, D., 1978, "Fatigue Behavior of Hastelloy-X at elevated temperatures in air, vacumm and oxygen environments," $\mathrm{PhD}$ dissertation, Massachessetts Institute of Technology Department of Materials Science and Engineering.

[63] Haynes International, 1997, "Hastelloy X Alloy," Haynes International High-Temperature Alloys, H-3009C.

[64] Lee, S.Y., et al, 2008, "High-Temperature Tensile-Hold Crack-Growth Behavior of HASTELLOY X alloy compared to HAYNES 188 and HAYNES 230 alloys," Mechanics of Time-Dependent Materials, 12, (1), pp. 31-44. 
[65] Haynes International, "High Performance Alloys Technical Information: Hastelloy X Alloy,” [Online]. Available: http://www.haynesintl.com/pdf/h3009.pdf [Accessed: 4/6/2015]

[66] AK Steel, 304/304L Stainless Steel Specification Sheet. West Chester, OH.

[67] North American Stainless Specification Sheet. Carroll County, Kentucky.

[68] Stewart, C. M., 2013, "Mechanical Modell of a Gas Turbine Superalloy Subject to CreepFatigue," PhD dissertation, University of Central Florida Department of Mechanical and Aerospace Engineering.

[69] Corona, A., Marchesi, M., Martini, C., and Ridella, S., 1987, "Minimizing Multimodal Functions of Continuous Variables with the "Simulated Annealing" Algorithm," ACM Transactions on Mathematical Software, 13(3), pp. 262-280.

[70] Goffe, W. L., Ferrier, G. D. and Rogers, J., "Global Optimization of Statistical Functions With Simulated Annealing," May 1993.

[71] Frederick, C. O. and Armstrong, P. J., 2007, "A Mathematical representation of the multiaxial Bauschinger effect," Materials at High Temperatures, 24, (1). 


\section{APPENDIX}

\section{APPENDIX 1: HYBRID MODEL V2 UPF}

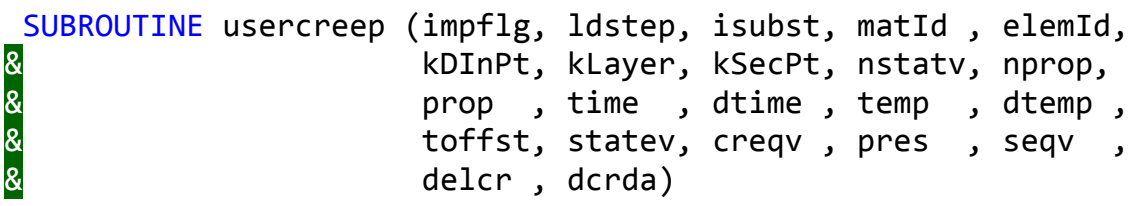

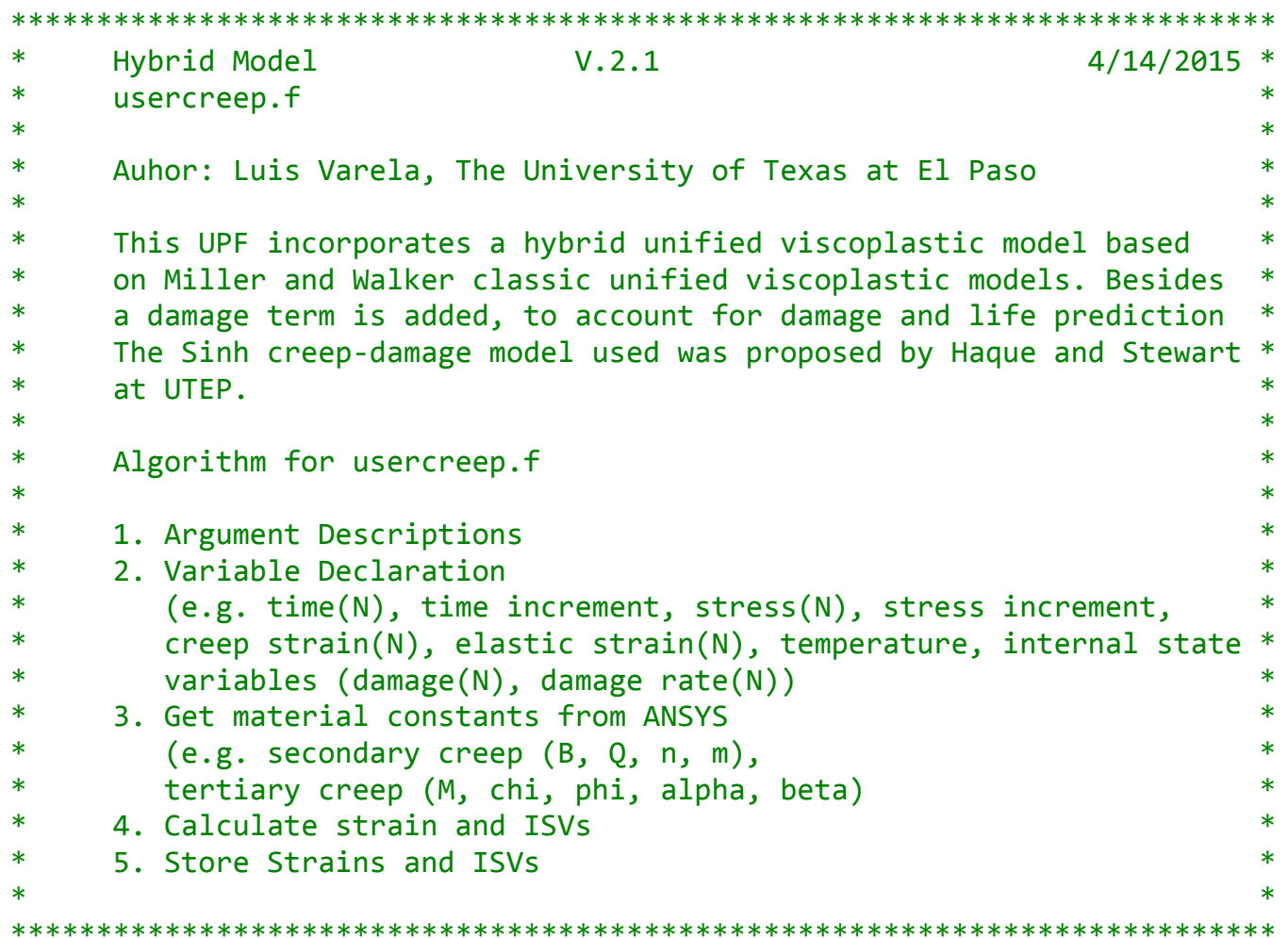

*2* VARIABLE DECLARATION

!\#include "impcom.inc"

DOUBLE PRECISION ZERO

PARAMETER $\quad($ ZERO $=0.0 \mathrm{~d} 0)$

!

! GLOBAL Arguments

! ===============

!

$\begin{array}{ll}\text { INTEGER } & \text { ldstep, isubst, matId, elemId, } \\ \text { kDInPt, kLayer, kSecPt, nstatv, } & \text { impflg, nprop, i, j } \\ \text { DOUBLE PRECISION dtime, time , temp , dtemp, toffst, }\end{array}$ 


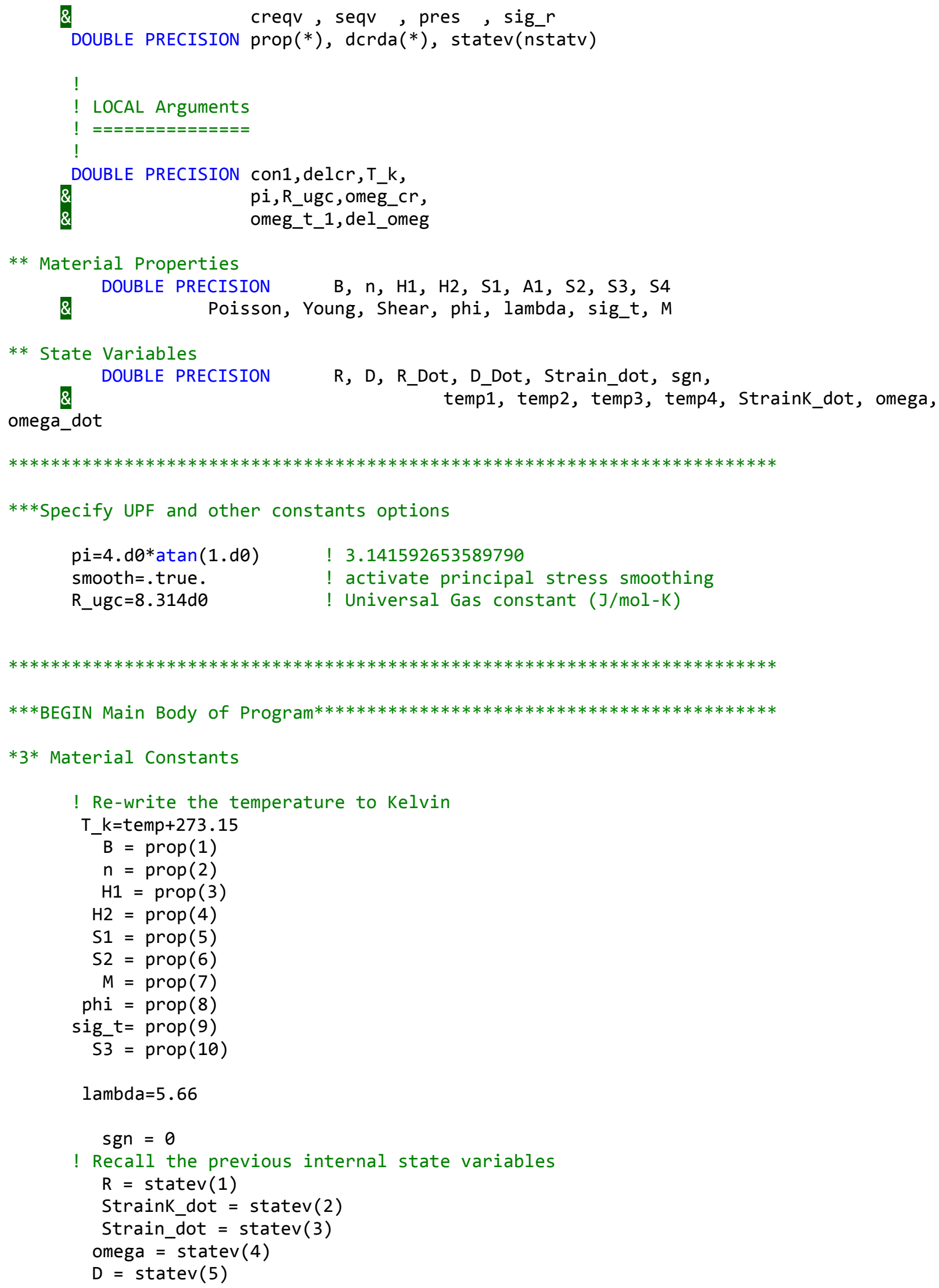


** REST STRESS RATE / KINEMATIC HARDENING

CALL NN(R, sgn)

R_dot $=H 1 * S t r a i n \_d o t-S 1 *(a b s(R)) * S t r a i n K \_d o t$

** DRAG STRESS RATE / ISOTROPIC HARDENING

temp1=abs (Strain_dot)

! $\mathrm{D}=\mathrm{H} 2$

D_dot $=\mathrm{H} 2 *$ Strain_dot-S2*dTime*(D-S3)**n

** DAMAGE RATE EQUATION

omega_dot $=(1 /$ phi $) * M *(1-\operatorname{EXP}(-$ phi $)) *((\operatorname{SINH}(($ seqv $) /$ sig_t $)) * * 3)$

\& *EXP(phi*omega)

** INELASTIC STRAIN RATE

If (D.eq. 0) then

Strain_dot $=0$

ELSE

temp1=seqv - R

CALL NN(temp1,sgn)

temp2 $=($ abs $($ temp1 $) / D) * * n$

Strain_dot=B*( $\sinh ($ temp 2$)) * \operatorname{sgn} * \exp ($ lambda*(omega**3/2))

ENDIF

! Determine the incremental creep strain

delcr=Strain_dot*dtime

! Determine derivative of incremental creep strain w.r.t. effective stress

CALL HH(temp1, temp3)

CALL HH(temp1-1, temp4)

temp $5=($ abs $($ temp1 $) / D)$

$\operatorname{dcrda}(1)=2 *($ temp3-temp4)*B*dtime* $(\sinh (($ temp5 $) * * n))$

\& *exp (lambda*(omega**3/2))

$+(B * d$ time*n*sgn*sgn*cosh (temp5)*exp (lambda*(omega**3/2))

\& $*(\sinh ($ temp5 $) * *(n-1))) / D$

! Determine derivative of incremental creep strain w.r.t. effective creep strain $\operatorname{dcrda}(2)=0$

*5* Store Strains and ISVs

$\mathrm{R}=\mathrm{R}+\mathrm{R} \_$dot*dTime

StrainK_dot=abs (Strain_dot)

omega=omega+omega_dot* $* \bar{d}$ Time

D=D+D_dot*dTime

statev $(1)=R$

statev(2) = Straink_dot

statev $(3)=$ Strain_dot 


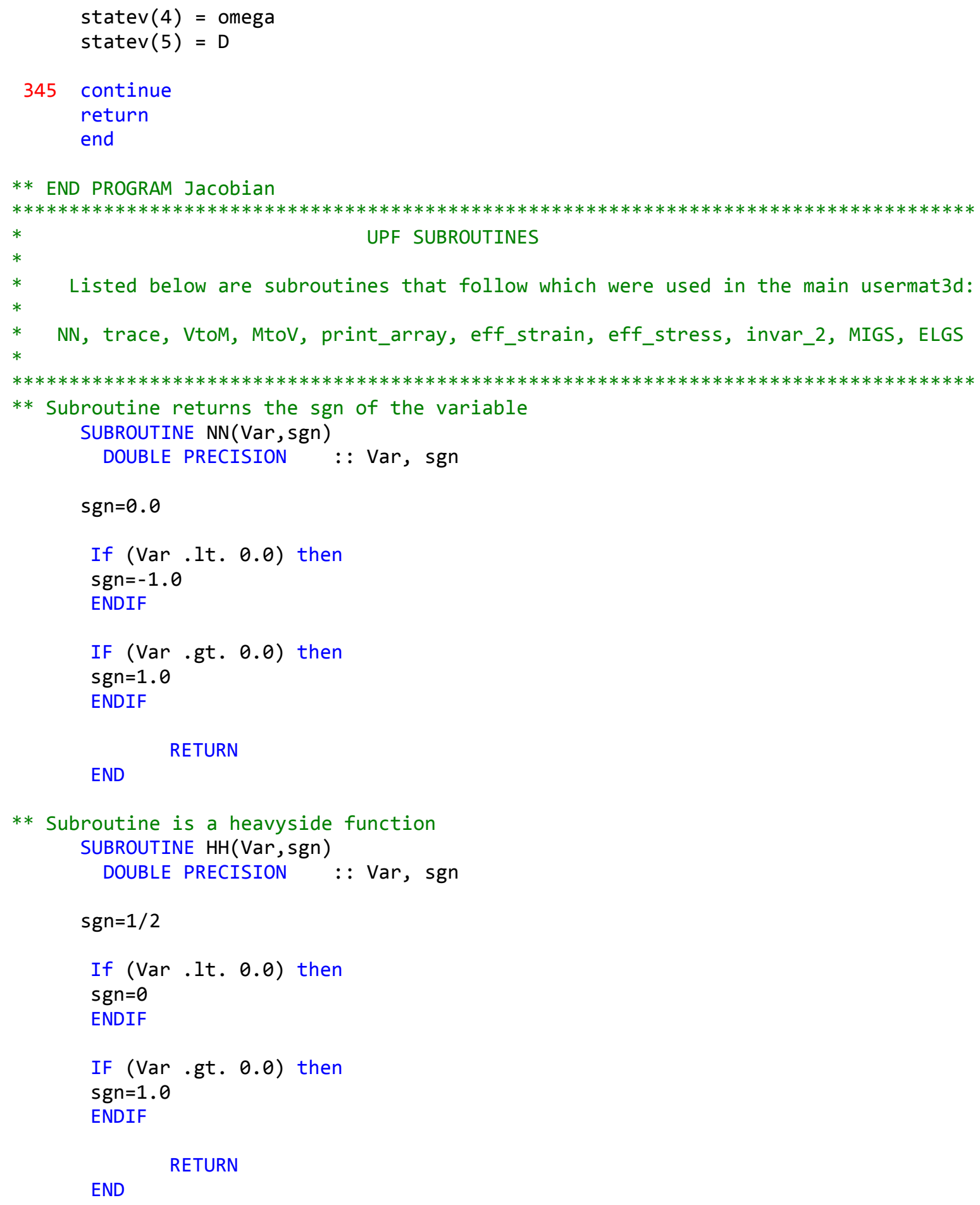




\section{VITA}

Luis Varela was born on September $24^{\text {th }}, 1990$ in Juarez, Chihuahua, Mexico. Luis graduated from the Preparatoria El Chamizal in Cd. Juarez in June 2008. Being enrolled in The University of Texas at El Paso he completed his undergraduate studies and received his Bachelor of Science in Mechanical Engineering in May 2013. In August 2013, Luis began his master's degree in Mechanical Engineering, and started to work as a teacher assistant and as a graduate research assistant under the supervision of Dr. Calvin M. Stewart. After graduation Luis would like to pursue a career in the Mechanical Engineering track in industry.

Permanent address: 3206 Rancho Parrita, Pradera Dorada

Cd. Juarez, Chihuahua, Mexico, 32618

This thesis was typed by Luis Alejandro Varela Jimenez. 